2

UCRL-MA-113603

in

CAVEMAN

Configuration And VErsion MANagement

User's Guide

v2.1.2

Friday, March 12, 1993

Kathleen. M Oyer

LLNL

AUG $02 \$ 0,3$

OSTI

an an an an an an an an en

MASTER

DISTRIBUTION OF THIS DOCUMENT IS UNLIMITED $g 7 D^{\circ}$ 


\section{DISCLAIMER}

This document was prepared as an account of work sponsored by an agency of the United States Government. Neither the United States Government nor the University of California nor any of their employees, makes any warranty, express or implied, or assumes any legal liability or responsibility for the accuracy, completeness or usefulness of any information, apparatus, product or process disclosed, or represents that its use would not infringe privately owned rights. Reference herein to any specific commercial products, process, or service by trade name, trademark, manufacturer, or otherwise, does not necessarily constitute or imply its endorsement, recommendation, or favoring by the United States Government or the University of California. The views and opinions of authors expressed herein do not necessarily state or reflect those of the United States Government thereof, and shall not be used for advertising or product endorsement purposes.

Work performed under the auspices of the U.S. Department of Energy by Lawrence Livermore National Laboratory under Contract W-74,05-Eng-48. 


\section{Table of Contents}

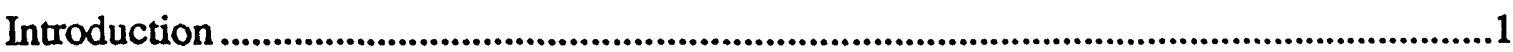

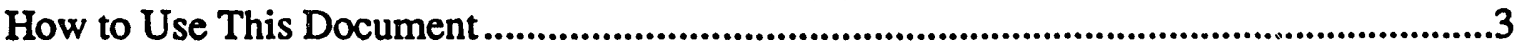

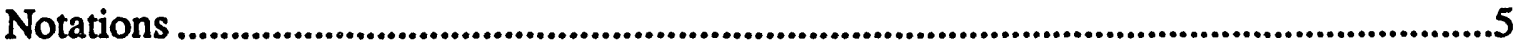

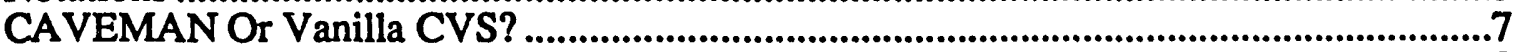

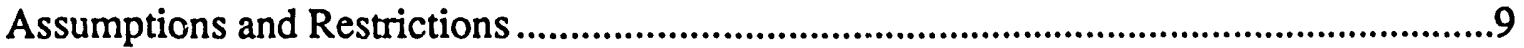

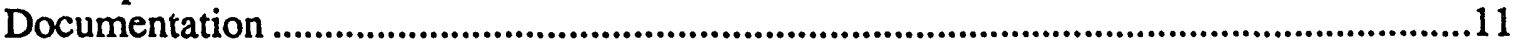

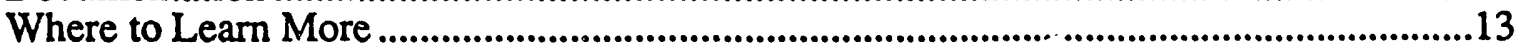

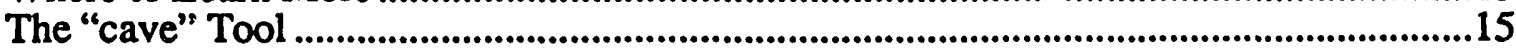

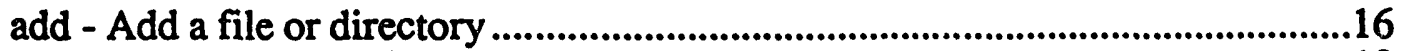

checkout - Check out files .....................................................................................18

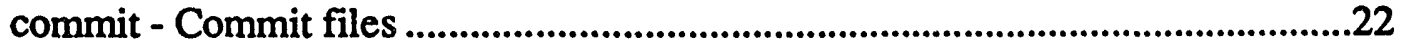

diff - Compare working files with repository .......................................................25

export - Check out files without CVS directories .................................................27

header - Add an rcs keyword to a file ............................................................30

help - Get information on how to use cave ...................................................32

history - Report on activities affecting a source repository .................................33

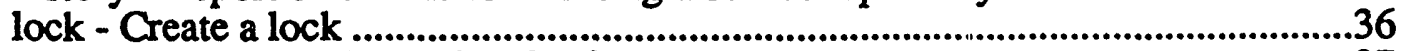

$\log$ - Display $\log$ information for files ................................................................37

pack - Collect files together for distribution ........................................................39

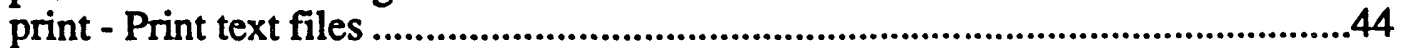

release - Cancel a check out .....................................................................48

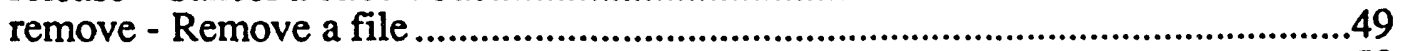

reserve - Reserve a file or directory ............................................................50

rtag - Assign symbolic tags to sources .........................................................52

status - Report on the current status of files ..................................................54

tag - Assign symbolic tags to sources ...........................................................55

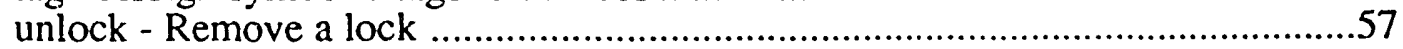

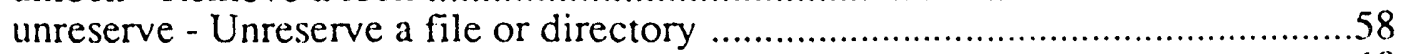

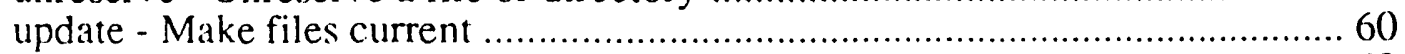

verify - Check the project description file .....................................................63

version - Report cave's version number .....................................................64

what - Get information about a project .........................................................65

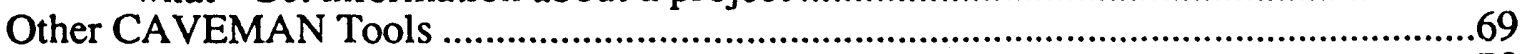

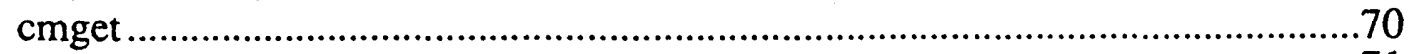

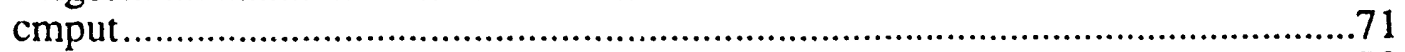

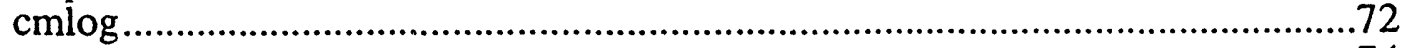

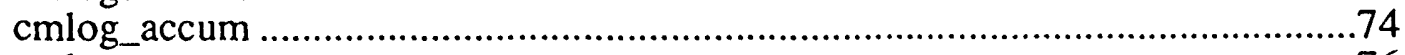

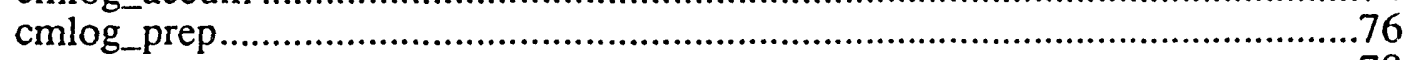

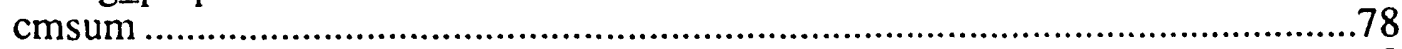

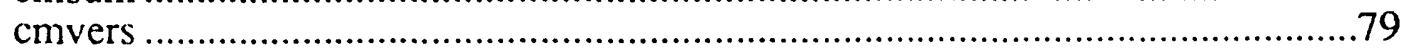

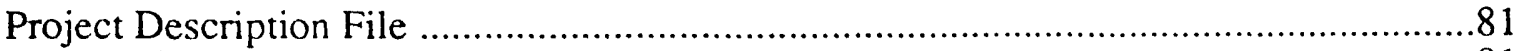

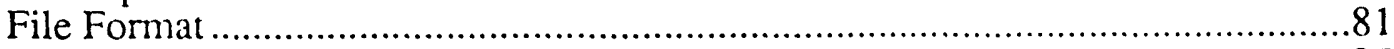

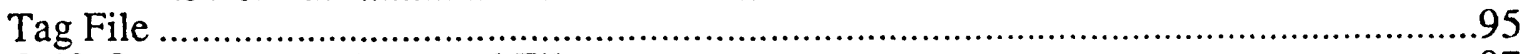

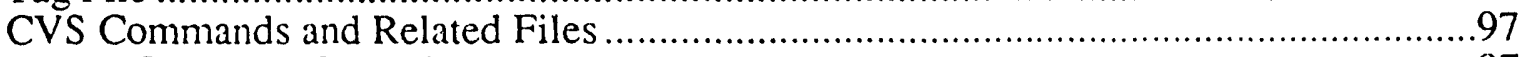

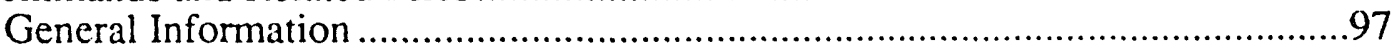




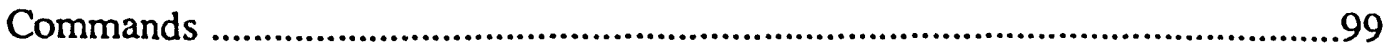

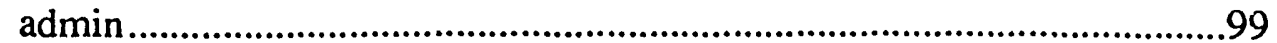

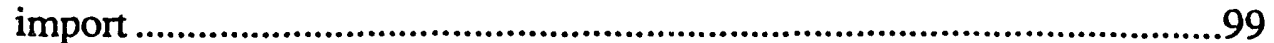

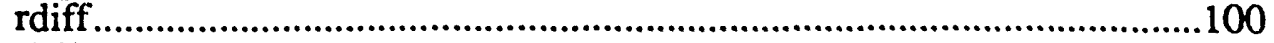

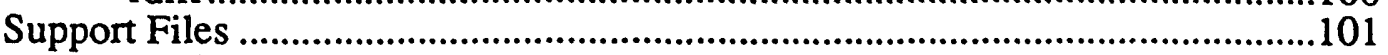

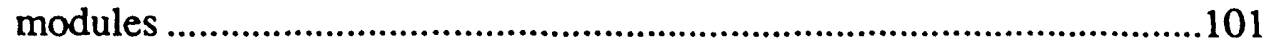

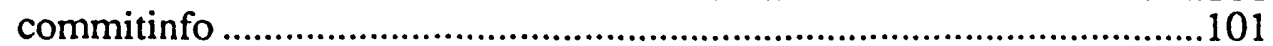

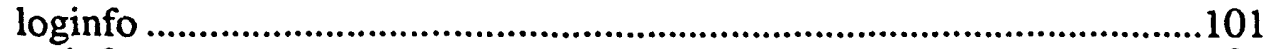

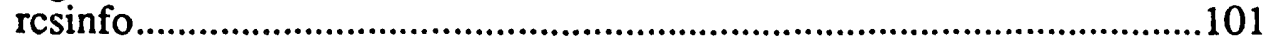

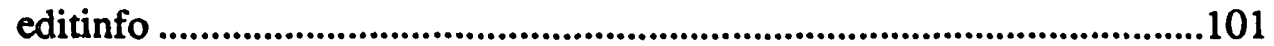

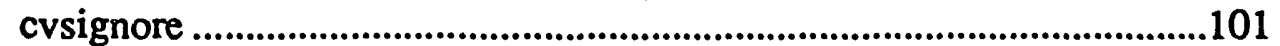

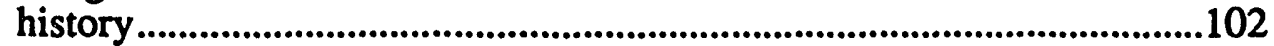

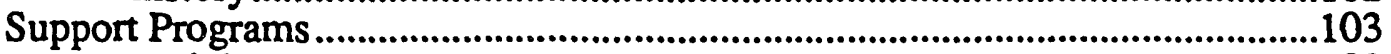

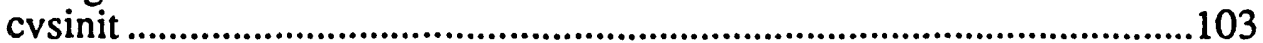

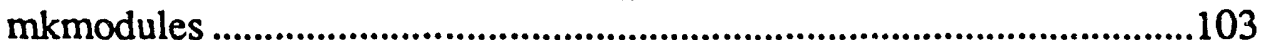

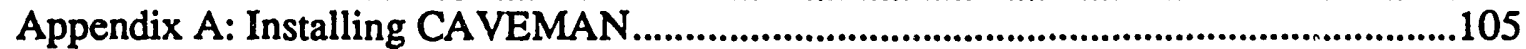

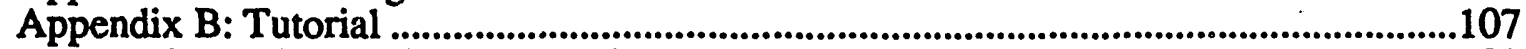

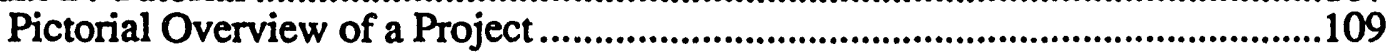

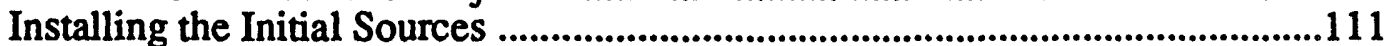

Adding a Second Root Directory to a Project .....................................................117

Adding a Repository to an Existing Root Directory ........................................123

Retrieving Files From a Repository ................................................................127

Adding a Directory to a Repository .............................................................133

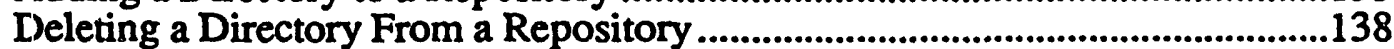

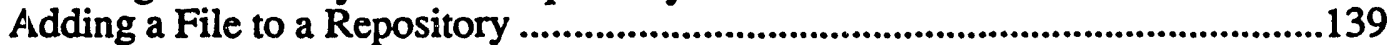

Deleting a File From a Repository ................................................................143

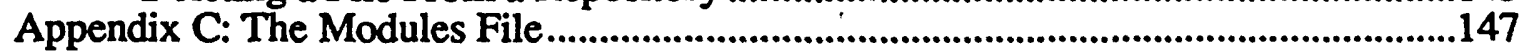

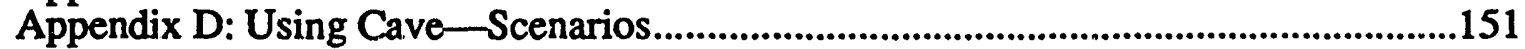

Sources and executables on one workstation...............................................151

Sources on a workstation and executables on another platform. .....................152

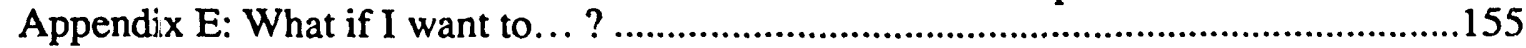

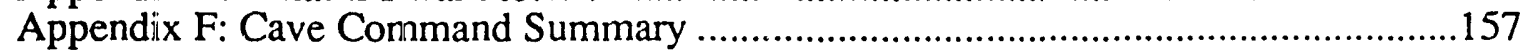

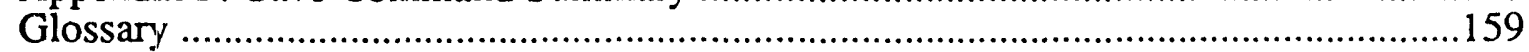

Index 


\section{Introduction}

The mission of the CAVEMAN project is to provide the tools for large code systems so that those who work on them can perform source control and configuration control on UNIX-based file servers, rather than the LC Crays.

An outside product called cvs has been chosen as the underlying source control system. The initials stand for "Concurrent Versions System". It is a freeware program that runs on most UNIX stations. To quote from the cvs man page:

"cvs is a front end to the res revision control system which extends the notion of revision control from a collection of files in a single directory to a hierarchical collection of directories consisting of revision controlled files. These directories and files can be combined together to form a software release. cvs provides the functions necessary to manage these software releases and to control the concurrent editing of source files among multiple software developers."

While cvs may be run directly, there are several tasks in our environment that it seemed either safer or easier to automate. CAVEMAN provides the needed functionality, generally in the form of wrappers to cvs. 


\section{How to Use This Document}

If you are new to CAVEMAN and cvs read the chapters or sections (they are all short):

Table of Contents (to see what reference materials are available in the document).

Notations

CAVEMAN Or Vanilla CVS?

Assumptions and Restrictions

Documentation

Where to Learn More

Glossary

Then look over "Appendix B: Tutorial", and "Appendix E: What if I want to... ?".

Experienced CAVEMAN users should use this document as a reference. The most useful chapters are:

The "cave" Tool

Other CAVEMAN Tools

Project Description File

Tag File

CVS Commands and Related Files

Appendix C: The Modules File

Appendix F: Cave Command Summary 
How to Use This Document 


\section{Notations}

The following conventions are used in this document:

bold

italic

[]

I

$\cdots$
Literal values; commands, parameters, options, file names, directory names.

Variable for which you supply values.

In syntax lines, indicate optional items.

Separates mutually exclusive commands or options.

You can repeat the preceding argument, delimiting the arguments by spaces. 


\section{CAVEMAN Or Vanilla CVS?}

Although CAVEMAN and cvs look quite similar to the casual user, there are some significant differences.

It is very important to note that CAVEMAN is built entirely as a shell on top of cvs. There are no hacks to the cvs source code. In most cases, one can switch back and forth between CAVEMAN and cvs, if it is necessary.

\section{CAVEMAN Advantages:}

- Introduces the concept of a project, where sources may be spread among more than one root directory.

- Automatically forces any checked out files into a local directory structure which echoes that of the root directories. Any additional scripts you may need are a great deal easier to write, because the local files are in predictable locations.

- Allows additional locks to be set during some operations, ensuring that the repositories don't change unexpectedly.

- Scripts to make the log méssages look nice.

- Additional hooks for user specified programs to be run before and after checkout and commit.

- Automatic tagging of all files in project when anything is committed.

- The what command to provide information about a project, such as who has access and what files are involved.

- The pack command, to collect files together for distribution to another machine.

- The print command, to send text files to a printer.

- A built in help package.

- Additional synonyms for several commands.

- A (mostly) order-independent execute line.

- More defaults on the execute line.

- Most commands have an option which allows the list of files to be processed to be read in from a command file.

- More error checking.

CAVEMAN Disadvantages:

- All the usual disadvantages of layered programs.

- More levels of directories in the local workspace. 
- The way CAVEMAN enforces the directory structure causes some of the commands to require pathnames be given relative to a special project directory, not relative to what directory the user is actually in. This can be very confusing for beginners, and is not like normal UNIX. (If there is such a thing as normal UNIX.)

- The way CAVEMAN enforces the directory structure causes some of the fancier features of the modules file not to behave in the same manner as they do with plain cvs.

- Because of the enforced directory structure, there are some restrictions on names for the various components.

- Some of the commands are slightly different than the crs equivalents. This may be confusing. 


\title{
Assumptions and Restrictions
}

\author{
CAVEMAN is installed and maintained at LLNL: \\ Closed Labnet $\quad$ Peregrine (130.106.66.1), an A Division machine. \\ Open Labnet Contact the author.
}

Certain assumptions need to be made, and some restrictions enforced, in order to provide the automation that is required. (The terms used in this discussion are defined in the Glossary near the end of the document.)

The main tool in CAVEMAN is a perl script named cave. It acts as a front end to cvs, and provides several other capabilities as well.

A project is the set of files and directories under cave control which are used to create a given program. They may include source files, documents, make files, scripts, etc.

A project may use one or more root directories. The project may use one or more source repositories within each root directory.

The functions within cave are customized for an individual project through the use of a project description file. This is a text file which describes the project's root directories, source repositories, access rights, locks, and other information specific to a particular project. The path to the project description file may be set with the environment variable CAVE_PD.

The inodules file is a special file used by cvs for administrative purposes. It is used to define alias's, and to define keys or pointers to the source repository, its subdirectories, and its source files. It is assumed that any modules file used by the project is set up so that a request to check out a source repository results in the entire source repository being checked out. It is also assumed that the modules file contains the standard CVSROOT keys:

\# CVSROOT support
CVSROOT
modules
loginfo
commitinfo
rcsinfo
editinfo
cVsignore

-i /usr/local/bin/mkmodules CVSROOT
$-i$ /usr/local/bin/mkmodules CVSROOT modules
- $i$ /usr/local/bin/mkmodules CVSROOT loginfo
-i /usr/local/bin/mkmodules CVSROOT commitinfo
- $i$ /usr/local/bin/mkmodules CVSROOT rCsinfo
- $i$ /usr/local/bin/mkmodules CVSROOT editinfo
$-i$ /usr/local/bin/mkmodules CVSROOT cVsignore

CAVEMAN forces any checked out files into a local directory structure which echoes that of the root directory. This is absolutely necessary, to ensure that later requests to 
pack or commit the files result in the proper actions. This behavior is different than cvs, which does not enforce any sort of local directory structure.

The way CAVEMAN enforces the directory structure causes some of the fancier features of the modules file (such as the $-\mathbf{i}, \mathbf{- o}$ and $-t$ options) not to behave in the same manner as they do with plain cvs. For more information on restrictions and how to set up a modules file for CAVEMAN, see "Appendix C: The Modules File".

Because of the enforced directory structure, there are some restrictions on names for the various components. (In this context, "name" refers to the tail of the absolute pathname.) 'The name of the project should be unique. There should be no root directory, source repository, directory or file used by the project which has the same name as the project. Also, all root directory and source repository names used by a project should be unique.

There is an issue to consider when adding subdirectories to an existing source repository. When a user requests that cvs/cave add a subdirectory, it immediately adds an empty subdirectory at the correct place in the source repository, and creates a directory called CVS inside the local copy of the subdirectory. Most other commands to cvs/cave do not affect the source repository directly until a commit is done. The new, empty subdirectory in the source repository does not become populated until the commit. Some people may choose to wait until just before the commit process to add the subdirectory, so that the source repository doesn't get affected too early. There is a possible problem, however. The CVS directory and its contents are used by several commands in the CAVEMAN system to automate information handling. The CVS directory is created for a new directory by running cvs/cave add. Because of this, some may prefer to add the subdirectory immediately. 


\section{Documentation}

This document, the CAVEMAN User's Guide, provides the most complete documentation for the CAVEMAN system. Copies are available from the author:

Kathleen Dyer

x3-6803

L-16

A PostScript version of the document is also available with the CAVEMAN distribution.

The main tool for CAVEMAN is a perl script named cave. It has a help package. To access it, type:

cave help I more

The tools which are part of the caveman system have man pages. Use them as a quick reference.

The programs used by CAVEMAN (cvs, rcs, diff3, and perl) have man pages. Read them for more thorough descriptions of the programs. Also, a book about perl is available from O'Reilly \& Associates-Programming perl.

Many of the descriptions for cave commands are borrowed in whole or in part from the man page descriptions of the related cvs and rcs commands. Much of the discussion of cvs is copied from its man page. 
Documentation

:

;

Caveman User's Guide

12

March 12, 1993 


\section{Where to Learn More}

In addition to all the documentation, there is a very active cvs mailing list on Internet. To have your name added to the list, send an email message to:

info-cvs-request@prep.ai.mit.edu

To ask questions, report bugs or post cvs related information, send an email message to:

info-cvs@prep.ai.mit.edu 


\section{The "cave" Tool}

While cvs may be run directly, there are several necessary tasks that it seems either safer or easier to automate. The CAVEMAN project provides tools to help with source control and configuration management.

The main tool is a perl script named cave. It acts as a front end to cvs, and provides several other capabilities as well. The execute line for cave is:

cave [-d proj_desc] [....] [-L] [cave_command [command_options]]

-d proj_desc

$-\mathbf{L}$

cave_command

command_options
Name of the project description file used to describe the source control and configuration parameters for a project. If more than one - $d$ command is entered, only the last one is used. This overrides the setting of the CAVE_PD environment variable.

Ignore any locks (except for a panis lock) which are normally checked for and which may be set for the project. A message is sent to ne standard output if any locks are present. A mail message is sent to anyone who has a lock which is ignored. The default for a cave_command which reads from or writes to a repository is to stop if locks are present.

Select the required functionality. The default is "verify". The commands are described on the following pages.

Each cave_command has a set of options which are described on the following pages.

cave reads information from the project description file into memory, and uses that information to set values needed by the different commands. Some commands do not require the project description file and do not read it, but it is not an error to include its name on the command line.

The project description file is described in the chapter "Project Description File".

As much as possible, the options for each command match those used for the related cvs command. 


\section{add - Add a file or directory}

This command to cave is used to add new files or directories to a source repository. add [-R] [-k kflag] [-m message] [-F list] file ...

Synonym: new

$-\mathbf{R}$

-k kflag

-m message

-F list

A flag to recursively descend into any directory given in the file list, and add its contents. The default is to not descend. You may not add a directory which is already known to cvs.

Specifies the default way that this file will be checked out. The kflag option is stored in the source file and can be changed with cvs admin. The possible values of kflag are described in the $\operatorname{rcs}(1)$ man page.

A quoted string containing a message to be included with the file(s). The message is stored in the file(s) when it is committed. It is considered "descriptive text" by rcs, and may be viewed by, typing "cave log - $t$ file".

list is a file containing the names of files to be used with this command.

Each name must be in the same form as file.

Blank lines and leading blanks are allowed.

A "\#" signals the start of a comment.

Multiple lines are allowed. More than one entry may be on a line. If so, they must be blank delimited.

The -F option may be used in addition to or in place of file.

file

The name of a file or directory which already exists in the current local directory. Specifying a directory without using the $-\mathbf{R}$ option causes the directory to be added, but not its contents.

The files to add must exist in the current local directory, which must have been created by cave checkout or cvs checkout (it contains a CVS directory). The files are not placed in the source repository immediately. Rather, they are marked as new files and only placed in the source repository when a commit is done.

If file is actually the name of a directory, the process is a little different. The directory is immediately created at the correct place in the source repository, but it remains empty. The corresponding directory in the local workspace gains a CVS directory. You must then go into the local directory, and add the files. If the -R option is used, this is done automatically. These files are marked as new files, and placed in the source repository when a commit is done. 
Use add to undo a remove command, unless the change has already been committed.

Returns:

\begin{tabular}{|l|l|c|}
\cline { 2 - 3 } \multicolumn{1}{c|}{} & Standard Out/Error & exit value \\
\hline Success & Message & 0 \\
\hline Error & Error message. & 1 \\
\hline
\end{tabular}

The project description file is required when cave runs this command. 


\section{checkout - Check out files}

This command to cave is used to check out source files from a cvs repository.

checkout [cvs_checkout_opts] [-res] [-F list] [path ...]

Synonym: co, get, out

-res

- F list

path
Reserve each directory or file as it is checked out. See the cave reserve command for a description of what it means to reserve something. If there is a conflict between something you wish to reserve and a previous reservation, the directory or file is still checked out but a warning message is sent stating that it could not be reserved.

list is a file containing the names of paths to be used with this command.

Each name must be in the same form as path.

Blank lines and leading blanks are allowed.

A "\#" signals the start of a comment.

Multiple lines are allowed. More than one entry may be on a line. If so, they must be blank delimited.

The -F option may be used in addition to or in place of path.

A pathname. In the simplest case, when a project only uses one root directory, it must be one of the following:

- A key, as defined in the modules file for this root directory.

\section{module}

A pathname which must start with the tail of a source repository name. It may continue, to include the name of a subdirectory or file.

$$
\text { repository[/dir[/...]] }
$$

If a project uses more than one root directory, the pathname must start with the tail of the absolute root directory pathname. It may continue with:

- $\quad$ A key, as defined in the modules file for this root directory.

$$
\text { root_dirlimodule] }
$$

- The relative pathname of a source repository and its contents. 


\section{root_dir[/repository[/dir[/...]]]}

If path ends with a source repository or subdirectory name, all files contained within that source repository or directory are included (unless the -1 option was used).

The files must exist in the source repository, and be under cave control. Do not include the ", $v$ " suffix when specifying file names. The default is to check out all the files which are under cave control and are a part of the project.

The most common cvs_checkout_opts are:

-D date

$-\mathbf{f}$

$-1$

$-\mathbf{P}$

$-\mathbf{p}$

$-Q$

$-\mathbf{q}$

-r tag
Use the revision that was checked in at or before the specified date argument. The date is a single gument, date description specifying a date in the past. The formats are similar to those described in co(1). Examples of valid date specifications include:

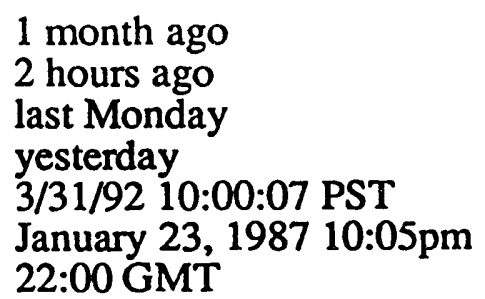

1 month ago

2 hours ago

last Monday

yesterday

3/31/92 10:00:07 PST

January 23, 1987 10:05pm

22:00 GMT

Forces the specified tag to match. Normally, if a tag is specified and the requested file does not contain the tag, the file is ignored. The -f option causes any file which does not contain the tag to be retrieved even when there is no match for the tag or date.

Local. Do not recursively descend the directory structure.

Prune directories that are empty. This does not remove the directory from the repository, only from your checked out copy.

Pipe the files to standard output, rather than writing them to the local directory.

Causes the command to be really quiet.

Causes the command to be somewhat quiet.

Use the revision of the file which was marked with the symbolic tag "tag". To avoid getting empty directories, combine this option with the -P option:

$$
\text { -P -r tag }
$$

The default is to check out the latest version. 
The more advanced cvs_checkout_opts are:

$-\mathbf{A}$

$-\mathrm{j}$ branch

-k kflag

$-\mathbf{n}$

$-S$
Reset any sticky tags, dates or -k options. (If you get a working file using one of the $-r,-D$, or $-k$ options, cvs remembers the corresponding tag, date or kflag and continues using it on future updates. Use the -A option to make cvs forget these specifications.)

Merges the changes between two different branches of development. For more information, read the cvs man pages.

Alter the default processing of res keywords; all the - $k$ options described in rcs(1) are available. Your kflag specification is "sticky" when you use it to create a private copy of a source file. That is, when you use this option with the checkout or update commands, cvs associates your selected kflag with the file, and continues to use it with future update commands on the same file until you specify otherwise. The kflag option is stored in the source file and can be changed with cvs admin.

Causes the command not to run any checkout program as specified in the modules file.

Display per-module status information within the modules file.

Returns:

\begin{tabular}{|l|l|c|}
\cline { 2 - 3 } \multicolumn{1}{c|}{} & Standard Out/Error & exit value \\
\hline Success & Message & 0 \\
\hline Error & Error message. & 1 \\
\hline
\end{tabular}

The project description file is required when cave runs this command.

The checked out source files are placed in a directory named after the project, as determined from the project description file. They are placed so that the structure echoes that of the root directory.

Note: Checking out a file into a directory where it already exists has the same effect as running cave update.

The checkout process involves the following steps:

- Set an extract lock.

- Perform error checking of the command line.

- See if any of the directories and/or files to be checked out were reserved by someone else. Stop if there are any conflicts. 
- Run any programs as specified by any precheckout lines in the project description file. Any program which exits with a non-zero status is assumed to have failed, and cave ends with an error exit.

- Run cvs to checkout the files.

- Run any programs as specified by any postcheckout lines in the project description file. Any program which exits with a non-zero status is assumed to have failed, and cave ends with an error exit.

- Release the lock.

Each root directory is assumed to contain the cvs administrative directory called CVSROOT. This directory and its files may be checked out, but it must be explicitly requested in path.:

\begin{tabular}{|l|l|l|}
\cline { 2 - 3 } \multicolumn{1}{l|}{} & One root directory & More than one root directory \\
\hline $\begin{array}{l}\text { Path to use with } \\
\text { checkout }\end{array}$ & CVSROOT & $\begin{array}{l}\text { root_dir/CVSROOT } \\
\text { root dir/modules }\end{array}$ \\
\hline $\begin{array}{l}\text { File/directory created in } \\
\text { local project directory }\end{array}$ & $\begin{array}{l}\text { CVSROOT } \\
\text { CVSROOT/modules }\end{array}$ & $\begin{array}{l}\text { CVSROOT.root_dir } \\
\text { CVSROOT.root dir/modules }\end{array}$ \\
\hline
\end{tabular}

See the export command for another way to get files from a repository. Do not export and checkout files into the same directory. 


\section{commit - Commit files}

This command to cave is used to commit source files to cvs root directories.

commit [-InR] [-m msg I-f file] [-rrev] [-t tag] [-T] [-unres] [-F list] [path ...]

: Synonym: ci, in, put

$-1$

$-\mathbf{n}$

$-\mathbf{R}$

$-\mathbf{m} m s g$

-f file

$-\mathbf{r r e v}$

$-\mathrm{t} \operatorname{tag}$
Local. Do not recursively descend the directory structure. The default is to descend the directory structure.

Causes the command not to run any commit program as specified in the modules file.

Recursively descend into any subdirectories and commit any changes. This is the default.

A quoted string containing a message to be included in the $\log$ file for this commit. If neither the $-m$ or $-f$ options are used, an editor is invoked to allow you to enter a $\log$ message.

The name of a file containing a message to be included in the $\log$ file for this commit. If neither the $-\mathbf{m}$ or $\mathbf{- f}$ options are used, an editor is invoked to allow you to enter a log message.

Used to bring files up to a particular numeric revision. This affects any file specified in path which was checked out but not modified. (There is no space after the $-r$.)

These two options work together to determine which files are labeled with a tag, and the value of the tag.

-t tag

Set the value of the tag to tag. It must not contain a semicolon, colon, dollar sign, at sign, comma or period (;:\$@,.). Determine the files to be tagged from path.

-T Use the tag line in the project description file to determine the name of a tag file. Get tag from this file, and increment it by one. Determine the files to be tagged from the tag line in the project description file. Put the incremented tag back into the tag file.

$-1 \operatorname{tag}-\mathrm{T} \quad$ Set the value of the tag to tag. It must not contain a semicolon, colon, dollar 
-unres

-F list

path sign, at sign, comma or period (;:\$@,.). Determine the files to be tagged irom the tag line in the project description file. Put the new tag back into the tag file.

Unreserve each directory or file as it is committed. See the cave unreserve command for a description of what it means to unreserve something. If there is a conflict between something you wish to unreserve and one of your own previous reservations, the directory or file is still committed but a warning message is sent stating that it could not be unreserved.

list is a file containing the names of paths to be used with this command.

Each name must be in the same form as path.

Blank lines and leading blanks are allowed.

A "\#" signals the start of a comment.

Multiple lines are allowed. More than one entry may be on a line. If so, they must be blank delimited.

The -F option may be used in addition to or in place of path.

Pathname, relative to the project firectory, of a file or subdirectory to include in the commit. It must be under cave control in the project directory in your workspace. If path ends with a subdirectory name, all files contained within that directory and its s: bdirectories are included. Only files which have been modified are committed. If a file is specified in path and it is unchanged, cvs ignores it. The default is to commit all the files which are under cave control and have been changed in project.

Returns:

\begin{tabular}{|l|l|c|}
\cline { 2 - 3 } \multicolumn{1}{c|}{} & Standard Out/Error & exit value \\
\hline Success & Message & 0 \\
\hline Error & Error message. & 1 \\
\hline
\end{tabular}

The project description file is required when cave runs t'iis command.

The commit jrocess involves the following steps:

- Set an insert lock.

- Perform error checking of the command line.

- See if any of the directories and/or files to be committed were reserved by someone else. Stop if there are any conflicts. 
- Run cvs commit with the -n option. This causes cvs to attempt to execute the commit, but only to issue reports. It does not actually commit any files. This is done by cave as a final error check. It will catch problems, such as the case where a file you are trying to commit has been modified in the repository since you checked it out.

- Run any programs as specified by any precommit lines in the project description file. Any program which exits with a non-zero status is assumed to have failed, and cave ends with an error exit.

- Run cvs to commit the files.

- Tag files, as determined by the tag line in the project description file, and the -t and -T options.

- Run any programs as specified by any postcommit lines in the project description file. Any program which exits with a non-zero status is assumed to have failed, and cave ends with an error exit.

- If there is a mail line in the project description file, it is read to get a list of mail names. Everyone on the list is notified of the commit, and path is listed. If you would like to have a more complete message sent, consider using the -u option in cmlog or cmlog_accum instead. This causes a copy of the logfile message to be sent to anyone listed after the $-\mathbf{u}$.

- See if any of the directories and/or files that were committed are to be unreserved. Attempt to do so.

- Release the lock.

Each root directory is assumed to contain the cvs administrative directory called CVSROOT. If this directory and its files were checked out with cave, they were placed in a directory named CVSROOT (if there is only one root directory in the project) or CVSROOT.root dir (if there is more than one root directory in the project). This directory may be committed, but it must be explicitly requested in path. 


\section{diff - Compare working files with repository}

This command to cave is a simple interface to the cvs diff command. It is used to compare working files with revisions in the source repository.

diff [-1] [rcsdiff_options] [[-r rev1 | -D date1 ] [-r rev2 |-D date2 ]] [-F list] [file ...]

$-1$

$-\mathbf{r}$ rev

$-\mathrm{D}$ date

-F list

file
Local. Do not recursively descend the directory structure. The default is to descend the directory structure.

File revision to diff.

Use the revision that was checked in at or before the specified date argument. The date is a single argument, date description specifying a date in the past. The formats are similar to those described in co(1). Examples of valid date specifications include:

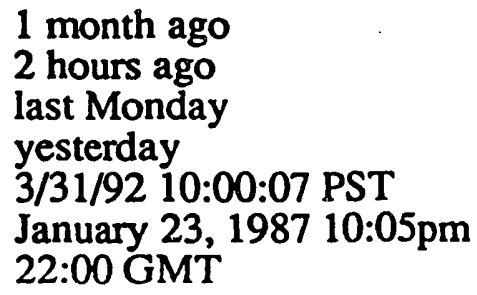

list is a file containing the names of files to be used with this command.

Each name must be in the same form as file.

Blank lines and leading blanks are allowed.

A "\#" signals the start of a comment.

Multiple lines are allowed. More than one entry may be on a line. If so, they must be blank delimited.

The - F option may be used in addition to or in place of file.

The name of a file to diff. The default is to report on all files and directories in the current local workspace.

The rcsdiff_options, which are passed to rcsdiff, are:

-ksubst

$-q$

$-V_{n}$
Affects keyword substitution when extracting revisions, as described in co(1). For example, "-kk -rl.1 -r1.2" ignores differences in keyword values when comparing revisions 1.1 and 1.2 .

Quiet mode. Reduces the number of messages to standard out.

Emulate RCS version $n$. See co(1) for details. 
-xsuffixes

diff_options
Use suffixes to characterize RCS files. See ci(1) for details.

These options are passed to diff. They may vary, depending on the version of diff on your system.

If you don't specify a particular revision using the $-\mathbf{r}$ or $\mathbf{- D}$ options, your files are compared with the revisions they were based on. If two revisions are specified, you can see the differences between two revisions in the repository. The $-r$ and $-D$ commands may be mixed together, with at most two options ever specified.

This command must be called from within or below the project directory.

See the man pages cvs, rcsdiff and diff for more detailed explanations of the options.

Returns:

\begin{tabular}{|l|l|c|}
\cline { 2 - 3 } \multicolumn{1}{c|}{ Standard Out/Error } & exit value \\
\hline Success & Report. & 0 \\
\hline Error & Error message. & 1 \\
\hline
\end{tabular}

The project description file is required when cave runs this command. 


\section{export - Check out files without CVS directories}

This command to cave is used to check out source files from a cvs repository. It differs from checkout in that no CVS administrative directories are created.

$$
\text { export [-fnQq] -r tag I-D date [-F list] [module ...] }
$$

Synonym: read

$-\mathbf{f}$

$-\mathbf{n}$

$-\mathbf{Q}$

$-\mathbf{q}$

$-r$ tag

$-\mathrm{D}$ date
Forces the specified tag to match. Normally, if a tag is specified and the requested file does not contain the tag, the file is ignored. The -f option causes any file which does not contain the tag to be retrieved even when there is no match for the tag or date.

Causes the command not to run, iy checkout program as specified in the modules file.

Causes the command to be really quiet.

Causes the command to be somewhat quiet.

Use the revision of the file which was marked with the symbolic tag "tag".

Use the revision that was checked in at or before the specified date argument. The date is a single argument, date description specifying a date in the past. The formats are similar to those described in co(1). Examples of valid date specifications include:

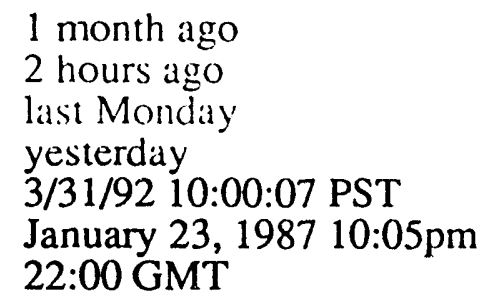

-F list

list is a file containing the names of modules to be used with this command.

Each name must be in the same form as module.

Blank lines and leading blanks are allowed.

A "\#" signals the start of a comment.

Multiple lines are allowed. More than one entry may be on a line. If so, they must be blank delimited.

The -F option may be used in addition to or in place of module. 
module
A key, as defined in the modules file for this root directory. If a project uses more than one root directory, module must start with the tail of the absolute root directory pathname and continue with the module, as defined in the modules file:

$$
\text { root_dir/module }
$$

The default is to use all the repository names defined for this project. If they are defined correctly in the modules file, this causes all the files under cave control to be exported.

Returns:

\begin{tabular}{|l|l|c|}
\cline { 2 - 3 } \multicolumn{1}{c|}{ Standard Out/Error } & exit value \\
\hline Success & Message & 0 \\
\hline Error & Error message. & 1 \\
\hline
\end{tabular}

The project description file is required when cave runs this command.

This command requires that you specify a date or tag (with $-D$ or $-\mathbf{r}$ ).

The -kv option is always set when export is used. This causes any res keywords to be expanded such that an import done at some other site will not lose the keyword revision information. There is no way to override this.

The exported source files are placed in a directory named after the project, as determined from the project description file. They are placed so that the structure echoes that of the root directory. Since no CVS directories are created, these copies of the files cannot be committed back into the repository.

Each root directory is assumed to contain the cvs administrative directory called CVSROOT. This directory and its files may be exported, but it must be explicitly requested in path.:

\begin{tabular}{|c|c|c|}
\hline & One root directory & More than one root directory \\
\hline Path to use with export & $\begin{array}{l}\text { CVSROOT } \\
\text { modules } \\
\end{array}$ & $\begin{array}{l}\text { root_dir/CVSROOT } \\
\text { root dir/modules }\end{array}$ \\
\hline $\begin{array}{l}\text { File/directory created in } \\
\text { local project directory }\end{array}$ & $\begin{array}{l}\text { CVSROOT } \\
\text { CVSROOT/modules }\end{array}$ & $\begin{array}{l}\text { CVSROOT.root_dir } \\
\text { CVSROOT.root dir/modules }\end{array}$ \\
\hline
\end{tabular}

See the checkout command for another way to get files from a repository. 
Note: Do not export and checkout files into the same directory. 


\section{header - Add an rcs keyword to a file}

This command to cave is used to add a comment line containing an rcs keyword to the top of an ASCII file in a local directory.

header [-q] [-c chars] [-k keyword [-k keyword ...]] [-F list] file [...]

$-\mathbf{q}$

-c chars

-k keyword

-F list
Quiet mode. Reduces the number of messages to standard out.

Use chars as the first entry in the comment line. The default is to use "c". If chars contains a meta-character that may be interpreted by the shell, such as *, it should be protected with single quotes.

A keyword to add at the top of the file. The keyword is automatically bracketed by dollar signs (\$). The default is to use "Id".

list is a file containing the names of files to be used with this command.

Each name must be in the same form as file.

Blank lines and leading blanks are allowed.

A "\#" signals the start of a comment.

Multiple lines are allowed. More than one entry may be on a line. If so, they must be blank delimited.

The -F option may be used in addition to or in place of file.

file

The keyword \$Id $\$$ is used by cvs and rcs to place modification information. Other keywords are Author, Date, Header, Locker, Log, RCSfile, Revision, Source and State. They are described in the man page co(1).

Returns:

\begin{tabular}{|l|l|c|}
\cline { 2 - 3 } \multicolumn{1}{c|}{} & Standard Out/Error & exit value \\
\hline Success & A series of status messages. & 0 \\
\hline Error & Error message. & 1 \\
\hline
\end{tabular}

The project description file is not required when cave runs this command.

To add a \$Id \$ keyword to all of the files in the current directory (assuming there are no subdirectories), type:

cave header * 
To add the $\$$ Id $\$$ and $\$ \log \$$ keywords to all of the files in the current directory which have names starting with a or b, type:

$$
\text { cave header }-k \text { Id }-k \log [a-b]^{*}
$$

To add the \$Id\$ keyword to all of the files in the current directory, and have the comment line start with “\#”, type:

$$
\text { cave header }-c \text { \# * }
$$

NOTE: If you use the \$Log $\$$ keyword in a file, res places the log message in the file each time the file is committed. Unless told otherwise, it attempts to choose a comment character based on the file suffix. This may not be the character you want it to use. If this is the case, do the following:

- Commit the files.

- Go to the directory (or directories) in the repository that hold the files.

- Tell res what comment character(s) to use:

$$
\text { res -c"chars" file ... }
$$

There is no space between the $-c$ and the first quotation mark. 


\section{help - Get information on how to use cave}

This command to cave is used to get information on how to use the commands available in cave.

help [command]

command

Name of the cave command. The default is to provide a one line description of all the commands.

A description of the command, its options, and its functionality is returned. You might want to pipe the output through more:

cave help I more

Returns:

\begin{tabular}{|l|l|c|}
\cline { 2 - 3 } \multicolumn{1}{c|}{ Standard Out/Error } & exit value \\
\hline Success & Description of the command(s). & 0 \\
\hline Error & Error message. & 1 \\
\hline
\end{tabular}

The project description file is not required when cave runs this command. 


\section{history - Report on activities affecting a source repository}

This command to cave is a simple interface to the cvs history command. It is used to report on each use of cvs' checkout, commit, rtag, update, and release commands.

history [-d root_dir] [-report] [-flags] [-options] [-F list] [file...]

Synonym: hi

-d root_dir

Tail of a root directory name. If a project uses more than one root directory, the - $d$ command must be used. If the project uses only one root directory, the command is unnecessary.

One of the following -report arguments may be used to control what kind of report is generated. The default is -0 .

-xtype

$-\mathrm{c}$

$-\mathbf{m}$ module

$-0$

$-\mathrm{T}$

$-e$

Extract a particular set of record types. One or more types may be selected. They are:

o checkout

F release

T rtag

W update - working copy deleted because it was gone from the repository.

U update - working file copied from repository.

G update - merge was necessary, and it succeeded.

C update - merge was necessary, but collisions were detected.

M commit - file was modified.

A commit - file was first added.

R commit - file was removed.

For example, to see all files that were committed, use -XMAR.

Report each time commit was used (same as -xMAR).

Report on a particular module. More than one $-\mathbf{m}$ option may be used.

Report on modules which were checked out but not committed or released (default).

Report on all tags (same as -xT).

Everything (same as -xMACFROGWUT). 
One of the following -flags arguments may be used to constrain the report.

$-\mathbf{a}$

$-1$

$-\mathbf{w}$
Show data for all users. The default is to show data only for the user executing the history command.

Show last modification only.

Show only the records for modifications done from the same working directory where cave history is executing.

One or more of the following -options arguments may be used to constrain the report.

-b str

-D date

-p repository

-r rev

-t tag

-u name
Show data back to a record containing the string str in either the module name, the file name, or the repository path.

\section{Show data since date.}

Show data for a particular source repository. You can specify several $-p$ options on the same command line.

Show records referring to revisions since the revision or tag named rev appears in individual res files. Each file is searched for the revision or tag.

Show records since tag was last added to the history file. This differs from the $-r$ option above in that it reads only the history file, not the res files, and is much faster.

Show records for user name. The default is to show the records for one's self.

Some of the -report arguments accept a list of file names. The reports which accept files names are -c and -xWUGCMAR.

-F list

list is a file containing the names of files to be used with this command.

Each name must be in the same form as file.

Blank lines and leading blanks are allowed.

A "\#" signals the start of a comment.

Multiple lines are allowed. More than one entry may be on a line. If so, they must be blank delimited.

The -F option may be used in addition to or in place of file.

file

The name(s) may be the pathname relative to the root directory, or any substring contained therein.

It is necessary for the file history to exist in the CVSROOT directory in each root directory for this command to work properly. This file should be created at the time the root directory is set up. 
Returns:

\begin{tabular}{|l|l|c|}
\cline { 2 - 3 } \multicolumn{1}{c|}{} & Standard Out/Error & exit value \\
\hline Success & Report & 0 \\
\hline Error & Error message. & 1 \\
\hline
\end{tabular}

For example, if my user name is "me", any of the following will show which modules I have checked out without committing or releasing:

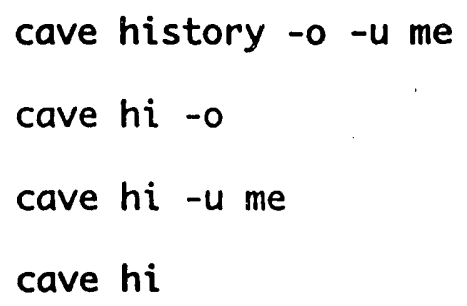

To show all the files the user named "other" has committed since the history file was created, type:

$$
\text { cave hi }-c-u \text { other }
$$

To show all the files you committed since Midnight, January 1, 1990:

$$
\text { cave hi -C -D 'Jan } 1 \text { 1990' }
$$

To show all the files committed since tag "TAG" was stored in the history file:

$$
\text { cave hi }-c-a-t \text { TAG }
$$

To show all the files committed since tag "TAG" was placed on the files:

$$
\text { cave hi }-c-a-r \text { TAG }
$$

History of user "user":

$$
\text { cave hi -e -u user }
$$

The project description file is required when cave runs this command. 


\section{lock - Create a lock}

This command to cave is used to create a lock file for a project.

lock [-m message] lock_type

-m message A quoted string containing a message to be included in the lock file when creating a lock. The default is no message.

lock_type

Type of lock. The types which normally are touched by the user are:

panic Emergency lock.

update General purpose lock.

The types which are created and removed within cave commands, and normally are not touched by the user are:

extract Lock while extracting information or files from the repository. This lock is created and removed from within any command that extracts things from the repository, such as checkout and diff. It is set so that the source repository doesn't change at the same time as files are being checked out or examined.

insert Lock while changing the repository. This lock is created and removed from within any command that changes the repository, such as commit and rtag.

If you want to lock the root directory while doing several operations, use the update lock instead of the insert lock. All cave commands that interact with a root directory check for the panic lock, the update lock, and the insert lock. Some also check for the extract lock.

\section{Returns:}

\begin{tabular}{|l|l|c|}
\cline { 2 - 3 } \multicolumn{1}{c|}{ Standard Out/Error } & exit value \\
\hline Success & $\begin{array}{l}\text { Completion message, or information on who owns the } \\
\text { lock, when it was set and any message it contains. }\end{array}$ & 0 \\
\hline Error & Error message. & 1 \\
\hline
\end{tabular}

The project description file is required when cave runs this command. 


\section{log - Display log information for files}

This command to cave is an interface to the rcs utility rlog. It is used to display log information about individual files in a local directory.

$\log [-1]$ [rlog-options] [-F list] [file ...]

Synonym: rlog

$-1$

rlog-options
Local. Do not recursively descend the directory structure. The default is to descend the directory struciure.

See the rlog(1) man page for full explanations of all the options. Some of the more useful options are:

-b Print information about the revisions on the default branch, normally the highest branch on the trunk.

-ddates Print information about revisions with a check in date/time in the ranges given by the semicolon-separated list of dates. A range of the forn $d 1<d 2$ or $d 2>d 1$ selects the revisions that were deposited between $d l$ and $d 2$ inclusive. A range of the form $<d$ or $d>$ selects all revisions dated $d$ or earlier. A range of the form $d<$ or $>d$ selects all revisions dated $d$ or later. A range of the form $d$ selects the single, latest revision dated $d$ or earlier. The date/time strings $d, d l$, and $d 2$ are in the free format explained in co(1). Quoting is normally necessary, especially for $<$ and $>$. Note that the separator is a semicolon.

-h Print only the rcs pathname, working pathname, head, default branch. access list, locks, symbolic names, and suffix.

-R Print only the name of the rcs file. This is convenient for translating a working pathname into a repository pathname.

$-r$ revisions] Prints information about revisions given in the comma- separated list of revisions and ranges. A range rev1:rev2 means revisions revl to rev2 on the same branch, :rev means revisions from the beginning of the branch up to and including rev, and rev: means revisions starting with rev to the end of the branch containing rev. An argument that is a branch means all revisions on that branch. A range of branches means all revisions on the branches in that range. A branch followed by a means the 
-F list

file latest revision in that branch. A bare $-r$ with no revisions means the latest revision on the default branch, normally the trunk.

-w[logins] Prints information about revisions checked in by users with login names appearing in the comma-separated list logins. If logins is omitted, the user's login is assumed.

list is a file containing the names of files to be used with this command.

Each name must be in the same form as file. Blank lines and leading blanks are allowed. A "\#" signals the start of a comment. Multiple lines are allowed. More than one entry may be on a line. If so, they must be blank delimited.

The -F option may be used in addition to or in place of file.

The name of a file or directory which was placed in the current local directory by cvs. The default is to report on all files and directories.

The files to check must exist in the current local directory or a subdirectory. The current directory must have been created by cave checkout or cvs checkout (it contains a CVS directory).

Without options, rlog prints the following information for each res file: rcs pathname, working pathname, head (i.e., the number of the latest revision on the trunk), default branch, access list, locks, symbolic names, suffix, total number of revisions, number of revisions selected for printing, and descriptive text. This is followed by entries for the selected revisions in reverse chronological order for each branch. For each revision, rlog prints revision number, author, date/time, state, number of lines added/deleted (with respect to the previous revision), locker of the revision (if any), and log message. All times are displayed in Coordinated Universal Time (UTC). The options above restrict this output.

\section{Returns:}

\begin{tabular}{|l|l|c|}
\cline { 2 - 3 } \multicolumn{1}{c|}{} & Standard Out/Error & exit value \\
\hline Success & Report. & 0 \\
\hline Error & Error message. & 1 \\
\hline
\end{tabular}

The project description file is required when cave runs this command. 


\section{pack - Collect files together for distribution}

This command to cave is used to collect files together for distribution to another machine.

pack [co_opts] [pack_opts]

:The pack_opts are:

$-\mathrm{C}$

-d dir

$-\mathbf{w}$ from

$-\mathbf{T}$

$-\mathbf{Z}$

-i distrib

-F list
A flag to calculate checksums for all of the files in dir (except for the tag file). The results are placed in a file named dir/sum_file.

The name of a directory to hold the collected files. The default is to name it "Pack".

If the name starts with a slash, it is assumed to be an absolute pathname. If it does not start with a slash, it is assumed to be relative to the project directory if one can be found, otherwise it is relative to the current directory. The path up to the tail of the directory name must already exist. The tail does not have to exist, but it may. If it does exist, the directory must be empty.

If the $-\mathbf{T}$ option is used, the contents of the directory are replaced with a tar file. If the $-\mathrm{Z}$ option is used, the contents of the directory are replaced with a compressed tar file.

Location from which to collect files. The choices are:

both Get files from the local workspace, and get any missing ones from the source repository.

local Get files from the local workspace only.

repo Get files from the source repository.

The default is to use both.

Place the collected files in a tar file.

Place the collected files in a compressed tar file.

The name of a distribution, as defined with the pack keyword in the project description file. This argument may be combined with the path argument. The default, if neither is used, is to include all the files which are under cave control and are a part of the project.

list is a file containing the names of paths to be used with this command.

Each name must be in the same form as path.

Blank lines and leading blanks are allowed. 
path

A "\#" signals the start of a comment.

Multiple lines are allowed. More than one entry may be on a line. If so, they must be blank delimited.

The -F option may be used in addition to or in place of path.

$-\mathrm{x}$ other

The name of a directory or file which is within one of the root directories, yet not under cave control. In the simplest case, when a project only uses one root directory, other must be the pathname of a file or subdirectory relative to the root directory.

If a project uses more than one root directory, other must start with the tail of the root directory pathname, and must be followed by the relative pathname of a file or subdirectory.

If other ends with a directory name, all files contained within that directory are included.

A cave controlled file may be requested here. If so, the actual ", $v$ " file from the repository is copied.

Note: These files are always collected from the root directory. The "-w from" argument has no effect.

A pathname. In the simplest case, when a project only uses one root directory, it must be one of the following:

- A key, as defined in the modules file for this root directory.

\section{module}

- A pathname which must start with the tail of a source repository name. It may continue, to include the name of a subdirectory or file.

$$
\text { repository[/dir[/...]] }
$$

If a project uses more than one root directory, the pathname must start with the tail of the absolute root directory pathname. It may continue with:

- A key, as defined in the modules file for this root directory.

$$
\text { root_dir[/module] }
$$

- The relative pathname of a source repository and its contents.

$$
\text { root_dir[/repository[/dir[/...]]] }
$$

If path ends with a source repository or subdirectory name, all files contained within that source repository or directory are included (unless the -1 option was used).

The files must exist in the source repository, and be under 
cave control. Do not include the ",v" suffix when specifying file names. This argument may be combined with the -i argument. The default, if neither is used, is to include all the files which are under cave control and are a part of the project.

The co_opts are passed to the cvs checkout command if any files need to be retrieved from the source repository.

The most common co_opts are:

-D date

$-\mathbf{f}$

$-1$

$-\mathbf{P}$

$-\mathbf{p}$

$-\mathbf{Q}$

$-\mathbf{q}$

$-\mathbf{r}$ tag
Use the revision that was checked in at or before the specified date argument. The date is a single argument, date description specifying a date in the past. The formats are similar to those described in co(1). Examples of valid date specifications include:

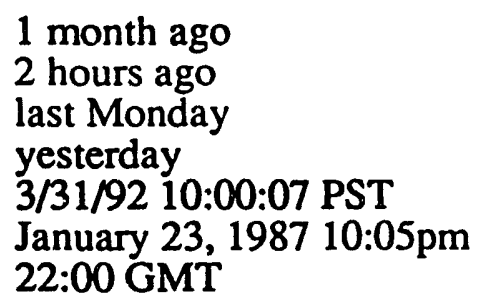

Forces the specified tag to match. Normally, if a tag is specified and the requested file does not contain the tag, the file is ignored. The -f option causes any file which does not contain the tag to be retrieved even when there is no match for the tag or date.

Local. Do not recursively descend the directory structure.

Prune directories that are empty. This does not remove the directory from the repository, only from your checked out copy.

Pipe the files to standard output, rather than writing them to the local directory.

Causes the command to be really quiet.

Causes the command to be somewhat quiet.

Use the revision of the file which was marked with the symbolic tag "tag". To avoid getting empty directories, combine this option with the -P option:

$$
\text { -P -r tag }
$$

The default is to check out the latest version. 
The more advanced co_opts are:

$-\mathbf{A}$

-j branch

-k kflag

$-\mathbf{n}$

$-S$

Reset any sticky tags, dates or -k options. (If you get a working file using one of the $-\mathbf{r},-\mathbf{D}$, or -k options, cvs remembers the corresponding tag, date or kflag and continues using it on future updates. Use the -A option to make cvs forget these specifications.)

Merges the changes between two different branches of development. For more information, read the cvs man pages.

Alter the default processing of res keywords; all the -k options described in res(1) are available. Your kflag specification is "sticky" when you use it to create a private copy of a source file. That is, when you use this option with the checkout or update commands, cvs associates your selected kflag with the file, and continues to use it with future update commands on the same file until you specify otherwise. The kflag option is stored in the source file and can be changed with cvs admin.

Causes the command not to run any checkout program as specified in the modules file.

Display per-module status information within the modules file.

Returns:

\begin{tabular}{|l|l|c|}
\cline { 2 - 3 } \multicolumn{1}{c|}{} & Standard Out/Error & exit value \\
\hline Success & Message. & 0 \\
\hline Error & Error message. & 1 \\
\hline
\end{tabular}

The project description file is required when cave runs this command.

What to pack is determined by first collecting all the lists from -i distrib, - F list, $-\mathbf{x}$ other, and path. These are sorted into a list of cave controlled files and a list of other files. Any duplications within a given list are removed.

Files are moved into the Pack directory in the following order:

- If from is both or repo, the files are checked out from the repository.

- If from is both or local, the local files are moved over using tar. There is no checking for conflicts or updates. Any CVS directories present in the local workspace are automatically removed from the distribution.

Note: All files contained in any requested local directories are included, whether or not they are under cave control.

- If $\mathbf{a}-\mathbf{x}$ option is used in the execute line or in a requested distribution, those files are moved over using tar.

- If the -c option is used, a checksum file is created. 
- If there is a tag file defined by a tag line in the project description file, the file is included with the collected files.

Note: If the - $r$ option was used to check out a particular version, the tag in the tag file may not match the tag that was given for $-r$.

Each root directory is assumed to contain the cvs administrative directory called CVSROOT. This directory and its files may be packed, but it must be explicitly requested in path:

\begin{tabular}{|l|l|l|}
\cline { 2 - 3 } \multicolumn{1}{c|}{} & One root directory & More than one root directory \\
\hline Path to use with pack & CVSROOT & root_dir/CVSROOT \\
modules & root dir/modules \\
\hline $\begin{array}{l}\text { File/directory created in } \\
\text { local project directory }\end{array}$ & $\begin{array}{l}\text { CVSROOT } \\
\text { CVSROOT/modules }\end{array}$ & $\begin{array}{l}\text { CVSROOT.root_dir } \\
\text { CVSROOT.root dir/modules }\end{array}$ \\
\hline
\end{tabular}




\section{print - Print text files}

This command to cave is used to send text files to a printer.

print [co_opts] [-w from] [-I] [-c] [-p “lpr_line"] [...] [-F list] [path ...]

Synonym: Ipr

$-w$ from

Location from which to collect files. The choices are:

both Get files from the local workspace, and get any missing ones from the source repository.

local Get files from the local workspace only.

repo Get files from the source repository.

The default is to use both.

$-1$

$-c$

-p "lpr_line"

-F list

path
Local. Do not recursively descend the directory structure.

"cat" the files contained in the same directory before printing them.

A quoted string containing a command line to send to the lpr print spooler. The default is to send the listings as given in the print lines in the project description file.

list is a file containing the names of paths to be used with this command.

Each name must be in the same form as path.

Blank lines and leading blanks are allowed.

A "\#” signals the start of a comment.

Multiple lines are allowed. More than one entry may be on a line. If so, they must be blank delimited.

The -F option may be used in addition to or in place of path.

A pathname. In the simplest case, when a project only uses one root directory, it must be one of the following:

A key, as defined in the modules file for this root directory.

module

A pathname which must start with the tail of a source repository name. It may continue, to include the name of a subdirectory or file. 


$$
\text { repository[/dir[/...]] }
$$

If a project uses more than one root directory, the pathname must start with the tail of the absolute root directory pathname. It may continue with:

- A key, as defined in the modules file for this root directory.

$$
\text { root_dir[/module }]
$$

- The relative pathname of a source repository and its contents.

$$
\text { root_dir[/repository[/dir[/...]]] }
$$

If path ends with a source repository or subdirectory name, all files contained within that source repository or directory are included (unless the -l option was used).

The files must exist in the source repository, and be under cave control. Do not include the " $v$ " suffix when specifying file names. The default is to print all the files which are under cave control and are a part of the project.

The co_opts are passed to the cvs checkout command if any files need to be retrieved from the source repository.

The most common co_opts are:

-D date

-f

$-\mathbf{P}$
Use the revision that was checked in at or before the specified date argument. The date is a single argument, date description specifying a date in the past. The formats are similar to those described in co(1). Examples of valid date specifications include:

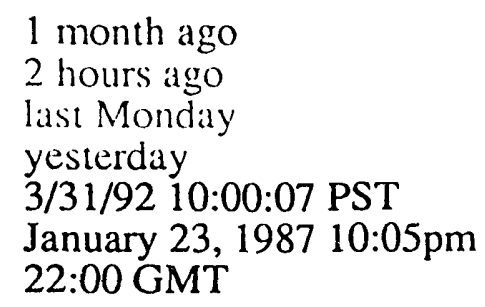

Forces the specified tag to match. Normally, if a tag is specified and the requested file does not contain the tag, the file is ignored. The -f option causes any file which does not contain the tag to be retrieved even when there is no match for the tag or date.

Prune directories that are empty. This does not remove the directory from the repository, only from your checked out copy. 

-p Pipe the files to standard output, rather than writing them to the local directory.
-Q Causes the command to be really quiet.
-q Causes the command to be somewhat quiet.
-r tag Use the revision of the file which was marked with the symbolic tag "tag". To avoid getting empty directories, combine this option with the -P option:

$$
\text { -P -r tag }
$$

The default is to check out the latest version.

The more advanced co_opts are:

$-\mathbf{A}$

-j branch

-k kflag

$-n$

$-\mathbf{S}$
Reset any sticky tags, dates or $-k$ options. (If you get a working file using one of the $-r,-D$, or $-k$ options, cvs remembers the corresponding tag, date or kflag and continues using it on future updates. Use the -A option to make cvs forget these specifications.)

Merges the changes between two different branches of development. For more information, read the cvs man pages.

Alter the default processing of res keywords; all the -k options described in res(1) are available. Your kflag specification is "sticky" when you use it to create a private copy of a source file. That is, when you use this option with the checkout or update commands, crs associates your selected kflag with the file, and continues to use it with future update commands on the same file until you specify otherwise. The kflag option is stored in the source file and can be changed with cvs admin.

Causes the command not to run any checkout program as specified in the modules file.

Display per-module status information within the modules file.

Returns:

\begin{tabular}{|l|l|c|}
\cline { 2 - 3 } \multicolumn{1}{c|}{} & Standard Out/Error & exit value \\
\hline Success & Message & 0 \\
\hline Error & Error message. & 1 \\
\hline
\end{tabular}

The project description file is required when cave runs this command.

Files are collected for printing in the following order: 
- If from is both or repo, the files are checked out from the repository.

- If from is both, the local files are moved over using tar. There is no checking for conflicts or updates. If from is local, the local files are used directly. Any CVS directories present in the local workspace are automatically ignored. All files contained in any requested local directories are included, whether or not they are under cave control.

Each root directory is assumed to contain the cvs administrative directory called CVSROOT. This directory and its files may be printed, but it must be explicitly requested in path.:

\begin{tabular}{|l|l|l|}
\cline { 2 - 3 } \multicolumn{1}{c|}{} & One root directory & More than one root directory \\
\hline Path to use with print & CVSROOT & root_dir/CVSROOT \\
modules & root dir/modules \\
\hline $\begin{array}{l}\text { File/directory created in } \\
\text { local project directory }\end{array}$ & $\begin{array}{l}\text { CVSROOT } \\
\text { CVSROOT/modules }\end{array}$ & $\begin{array}{l}\text { CVSROOT.root_dir } \\
\text { CVSROOT.root dir/modules }\end{array}$ \\
\hline
\end{tabular}




\section{release - Cancel a check out}

This command to cave is used to safely cancel the effects of a check out.

release [-dQq] [-F list module [...]

Synonym: rel

$-d$

$-\mathbf{Q}$

$-\mathbf{q}$

-F list

Delete the files, if the command succeeds.

Causes the command to be really quiet.

Causes the command to be somewhat quiet.

list is a file containing the names of modules to be used with this command.

Each name must be in the same form as module.

Blank lines and leading blanks are allowed.

A “\#” signals the start of a comment.

Multiple lines are allowed. More than one entry may be on a line. If so, they must be blank delimited.

The $-F$ option may be used in addition to or in place of module.

module

A key, as defined in the modules file.

This command isn't strictly necessary, as a working directory can simply be deleted. It does provide some useful features, however. This command checks that no uncommitted changes are present, that you are executing it from inside or immediately above a cvs working directory, and that the repository name is good. It also leaves a record of its execution in the history file, which simply deleting the files will not do.

This command must be called from within or below the project directory. If it is called from within the project directory, it will descend at least one level.

Returns:

\begin{tabular}{|l|l|c|}
\cline { 2 - 3 } \multicolumn{1}{c|}{ Standard Out/Error } & exit value \\
\hline Success & Message & 0 \\
\hline Error & Error message. & 1 \\
\hline
\end{tabular}

The project description file is required when cave runs this command. 


\section{remove - Remove a file}

This command to cave is used to remove files from a source repository.

remove [-R] [-F list] [file ...]

Synonym: rm, delete

$-\mathbf{R}$

-F list
A flag to recursively descend into any directory given in the-file list, and remove its files. The default is to not descend. The directory itself cannot be removed.

list is a file containing the names of files to be used with this command.

Each name must be in the same form as file.

Blank lines and leading blanks are allowed.

A "\#" signals the start of a comment.

Multiple lines are allowed. More than one entry may be on a line. If so, they must be blank delimited.

The -F option may be used in addition to or in place of file.

file

The name of a file or directory which was placed in the current working directory by cave. The default is to remove all files in the current directory which are under cave control. Specifying a directory without using the - $R$ option causes nothing to happen to the contents of the directory. The directory itself cannot be removed. Any directory involved in the remove must have been created by cave checkout or cvs checkout (it contains a CVS directory).

Marks the specified files as removed on purpose from the current directory in the source repository directory. The files may have been deleted already from the local directory. If they were not deleted, this command deletes them. In either case, they are marked to be removed. The files are not actually removed from the source repository until a commit is done. At that time, they are moved into the Attic directory.

Use add to undo a remove command, unless the change has already been committed.

Returns:

\begin{tabular}{|l|l|c|}
\cline { 2 - 3 } \multicolumn{1}{c|}{ Standard Out/Error } & exit value \\
\hline Success & Message & 0 \\
\hline Error & Error message. & 1 \\
\hline
\end{tabular}

The project description file is required when cave runs this command. 


\section{reserve - Reserve a file or directory}

This command to cave is used to reserve a file or directory so that no other user may modify it.

reserve $[-\mathrm{m}$ message $][-\mathrm{F}$ list $]$ path $[\ldots]$

\section{Synonym: res}

-m message

-F list

path
A quoted string containing a message to be included when reserving. The default is no message.

list is a file containing the names of paths to be used with this command.

Each name must be in the same form as path.

Blank lines and leading blanks are allowed.

A "\#" signals the start of a comment.

Multiple lines are allowed. More than one entry may be on a line. If so, they must be blank delimited.

The -F option may be used in addition to or in place of path.

A pathname.

In the simplest case, when a project only uses one root directory, it must start with the tail of a source repository name. It may continue, to include the name of a subdirectory or file.

$$
\text { repository[/dir }[/ \ldots]]
$$

If a project uses more than one root directory, the pathname must start with the tail of the absolute root directory pathname. It may continue with the relative pathname of a source repository and its contents.

$$
\text { root_dir[/repository[/dirl/...]]] }
$$

The files must exist in the source repository, and be under cave control. Do not include the ", $v$ " suffix when specifying file names.

You may be thinking that this command runs against the philosophy and purpose of cvs. I agree completely.

Note that the reserve command is entirely independent of res locking.

Run cave what reserve to see what is currently reserved. 
Let us consider a directory to be of higher rank than one of its subdirectories or files.

When you attempt to reserve a directory and/or file, the rules of precedence are as

follows.

- Error if it is not part of the project.

- Error if it is already reserved by someone else.

- Error if one of its parent directories is already reserved by someone else.

- Error if one of its subdirectories or files is already reserved by someone else.

- If more than one path is given and one outranks another, only the higher ranking path is reserved.

- If path is the same as one already reserved by you, keep the old reservation.

- If path is outranked by something already reserved by you, keep the old reservation.

- If path outranks something already reserved by you, remove the old reservation. Reserve path.

- If there are no conflicts, reserve path.

Returns:

\begin{tabular}{|l|l|c|}
\cline { 2 - 3 } \multicolumn{1}{c|}{ Standard Out/Error } & exit value \\
\hline Success & Message & 0 \\
\hline Error & Error message. & 1 \\
\hline
\end{tabular}

The project description file is required when cave runs this command. 


\section{rtag - Assign symbolic tags to sources}

This command to cave is used to assign symbolic tags to particular, explicitly specified source versions in the repository.

rtag [-afinQqR] [-b] [-d] [-r tag I-D date] [-F list] symbolic_tag module ...

Synonym: rfreeze

$\boldsymbol{- a}$

$-\mathbf{f}$

$-1$

$-\mathbf{n}$

$-\mathbf{Q}$

$-9$

$-\mathbf{R}$

-b

$-d$

$-\mathrm{D}$ date
Look in the Attic for removed files that contain the specified tag. The tag is removed from these files, which makes it possible to reuse a symbolic tag.

Forces the specified tag to match. Normally, if a tag is specified and the requested file does not contain the tag, the file is ignored. The -f option causes any file which does not contain the tag to be used even when there is no match for the tag or date.

Local. Do not recursively descend the directory structure. The default is to descend the direstory structure.

Causes the command not to run any tag program as specified in the modules file.

Causes the command to be really quiet.

Causes the command to be somewhat quiet.

Recursively descend into any subdirectories. This is the default.

Make the tag a "branch" tag, allowing concurrent, isolated development.

Remove a symbolic tag from files.

Use the revision that was checked in at or before the specified date argument. The date is a single argument, date description specifying a date in the past. The formats are similar to those described in co(1). Examples of valid date specifications include:

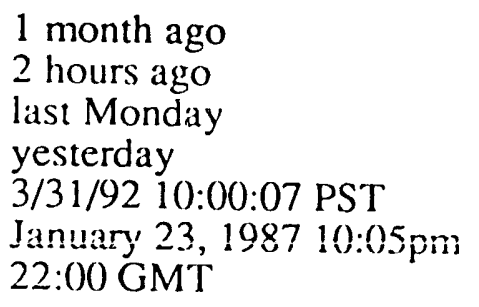


-r tag

- F list

symbolic_tag

module
Use this to tag only those files that already contain a certain symbolic tag. This is useful in combination with the -d option to rename a tag.

list is a file containing the names of modules to be used with this command.

Each name must be in the same form as module.

Blank lines and leading blanks are allowed.

A "\#" signals the start of a comment.

Multiple lines are allowed. More than one entry may be on a line. If so, they must be blank delimited.

The -F option may be used in addition to or in place of module.

The tag must start with a letter, and must not contain a semicolon, colon, dollar sign, at sign, comma or period (;:\$@,.).

A key, as defined in the modules file for this root directory. If a project uses more than one root directory, module must start with the tail of the absolute root directory pathname and continue with the module, as defined in the modules file:

\section{root_dir/module}

This command may be called from any local directory. It is similar to tag, but no local files need to be present.

Returns:

\begin{tabular}{|l|l|c|}
\cline { 2 - 3 } \multicolumn{1}{c|}{} & Standard Out/Error & exit value \\
\hline Success & Message & 0 \\
\hline Error & Error message. & 1 \\
\hline
\end{tabular}

The project description file is required when cave runs this command. 


\section{status - Report on the current status of files}

This command to cave is used to report what has been done to files in the working directory and the source repository.

status [-IR] [-v] [-F list] [file ...]

Synonym: stat

$-1$

$-\mathbf{R}$

$-\mathbf{v}$

-F list
Local. Do not recursively descend the directory structure. The default is to descend the directory structure.

Recursively descend into any subdirectories, unless file names are given. This is the default.

Display symbolic tags in the report.

list is a file containing the names of files to be used with this command.

Each name must be in the same form as file.

Blank lines and leading blanks are allowed.

A "\#" signals the start of a comment.

Multiple lines are allowed. More than one entry may be on a line. If so, they must be blank delimited.

The -F option may be used in addition to or in place of file.

file

The name of a file or directory which was placed in the current local directory by cvs. The default is to report on all files and directories.

This command must be called from within or below the project directory. If it is called from within the project directory, it will descend at least one level.

Returns:

\begin{tabular}{|l|l|c|}
\cline { 2 - 3 } \multicolumn{1}{c|}{} & Standard Out/Error & exit value \\
\hline Success & Report. & 0 \\
\hline Error & Error message. & 1 \\
\hline
\end{tabular}

The project description file is required when cave runs this command. 


\section{tag - Assign symbolic tags to sources}

This command to cave is used to assign symbolic tags to source versions in the repository. The files to be tagged are determined by your local files.

$\operatorname{tag}[-1 Q q R][-b][-d]$ [-F list] symbolic_tag [file...]

\section{Synonym: freeze}

$-1$

$-\mathbf{Q}$

$-\mathbf{q}$

$-\mathbf{R}$

$-\mathbf{b}$

$-\mathbf{d}$

-F list

symbolic:_ag

file
Local. Do not recursively descend the directory structure. The default is to descend the directory structure.

Causes the command to be really quiet.

Causes the command to be somewhat quiet.

Recursively descend into any subdirectories. This is the default.

Make the tag a "branch" tag, allowing concurrent, isolated development.

Remove a symbolic tag from files.

list is a file containing the names of files to be used with this command.

Each name must be in the same form as file.

Blank lines and leading blanks are allowed.

A "\#" signals the start of a comment.

Multiple lines are allowed. More than one entry may be on a line. If so, they must be blank delimited.

The -F option may be used in addition to or in place of file.

The tag must start with a letter, and must not contain a semicolon, colon, dollar sign, at sign, comma or period (;:\$@,.).

Name of a local directory or file under cave control. The default is to include all the files and directories in the current directory.

This is similar to rtag, but the files to be tagged in the repository are determined by the files in your local directory.

This command must be called from within or below the project directory. If it is called from within the project directory, it will descend at least one level.

Returns: 


\begin{tabular}{|l|l|c|}
\cline { 2 - 3 } \multicolumn{1}{c|}{} & Standard Out/Error & exit value \\
\hline Success & Message & 0 \\
\hline Error & Error message. & 1 \\
\hline
\end{tabular}

The project description file is required when cave runs this command. 


\section{unlock - Remove a lock}

This command to cave is used to remove a lock file for a project.

unlock lock_type I-n lock_name

lock_type Type of lock. The types which normally are touched by the user are:

panic Emergency lock.

update General purpose lock.

The types which are created and removed within cave commands, and normally are not touched by the user are:

extract Lock while extracting information or files from the repository. This lock is created and removed from within any command that extracts things from the repository, such as checkout and diff. It is set so that the source repository doesn't change at the same time as files are being checked out or examined.

insert Lock while changing the repository. This lock is created and removed from within any command that changes the repository, such as commit and rtag.

-n lock_name

The actual name of a lock file. The naming convention is described in the chapter "Project Description File". To determine the names of all lock files, run cave what lock.

Only the owner can remove a lock file. All cave commands that interact with a root directory check for the panic lock, the update lock, and the insert lock. Some also check for the extract lock.

Returns:

\begin{tabular}{|l|l|c|}
\cline { 2 - 3 } \multicolumn{1}{c|}{ Standard Out/Error } & exit value \\
\hline Error & $\begin{array}{l}\text { Completion message, or information on who owns the } \\
\text { lock, when it was set and any message it contains. }\end{array}$ & 0 \\
\hline & Error message. & 1 \\
\hline
\end{tabular}

The project description file is required when cave runs this command. 


\section{unreserve - Unreserve a file or directory}

This command to cave is used to unreserve a file or directory.

unreserve $[-\mathrm{F}$ list $]$ path $[\ldots]$

Synonym: unres

-F list

path list is a file containing the names of paths to be used with this command.

Each name must be in the same form as path.

Blank lines and leading blanks are allowed.

A "\#" signals the start of a comment.

Multiple lines are allowed. More than one entry may be on

a line. If so, they must be blank delimited.

The -F option may be used in addition to or in place of path.

A pathname.

In the simplest case, when a project only uses one root directory, it must start with the tail of a source repository name. It may continue, to include the name of a subdirectory or file.

$$
\text { repository[/dir[/...]] }
$$

If a project uses more than one root directory, the pathname must start with the tail of the absolute root directory pathname. It may continue with the relative pathname of a source repository and its contents.

$$
\text { root_dir[/repository[/dir[/...]]] }
$$

The files must exist in the source repository, and be under cave control. Do not include the ",v" suffix when specifying file names.

You think that this command runs against the philosophy and purpose of cvs. I agree completely. Enough said.

Note that the reserve command is entirely independent of rcs locking.

Run cave what reserve to see what is currently reserved.

Let us consider a directory to be of higher rank than one of its subdirectories or files. When you attempt to unreserve a directory and/or file, the rules of precedence are as follows. 
- Error if it is not part of the project.

- Error if it was reserved by someone else.

- Error if one of its parent directories or subdirectories or files was reserved by someone else.

- If more than one path is given and one outranks another, only the higher ranking path is used.

- If path is the same as one already reserved by you, unreserve it.

- If path is outranked by something reserved by you, ignore path.

- If path outranks anything reserved by you, remove all the outranked reservations.

Returns:

\begin{tabular}{|l|l|c|}
\cline { 2 - 3 } \multicolumn{1}{c|}{} & Standard Out/Error & exit value \\
\hline Success & Message & 0 \\
\hline Error & Error message. & 1 \\
\hline
\end{tabular}

The project description file is required when cave runs this command. 


\section{update - Make files current}

This command to cave is used to make any local files current with any that have been modified in the source repository since the local files were checked out.

update [-AdflPpQq] [-j branch] [-r tag I-D date] [-I name [-I name...]] [-F list] [file ...]

$-\mathbf{A}$

$-d$

$-\mathbf{f}$

-j branch

$-1$

$-\mathbf{P}$

$-p$

$-Q$

$-\mathbf{q}$

-D date
Reset any sticky tags, dates or -k options. (If you get a working file using one of the $-r,-D$, or $-k$ options, cvs remembers the corresponding tag, date or kflag and continues using it on future updates. Use the -A option to make cvs forget these specifications.)

Create any directories that exist in the repository if they are missing from the working directory.

Forces the specified tag to match. Normally, if a tag is specified and the requested file does not contain the tag, the file is ignored. The -f option causes any file which does not contain the tag to be used even when there is no match for the tag or date.

Merges the changes between two different branches of development. For more information, read the cvs man pages.

Only process the current directory, ignoring any subdirectories.

Prune directories that are empty. This does not remove the directory from the repository, only from your checked out copy.

Pipe the files to standard ouput, rather than writing them to the locial directory.

Causes the command to be really quiet.

Causes the command to be somewhat quiet.

Use the revision that was checked in at or before the specified date argument. The date is a single argument, date description specifying a date in the past. The formats are similar to those described in co(1). Examples of valid date specifications include:

1 month ago

2 hours ago

last Monday

yesterday 


\begin{tabular}{|c|c|c|}
\hline & \multicolumn{2}{|c|}{$\begin{array}{l}\text { 3/31/92 10:00:07 PST } \\
\text { January 23, 1987 10:05pm } \\
\text { 22:00 GMT }\end{array}$} \\
\hline$-\mathbf{r}$ tag & \multicolumn{2}{|c|}{$\begin{array}{l}\text { Use the revision number or symbolic tag specified by the } \\
\text { tag argument instead of the usual "head" revision. }\end{array}$} \\
\hline -I name & \multicolumn{2}{|c|}{$\begin{array}{l}\text { Ignore files whose names match name. By default, update } \\
\text { ignores files whose names match any of the following: }\end{array}$} \\
\hline & $\begin{array}{l}\text { RCSLOG } \\
\text { CVS* } \\
\text { tags } \\
\text {.make.state } \\
\text { * \#* } \\
\text { *.old *.bak } \\
\text { *.a *.o }\end{array}$ & 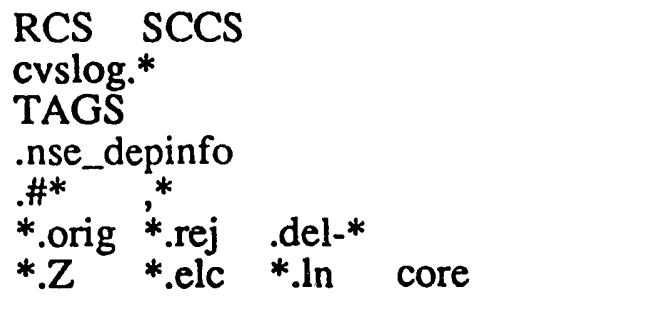 \\
\hline & \multicolumn{2}{|c|}{ Use "-I !" to avoid ignoring any files at all. } \\
\hline -F list & \multicolumn{2}{|c|}{$\begin{array}{l}\text { list is a file containing the names of files to be used with } \\
\text { this command. } \\
\text { Each name must be in the same form as file. } \\
\text { Blank lines and leading blanks are allowed. } \\
\text { A "\#" signals the start of a comment. } \\
\text { Multiple lines are allowed. More than one entry may be on } \\
\text { a line. If so, they must be blank delimited. } \\
\text { The -F option may be used in addition to or in place of file. }\end{array}$} \\
\hline file & \multicolumn{2}{|c|}{$\begin{array}{l}\text { The name of a file or directory which was placed in the } \\
\text { current local directory by cvs or cave. If no file name is } \\
\text { given, all the files and directories in the current directory } \\
\text { are updated. }\end{array}$} \\
\hline
\end{tabular}

This command must be called from within or below the project directory. If it is called from within the project directory, it will descend at least one level.

The current directory and any subdirectories are brought up-to-date, unless a list of files is given or the -1 option used.

Any files which are in your local directory and were not changed by you are updated with any changes in the source repository. If a file was changed by you, and was also modified in the source repository, your file is copied to a file name that starts with ".\#". The source repository file and your file are then merged together. The changed lines are bracketed by lines containing " $>>>$ " and " $<<<$ ". The revision number at the top of the file, as contained in the "\$Id\$" string is modified to match the one in the source repository.

Note: It is up to you to resolve any conflicts. As far as cv's is concerned, your local file is now up-to-date.

Output is generated by cvs when updating the files to describe what actions are being taken: 
U file

A file

R file

M file

C file

? file
The specified file was copied directly out of the source repository. This was done because one of the following was true:

- You had checked out an entire directory, and the file exists in the repository copy of the directory, but the file did not exist in the local workspace.

- The file did exist, but you did not change it, and a newer revision had been placed in the root directory.

The file has been added locally by you, via the add command, but still needs to be committed to the source repository.

The file has been removed locally by you, via the remove command, but the change still needs to be committed to the source repository.

The file has been modified locally by you.

The file has been modified locally by you, and in the mean time was modified in the source repository. The merge process that is described above is performed.

The file is in your working directory, but does not correspond to anything in the source repository, and is not in the list of files for the update to ignore.

Returns:

\begin{tabular}{|l|l|c|}
\cline { 2 - 3 } \multicolumn{1}{c|}{ Standard Out/Error } & exit value \\
\hline Success & Message & 0 \\
\hline Error & Error message. & 1 \\
\hline
\end{tabular}

The project description file is required when cave runs this command.

Each root directory is assumed to contain the cvs administrative directory called CVSROOT. If this directory and its files were checked out with cave, they were placed in a directory named CVSROOT (if there is only one root directory in the project) or CVSROOT.root dir (if there is more than one root directory in the project). This directory may be updated, but it must be explicitly requested in file. 


\section{verify - Check the project description file}

This command to cave is used to check a project description file for correctness.

verify

All the error checking that is done whenever cave reads in a project description file is performed. It tries to find as many errors as possible before exiting.

Returns:

\begin{tabular}{|l|l|c|}
\cline { 2 - 3 } \multicolumn{1}{c|}{} & Standard Out/Error & exit value \\
\hline Success & Message stating the file was verified. & 0 \\
\hline Error & Error message(s). & 1 \\
\hline
\end{tabular}

The project description file is required when cave runs this command. 


\section{version - Report cave's version number}

This command to cave is used to report what version number of cave is in use. version

Synonym: vers

Returns:

\begin{tabular}{|l|l|c|}
\cline { 2 - 3 } \multicolumn{1}{c|}{} & Standard Out/Error & exit value \\
\hline Success & Version number & 0 \\
\hline Error & Error message(s). & 1 \\
\hline
\end{tabular}

The project description file not is required when cave runs this command. 


\section{what - Get information about a project}

This command to cave is used to get information about a project from its project description file, and related files.

what [is I are] key [args]

The optional words "is" and "are" in the command are simply a way to make the command look more like a sentence.

One key is t.sed to descrite the type of information desired. Only the first three characters of each key are checked.

The possible key words are:

access

dirs $[\operatorname{dir} \ldots]$

files $[$ file ...]
A list of users with access to this project. See access in the chapter "Project Description File" for more information.

A list of directories which are under cave control in the project. The Attic directories are not included. If dir is not given, all the directories are listed.

When a project uses only one root directory, dir is a pathname relative to the root directory:

$$
\text { repository[/dir[/...]] }
$$

When a project uses more than one root directory, dir must start with the tail of the absolute root directory pathname:

$$
\text { rout_dir[/repository[/dir[/...]]] }
$$

Regular expressions may be used in dir, if they are escaped with a backslash.

The list of returned directories is in the same format as dir.

A list of files which are under cave control in the project. Files in the Attic directories are not included. If file is not given, all the files are listed. The ",v" suffix is removed. When a project uses only one root directory, file is a pathname relative to the root directory:

$$
\text { repository[/dir[/...]] }
$$

When a project uses more than one root directory, file must start with the tail of the absolute root directory pathname:

$$
\text { root_dirl/repositoryl/dirl/...]]] }
$$

Regular expressions may be used in file, if they are escaped 
with a backslash.

The list of returned files is in the same format as file.

group

The name of the group which owns the project. See group in the chapter "Project Description File" for more information.

lock

The types and current settings of any lock files defined for this project. See lock in the chapter "Project Description File" for more information.

mail

A list of users to whom mail is sent when files are committed to this project. See mail in the chapter "Project Description File" for more information.

pack

The distribution names and the directories and files which comprise each distribution. See pack in the chapter "Project Description File" for more information.

print

Command lines that are used with the Ipr print spooler. See print in the chapter "Project Description File" for more information.

project

The name of this project. See project in the chapter "Project Description File" for more information.

repository

Absolute pathnames of source repositories used by this project. See repository in the chapter "Project Description File" for more information.

reserve

The names of any directories and files currently reserved for this project. See reserve in the chapter "Project Description File" for more information.

root_dir

Absolute pathnames of root directories used by this project. See root dir in the chapter "Project Description File" for more information.

tag

Returns the current value of the tag as it exists in the tag file. See tag in the chapter "Project Description File" for more information.

Since only the first three characters of key are checked, all of the following are equivalent:
cave what is reserved
cave what res
cave what reserve
cave what are restoots

Returns: 


\begin{tabular}{|l|l|c|}
\cline { 2 - 3 } \multicolumn{1}{c|}{} & Standard Out/Error & exit value \\
\hline Success & Value depending on the key word used. & 0 \\
\hline Error & Error message. & 1 \\
\hline
\end{tabular}

The project description file is required when cave runs this command. 


\section{Other CAVEMAN Tools}

There are other tools which are part of the CAVEMAN system. They are described on the following pages. 


\section{cmget}

NAME

cmget - a simple interface to ftp.

\section{SYNOPSIS}

cmget $-\mathrm{m}$ machine $[-\mathrm{d}$ directory] $[-\mathrm{v}][-\mathrm{b}]$ file ...

\section{DESCRIPTION}

cmget provides a simple interface to ftp's get utility. It would most likely be called from scripts, rather than directly by a user, as it hides some of the functionality of $\mathrm{ftp}$.

-m machine

-d directory

$-\mathbf{v}$

$-\mathbf{b}$

file
Name or IP address of remote machine to connect to.

Name of directory on remote machine from which to get files. The default directory is the same as that used by ftp.

Verbose. The default is quiet.

Get files as binary. The default is ASCII.

File to transfer.

It attempts to find the user's name for the remote machine by looking for a .netrc file in the user's home directory on the local machine. If no .netrc file is found, the user's name is requested from the terminal. Any necessary password is requested by ftp.

\section{FILES}

$$
\sim / \text { netrc Login information for auto-login }
$$

\section{SEE ALSO}

$$
\text { ftp(1), cmput(L) }
$$

\section{DIAGNOSTICS}

Exit code 0 is returned for success, and exit code 1 is returned for failure.

\section{BUGS}

It is important to note that error messages from ftp may not be trapped, resulting in an exit value of 0 even though an error occurred. 


\section{cmput}

NAME

cmput - a simple interface to ftp.

SYNOPSIS

cmput $-\mathrm{m}$ machine $[-\mathrm{d}$ directory] [-v] [-b] file ...

\section{DESCRIPTION}

cmput provides a simple interface to ftp's put utility. It would most likely be called from scripts, rather than directly by a user, as it hides some of the functionality of $\mathrm{ftp}$.

-m machine

-d directory

$-\mathbf{v}$

$-\mathbf{b}$

file
Name or IP address of remote machine to connect to.

Name of directory on remote machine on which to put files. The default directory is the same as that used by ftp.

Verbose. The default is quiet.

Put files as binary. The default is ASCII.

File to transfer.

It attempts to find the user's name for the remote machine by looking for a .netrc file in the user's home directory on the local machine. If no .netrc file is found, the user's name is requested from the terminal. Any necessary password is requested by $\mathrm{ftp}$.

\section{FILES}

$\sim /$ netre Login information for auto-login

\section{SEE ALSO}

$$
\text { ftp(1), cmget(L) }
$$

\section{DIAGNOSTICS}

Exit code 0 is returned for success, and exit code 1 is returned for failure.

\section{BUGS}

It is important to note that error messages from fitp may not be trapped, resulting in an exit value of 0 even though an error occurred. 


\section{cmlog}

NAME

cmlog - processes cvs log messages.

\section{SYNOPSIS}

cmlog [-T] [-t tag] [-f message file] [-m "message"] [-i] [-h] [-u users] log file

\section{DESCRIPTION}

cmlog may be used in cvs' loginfo file to add a log message to a cvs log file. The available options are:

$-\mathbf{T}$

-t tag

-f message file

-m "message"

$-\mathbf{i}$

$-\mathbf{h}$

-u users

$\log$ file
Add a title to the log file containing the name of the user who did the commit, and a time stamp.

Include a tag in the title, if the - T option is used.

Add the contents of the file message file to the log file.

Add the quoted string "message" to the log file.

Add the standard input to the log file.

Place the log message the top of the log file. The default is to place it at the bottom.

A single user name or a quoted space delimited list of user names to whom a copy of the log entry is sent via Mail.

The absolute pathname of the crs log file.

The text is added in the order shown above-first the title, then the contents of the message file, then the message string, and finally the standard input.

An example of a loginfo file which uses cmlog: 
\# The "loginfo" file is used to control where "cvs commit" log

\# information is sent. The first entry on a line is a regular

\# expression which is tested against the directory that the change

\# is being made to, relative to the \$CVSROOT. If a match is found,

\# then the remainder of the line is a filter program that should

\# expect log information on its standard input.

$\#$

\# The filter program may use one and only one modifier (a la

\# printf). If $\%$ s is specified in the filter program, a brief

\# title is included (enclosed in single quotes) showing the

\# modified file names.

$\#$

\# If the repository name does not match any of the regular

\# expressions in this file, the "DEFAULT" line is used, if it is

\# specified.

\#

DEFAULT cmlog $-i-h$ /codes/ours/logfiles/default.log

$\wedge$ foo1 cmlog $-t-i-h$ /codes/ours/logfiles/foo.log

$\wedge$ foor cmlog $-t-i-h /$ codes/ours/logfiles/foo.log

\section{SEE ALSO}

cvs(1), cvs(5), cmlog_accum(L), cmlog_prep(L)

\section{DIAGNOSTICS}

Exit code 0 is returned for success, and exit code 1 is returned for failure. 


\section{cmlog_accum}

NAME

cmlog_accum - process cvs log messages.

i SYNOPSIS

cmlog_accum [-T] [-h] [-u users $][. ..] \log$ file \%s

DESCRIPTION

cmlog_accum may be used in cvs' loginfo file to add a log message to a cvs log file.

$-\mathbf{T}$

$-\mathbf{h}$

$-\mathbf{u}$ users

log file

$\% \mathrm{~s}$
Add a title to the log file containing the name of the user who did the commit, and a time stamp.

Place the log message at the top of the log file. The default is to place it at the bottom.

A single user name or a quoted space delimited list of user names to whom a copy of the log entry is sent via Mail.

The absolute pathname of the cvs log file.

Signals the cvs loginfo file to include a single-quoted list of modified files. This has to be given in the execute line, or cvs will not include the list of modified files.

This script, when used with cmlog_prep, provides a way to combine individual messages from a multi-directory commit into a single log message. If this is not done, you may see the same log message repeated for every directory involved in a commit. To use cmlog_prep and cmlog_accum, do the following. In the cvs commitinfo file, put in a line to call cmlog_prep. For example:

ALL cmlog_prep

In the loginfo file, put in a line to call cmlog_accum. For example:

ALL Cmlog_accum $-T-h$ /codes/ours/logfiles/foo.log \%s

Now, when you run cave commit, the following happens:

- Just before any files are committed, commitinfo runs cmlog_prep, which creates a file in the / $\mathrm{mp}$ directory. This file lists all directories involved in the commit.

- The files are committed. As they are committed, loginfo runs cmlog_accum to accumulate the individual messages in / $\mathrm{tmp}$. 
- When the last file has been committed, cmlog_accum finishes processing the accumulated messages, and sends them to the permanent log file.

- The files in /tmp are deleted.

SEE ALSO

cvs(1), cvs(5), cmlog_prep(L), cmlog(L)

\section{DIAGNOSTICS}

Exit code 0 is returned for success, and exit code 1 is returned for failure.

\section{BUGS}

The cmlog_accum/cmlog_prep combination does not work when crs import is used. In this case, cvs checks the loginfo file for a program to run, but does not check the commitinfo file. Because of this, the file that cmlog_prep creates does not exist when cmlog_accum looks for it. 


\section{cmlog_prep}

NAME

cmilog_prep - process cvs log messages.

\section{; SYNOPSIS}

cni:og_prep

\section{DESCRIPTION}

cmlog_prep may be used in cvs' loginfo file to add a log message to a cvs log file.

This script, when used with cmlog_accum, provides a way to combine individual messages from a multi-directory commit into a single log message. If this is not done, you may see the same log message repeated for every directory involved in a commit. To use cmlog_prep and cmlog_accum, do the following. In the cvs commitinfo file, put in a line to call cmlog_prep. For example:

$$
\text { ALL cmlog_prep }
$$

In the loginfo file, put in a line to call cmlog_accum. For example:

$$
\text { ALL cmlog_accum }-T-h \text { /codes/ours/logfiles/foo.log \%s }
$$

Now, when you run cave commit, the following happens:

- Just before any files are committed, commitinfo runs cmlog_prep, which creates a file in the /tmp directory. This file lists all directories involved in the commit.

- The files are committed. As they are committed, loginfo runs cmlog_accum to accumulate the individual messages in / $/ \mathrm{mp}$.

- When the last file has been committed, cmlog_accum finishes processing the accumulated messages, and sends them to the permanent log file.

- The files in /tmp are deleted.

\section{SEE ALSO}

$\operatorname{cvs}(1), \operatorname{cvs}(5)$, cmlog_accum(L), cmlog(L)

\section{DIAGNOSTICS}

Exit code 0 is returned for success, and exit code 1 is returned for failure.

\section{BUGS}


The cmlog_accum/cmlog_prep combination does not work when cvs import is used. In this case, cvs checks the loginfo file for a program to run, but does not check the commitinfo file. Because of this, the file that cmlog_prep creates does not exist when cmlog_accum looks for it. 


\section{cmsum}

NAME

cmsum - calculate the checksums of files.

\section{: SYNOPSIS}

cmsum $[-1][-\mathrm{s}$ sum file $][-\mathrm{m}]$ file $[. .$.

\section{DESCRIPTION}

cmsum produces a report on the checksums of files, similar to that produced by the $\operatorname{sum}(1)$ command.

$-1$

-s sum file

$-\mathbf{m}$

file

SEE ALSO

$\operatorname{sum}(1)$

\section{DIAGNOSTICS}

Exit code 0 is returned for success, and exit code 1 is returned for failure.
Local. Do not recursively descend the directory structure. The default is to descend the directory structure.

File containing a list of files and checksums from a previous run of cmsum. It is used to compare with the checksum(s) of the file(s) given by file. The default is to do no comparison.

Flag to require an exact match between the file names in sum file and the file names given in the argument list. This is used to see if any files are missing or added. The default is to compare only the files from the argument list, and to ignore any extra files in sum file. However, it is still an error if a file is given in the argument list and there is no corresponding entry in sum file.

Name of a file or directory to checksum. 


\section{cmvers}

NAME

cmvers - report the value of a tag in a cave tag file.

SYNOPSIS

cmvers [-s separator] tag file

\section{DESCRIPTION}

cmvers reports the value of a tag in a cave tag file.

-s separator

When reporting, replace the field separator as given in the tag file with separator. The tag file itself is not changed.

tag file

Name of the tag file.

cvs restricts the characters that may be used in tags-they may not contain a semicolon, colon, dollar sign, at sign, comma or period (;:\$@,.). The -s argument is supplied so that the user may replace the tag's field separator with one of these forbidden characters and use the tag for some other purpose, such as a version number. For example, if the tag file named a.tag contained the following:

$$
\text { project V_002_a0 - fixed }
$$

Then,

$$
\text { cmvers a.tag }
$$

returns the value

$$
\text { V_002_a0 }
$$

But

$$
\text { cmvers -s a.tag }
$$

returns the value

$$
\text { V.002.a0 }
$$

SEE ALSO

$$
\text { cave(L), } \operatorname{cvs}(1), \operatorname{cvs}(5)
$$

\section{DIAGNOSTICS}

Exit code 0 is returned for success, and exit code 1 is returned for failure. 


\section{Project Description File}

\section{File Format}

A project description file is a text file which describes the root directories, source repositories, subdirectories and locks used by a project. It contains the information needed to customize the operation of the cave tool. The CAVE_PD environment variable may be set to the full path name of the project description file. Note that you do not need to set the CVSROOT environment variable yourself in order to use cave.

$\therefore$ : nile may contain blank lines. The symbol \# is the comment symbol. Everything after it on a line is ignored. Long lines may be continued to the next line by typing a backslash (V) first.

All other lines must start with a keyword. Keyword lines which apply to the entire project may be in any order (i.e. group and access).

Lines which come after a root_dir line apply to the specified root directory, until a root_dir end line.

Lines which come after a repository line apply to the specified root directory, until a repository end line. 
Please note that each root directory is assumed to contain the cvs administrative directory called CVSROOT. This does not need to be specified in the project description file.
project project_name
(Required)
group group_name
(Required)
access [group] user_name [...]
(Required)
mail [access] user_name [...]
(Optional)
lock lock_dir [lock_type...]
(Optional)
login_grp yes I no
(Optional)

pack distribution [[path |-x other ]...] (Optional. Repeat as needed.)

print lpr_line

(Optional. Repeat as needed.)

precheckout prog $[$ arg Is arg ...]

(Optional. Repeat as needed.)

postcheckout prog [arg Is_arg ...]

(Optional. Repeat as needed.)

precommit prog $\left[\right.$ arg $\mid s_{-}$arg ...]

(Optional. Repeat as needed.)

postcommit prog $\left[\right.$ arg $\left.\mid s \_a r g . ..\right]$

(Optional. Repeat as needed.)

reserve reserve_dir

(Optional)

tag tag file [module ...]

(Optional.)

root dir name

(Must te one root dir name line for each root directory in the project.)

repository name

(Must be one repository name line for each source repository in the preceding root_dir.)

repository end

(Must be one repository end line for each repository name line.)

[Repeat repository keyword line as needed.]

root_dir end

(Must be one root_dir end line for each root_dir name line.).

[Repeat root_dir keyword line as needed.] 


\section{Keywords}

access [group] user_name [...]

group

All members of the group_name given with the group keyword.

user_name Name of user.

Optional: no

Repeatable: no

Applies to: entire project

Determines who has access to the project. The access list is the union of the group members (if requested) and the list of user names.

It is important to note that access to the directories and files is controlled by the Unix file permissions (rwx). The only thing the access does is to add some error checking which has proven to be useful with beginners working on multiple projects.

This information is used by most of the cave commands.

group group_name

group_name Name of the group for the project.

Optional: no

Repeatable: no

Applies to: entire project

This determines group ownership for the repository, and any new files.

This information is used by most of the cave commands. 
lock

$$
\text { lock_dir [lock_type ...] }
$$

lock_dir

lock_type
Absolute pathname of a directory to hold the lock files.

Type of lock to be used with this project, The default is to use all available types.

Optional: yes

Repeatable: yes

i Applies to: entire project

The available types of locks are:

extract

Lock name: extract.project.pid

Created by: checkout, diff, export, history, pack, print, status, update

When:

Extracting information or files from the repository.

Checked by: All cave commands that change the repository.

Note:

This lock should not be touched by the user under normal circumstances. More than one extract lock may be present at a time.

insert Lock name: insert.project

Created by: add, commit, release, rtag, tag

When: Changing the repository.

Checked by: All cave commands that use information from the repository.

Note: $\quad$ This lock should not be touched by the user under normal circumstances.

panic

Lock name: panic.project

Created by: user

When: A panic situation arises

Checked by: All cave commands that use information from the repository.

Note: $\quad$ To be used for emergencies only.

update Lock name: update.project

Created by: user

When: $\quad$ A general lock of the project is needed while doing several operations, which may not be under cave's control.

Checked by: All cave commands that use information from the repository.

Note: $\quad$ A general purpose lock.

If a particular type of lock is not defined by the lock line in the project cescription file, any commands which check for that type of lock assume that none is required. 
login_grp yes I no

Optional: yes

Repeatable: no

Applies to: entire project

If this command is no: used, cave assume that login_grp is set to "yes".

When login_grp is "yes", cave checks that the user is logged in under the correct group when running any cave command that changes the repository. If the user is not, cave stops and advises that newgrp be run. Note that this is not the same as checking if the user is a member of the correct group.

The reason for checking the current group of a user is that cvs commits files under the group that the user is logged in under. It ignores what the file's group id is in the user's local workspace and in the repository.

If you wish to avoid this check, and still ensure that files maintain the correct group id, run the shell command:

$$
\text { chmod }-\mathrm{R} \text { g+s root_dir }
$$

on each root directory in the project. This forces any committed files to be the correct group.

This information is used by the cave command commit, lock, reserve, rtag, tag and unreserve..

mail

$$
\text { [access] user_name [...] }
$$

access

Flag to send mail to everyone with access to the root directory, as defined by the access keyword line. If used, this flag must be before any user name.

user name

Name(s) of additional user(s) to receive mail.

Optional: yes

Repeatable: no

Applies to: entire project

If the mail keyword is used, mail is sent to everyone on the list whenever one or more files are committed to this project.

This information is used by the cave command commit. 


\section{pack} distribution

-x other

path distribution [[path |-x other ]...]

Name to assign to this distribution.

The name of a directory or file which is within one of the root directories, yet not under cave control. It must start with the tail of a root directory pathname, and must be followed by the relative pathname of a file or subdirectory. If other ends with a directory name, all files contained within that directory are included.

Note: These files are always collected from the root directory. The "-w from" argument in cave pack has no effect.

This is a pathname which must start with the tail of a root directory pathname, and may be followed by the relative pathname of a file or subdirectory under cave control. If path ends with a directory name, all files contained within that directory are included. The subdirectory and file names must be the names as they exist in the repository (without the ",v" suffix), not the names of keys defined in the modules file.

Optional: yes

Repeatable: yes

Applies to: entire project

If the pack key line is used, and no path or -x other is given, the default is to include all of the files in the project which are under cave control.

This information is used by the cave command pack.

postcheckout prog $\left[\arg \mid s_{-} \arg \ldots\right]$
prog
Full pathname of program or script to run as part of the checkout command, right after checking out files from the repository.
arg
s_arg
Argument to prog.
A symbol for cave to substitute a string containing information about which root directories and files were requested by the user. Any default values are filled in. The symbols and their resulting space delimited strings are:
$\%$ She full path name of each root directory requested for the checkout. Each root directory is followed by a list of requested directories and/or files. The pathnames of the directories and files are relative to the root directory. 
\%osp The pathnames, relative to the root directory, of the directories and files requested for the checkout.

\%sr The full path name of each root directory requested for the checkout.

Optional: yes

Repeatable: yes

Applies to: entire project

Each postcheckout program is run in sequence whenever the checkout command is used. Any program which exits with a non-zero status is assumed to have failed, and cave ends with an error exit. See the description of the checkout command for information on exactly when the postcheckout programs are run in relation to other programs used during the checkout process.

Multiple spaces in the postcheckout line are compressed to one space.

Suggestion: If prog is your own script or executable, make any messages it sencs to the terminal distinct by prepending the name prog to the message. Without this, it can be difficult to tell what is responsible for a message. 
postcommit prog $\left[\arg \mid s_{-} a r g \ldots\right]$

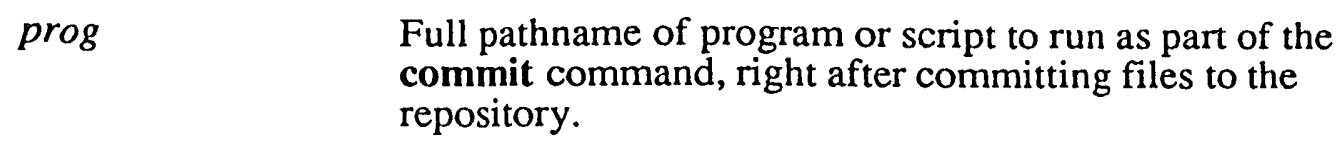

arg Argument to prog.

s_arg A symbol for cave to substitute a string containing information about which root directories and files were requested by the user. Any default values are filled in. The symbols and their resulting space delimited strings are:

\%s The full path name of each root directory requested for the commit. Each root directory is followed by a list of requested directories and/or files. The pathnames of the directories and files are relative to the project directory.

\%sp The pathnames, relative to the project directory, of the directories and files requested for the commit.

\%sr The full path name of each root directory requested for the commit.

Optional: yes

Repeatable: yes

Applies to: entire project

Each postcommit program is run in sequence whenever the commit command is used. Any program which exits with a non-zero status is assumed to have failed, and cave ends with an error exit. See the description of the commit command for information on exactly when the postcommit programs are run in relation to other programs used during the commit process.

This is different than what is done with cvs' loginfo file, which keys off of module names in determining which programs to run after committing files

Multiple spaces in the postcommit line are compressed to one space.

Suggestion: If prog is your own script or executable, make any messages it sends to the terminal distinct by prepending the name prog to the message. Without this, it can be difficult to tell what is responsible for a message. 
precheckout prog $\left[\arg \mid s_{-}\right.$arg .... $]$
prog
Full pathname of program or script to run as part of the checkout command, right before checking out files from the repository.
$\arg$
s_arg
Argument to prog.
A symbol for cave to substitute a string containing information about which root directories and files were requested by the user. Any default values are filled in. The symbols and their resulting space delimited strings are:

\%s The full path name of each root directory requested for the checkout. Each root directory is followed by a list of requested directories and/or files. The pathnames of the directories and files are relative to the root directory.

\%sp The pathnames, relative to the root directory, of the directories and files requested for the checkout.

\%sr The full path name of each root directory requested for the checkout.

\author{
Optional: yes \\ Repeatable: yes \\ Applies to: entire project
}

Each precheckout program is run in sequence whenever the checkout command is used. Any program which exits with a non-zero status is assumed to have failed, and cave ends with an error exit. See the description of the checkout command for information on exactly when the precheckout programs are run in relation to other programs used during the checkout process.

Multiple spaces in the precheckout line are compressed to one space.

Suggestion: If prog is your own script or executable, make any messages it sends to the terminal distinct by prepending the name prog to the message. Without this, it can be difficult to tell what is responsible for a message. 


\section{precommit prog $\left[\right.$ arg $\left.\mid s \_a r g \ldots\right]$}
prog Full pathname of program or script to run as part of the commit command, right before committing files to the repository.

arg Argument to prog.

s_arg A symbol for cave to substitute a string containing information about which root directories and files were requested by the user. Any default values are filled in. The symbols and their resulting space delimited strings are:

\%s The full path name of each root directory requested for the commit. Each root directory is followed by a list of requested directories and/or files. The pathnames of the directories and files are relative to the project directory.

\%sp The pathnames, relative to the project directory, of the directories and files requested for the commit.

\%sr The full path name of each root directory requested for the commit.

Optional: yes

Repeatable: yes

Applies to: entire project

Each precommit program is run in sequence whenever the commit command is used. Any program which exits with a non-zero status is assumed to have failed, and cave ends with an error exit. See the description of the commit command for information on exactly when the precommit programs are run in relation to other programs used during the commit process.

This is different than what is done with cv's' commitinfo file, which keys off of module names in determining which programs to run before commiting files.

Multiple spaces in the precommit line are compressed to one space.

Suggestion: If prog is your own script or executable, make any messages it sends to the terminal distinct by prepending the name prog to the message. Without this, it can be difficult to tell what is responsible for a message. 
print lpr_line

lpr_line Line to be used with the Ipr print spooler.

Optional: yes

Repeatable: yes

Applies to: entire project

Multiple print lines are allowed, so that each user's output command may be customized.

This information is used by the cave command print.

project project_name

project_name Name of the project.

Optional: no

Repeatable: no

Applies to: entire project

This information is used by most of the cave commands.

reserve reserve_dir

reserve_dir Absolute pathname of a directory to hold information about reserved directories and files. This directory should not be used for any other purpose.

Optional: yes

Repeatable: no

Applies to: entire project

The presence of this line in the project description file causes cave to check for reserved directories and files before allowing the checkout and commit operations. It allows the reserve and unreserve commands to be used. 


$$
\begin{gathered}
\text { root_dir } \\
\text { root_dir } \\
\text { name }
\end{gathered}
$$

Absolute pathname of the cvs root directory. This is the value to which the environment variable CVSROOT is set. You do not need to set CVSROOT yourself in order to use cave.

Optional: no

Repeatable: yes

Applies to: entire project

There must be one root_dir name line for each root directory in the project. Lines which come after a root_dir name line apply to the specified root directory, until the next root_dir end line.

This information is used by most of the cave commands.

\section{repository name \\ repository end}

name
Name of the source repository, relative to the root directory.

Optional: no

Repeatable: yes

Applies to: root directory

There must be one repository name line for each source repository in the project. Lines which come after a repository name line apply to the specified source repository, until the next repository end line. Please note that each root directory is assumed to contain the cvs administrative directory called CVSROOT. This does not need to be specified in the project description file.

This information is used by most of the cave commands. 
tag tag file $[$ module ...]

tag_file

module
Absolute pathname to a tag file containing information about the tag to use when committing files.

A key, as defined in the modules file.

If a project uses more than one root directory, the module must start with the tail of the absolute root directory pathname and continue with the module, as defined in the modules file:

\section{root dir/module}

These keys determine what files to tag. If the tag key line is used, and no module is given, the default is to use the names of all the source repositories in this project.

$\begin{array}{ll}\text { Optional: } & \text { yes } \\ \text { Repeatable: } & \text { no } \\ \text { Applies to: } & \text { entire project }\end{array}$

This insures that all relevant files get tagged, even if they were not directly involved with a commit. If no tag key line is used, no files are tagged.

The tag file is a text file. See the chapter "Tag File" for a description of the correct format for a tag file.

This information is used by the cave command commit. 


\section{Tag File}

The tag file is a text file which contains the value of the last tag which was used for the project. The file is referenced in the project description file, and is used during a commit. It may contain any number of lines, but the first line must be in the following format:

project tag_value [field_separator [fixed I variable]]

The values in this line are:
tag_value
The current value of the tag. The tag must start with a letter, and must not contain a semicolon, colon, dollar sign, at sign, comma or period (;:\$@,.).
field_separator
Field separator for the tag. The default is to use an underscore ( ).

fixed I variable

This tells if the field lengths should remain constant. The default is to use variable.

Any fields which might be incremented may contain any combination of digits and letters. For example, if the first line of the file looks like this before a commit:

$$
\text { project V_001_a9 _ fixed }
$$

It would lock like this after a commit:

$$
\text { project V_001_b0 - fixed }
$$

The default is to increment the rightmost field by one. Note that for the fixed field mode, the number of characters in each field remained constant. If a field reached its maximum value, the next field gets incremented:

$$
\text { project V_001_z9 - fixed }
$$

becomes:

$$
\text { project V_002_a0 - fixed }
$$

In contrast, a tag with a variable fiela might look like this before it is incremented:

$$
\text { project V_1_9 }
$$

and like this after:

project V_1_10 


\section{CVS Commands and Related Files}

\section{General Information}

Read the cvs(1) man page for a complete description of the cvs command and its options.

The execute line for cvs is:

cvs [cvs_options] cvs_command [command_options] [command_args]

The cvs command line can include cvs_options, which apply to the overall cvs program; a cvs_command, which specifies a particular action on the source repository; and command_options and command_args to fully specify what the cvs_command will do.

Warning: you must be careful of precisely where you place options relative to the cvs_command. The same option can mean different things depending on whether it is in the cvs_options position (to the left of a cvs command) or in the command_options position (to the right of a cvs command).

There are only two situations where you may omit cvs_command: "cvs -H" elicits a list of available commands, and "cvs - $v$ " displays version information on cvs itself.

The cvs_options control the overall cvs program:

$-\mathrm{H}$

$-\mathbf{Q}$

$-\mathbf{q}$

-b bindir
Display usage information about the specified cvs_command (but do not actually execute the command). If you don't specify a command name, "cvs - H" displays a summary of all the commands available.

Causes the command to be really quiet; the command will generate output only for serious problems.

Causes the command to be somewhat quiet; informational messages, such as reports of recursion through subdirectories, are suppressed.

Use bindir as the directory where rcs programs are located. Overrides the setting of the RCSBIN environment variable.

This value should be specified as an absolute pathname.

-d CVS_root_directory Use CVS_root_directory as the root directory pathname of the master res source repository. Overrides the 


\begin{tabular}{|c|c|}
\hline -e editor & $\begin{array}{l}\text { Use editor to enter revision log information. Overrides the } \\
\text { setting of the EDITOR environment variable. }\end{array}$ \\
\hline$-I$ & $\begin{array}{l}\text { Do not log the cvs command in the command history (but } \\
\text { execute it anyway). See the description of the history } \\
\text { command for information on command history. }\end{array}$ \\
\hline$-\mathbf{n}$ & $\begin{array}{l}\text { Do not change any files. Attempt to execute the } \\
\text { cvs command, but only to issue reports; do not remove, } \\
\text { update, or merge any existing files, or create any new files. }\end{array}$ \\
\hline$-t$ & $\begin{array}{l}\text { Trace program execution; display messages showing the } \\
\text { steps of cvs activity. Particularly useful with }-n \text { to explore } \\
\text { the potential impact of an unfamiliar command. }\end{array}$ \\
\hline$-\mathbf{r}$ & $\begin{array}{l}\text { Makes new working files read-only. Same effect as if the } \\
\text { CVSREAD environment variable is set. }\end{array}$ \\
\hline$-\mathbf{v}$ & Displays version and copyright information for cvs. \\
\hline$-w$ & $\begin{array}{l}\text { Makes new working files read-write (default). Overrides } \\
\text { the setting of the CVSR:EAD environment variable. }\end{array}$ \\
\hline
\end{tabular}

setting of the CVSROOT environment variable. This value should be specified as an absolute pathname.

Use editor to enter revision log information. Overrides the setting of the EDITOR environment variable. execute it anyway). See the description of the history

Do not change any files. Attempt to execute the cus command, but only to issue reports; do not remove, Trace program execution; display messages showing the steps of cvs activity. Particularly useful with $-n$ to explore the setting of the CVSR:EAD environment variable. 


\section{Commands}

There are equivalent cave commands for most of the cvs commands. The following commands, however, must be run directly from cvs.

admin [rcs-options] files ...

Requires: repository, working directory

Changes: repository

Synonym res

This is the cvs interface to assorted administrative res facilities, documented in res(1). 'cvs admin' simply passes all its options and arguments to the res command; it does no filtering or other processing. This command does work recursively, however, so extreme care should be used.

import [-options] repository vendortag releasetag ...

Requires: repository, source distribution directory

Changes: repository

Use this command to incorporate an entire source distribution from an outside source into your root directory. You can use this cornmand both for initial creation of a repository, and for wholesale updates to the module from an outside source.

The repository name gives a directory name under the cvs root directory. If the directory does not exist, import creates it. The vendortag and releasetag arguments are tags to identify the initial source and to distinguish it from subsequent imports. 
rdiff [-flags] [-V vn] [-r $t \mid-\mathbf{D} d[-\mathbf{r} t 2 \mid-\mathbf{D} d 2]]$ modules...

Requires: repository

Changes: nothing

Synonym patch

Builds a Larry Wall format patch(1) file between two releases, that can be fed directly into the patch program to bring an old release up-to-date with the new release. (This is one of the few cvs commands that operates directly from the repository, and doesn't require a prior checkout.) The diff output is sent to the standard output device. You can specify (using the standard -r and -D options) any combination of one or two revisions or dates. If only one revision or date is specified, the patch file reflects differences between that revision or date and the current "head" revisions in the res file.

Note that if the software release affected is contained in more than one directory, then it may be necessary to specify the -p option to the patch command when patching the old sources, so that patch is able to find the files that are located in other directories.

If you use the option $-\mathrm{V} v n$, rcs keywords are expanded according to the rules current in res version $v n$ (the expansion format changed with res version 5).

The standard option flags $-\mathbf{f},-\mathbf{l},-\mathbf{Q}$, and $-\mathbf{q}$ are available with this command. There are also several special options flags:

-s No patch output is produced. Instead, a summary of the changed or added files between the two releases is sent to the standard output device. This is useful for finding out, for example, which files have changed between two dates or revisions.

$-t \quad$ A diff of the top two revisions is sent to the standard output device. This is most useful for seeing what the last change to a file was.

-u The parch output uses the newer "unidiff" format for context diffs.

-c Explicitly specify the "diff -c" form of context diffs (which is the default). 


\section{Support Files}

cvs has several support files. None of them are necessary for cvs to run, however they allow the user to customize the operation of the system. The files exist in the directory \$CVSROOT/CVSROOT, where \$CVSROOT is the pathname to the root directory which hold the repositories. The files are:

\section{\$CVSROOT/CVSROOT/modules, $v$ \\ \$CVSROOT/CVSROOT/commitinfo,v \\ \$CVSROOT/CVSROOT/loginfo,v \\ \$CVSROOT/CVSROOT/resinfo,v \\ \$CVSROOT/CVSROOT/editinfo,v \\ \$CVSROOT/CVSROOT/cvsignore, $v$ \\ \$CVSROOT/CVSROOT/history}

Here are short descriptions of the files. For more devailed information, read the cvs(5) man page.

modules

Define symbolic names or aliases for files or' groups of files.

commitinfo

Define programs to run just before running cvs commit.

loginfo

Define programs to run after cvs commit, to send log messages to a fille.

resinfo

Define forms for the log messages.

editinfo

Define programs to validate the log messages.

cvsignore

Specify a list of files to ignore during cvs update. 


\section{history}

A file which holds a record of cvs commands which affect the repository, such as commit or tag. It can be read with the cvs history command. 


\section{Support Programs}

cvsinit

A shell script that can be used to set up an initial \$CVSROOT/CVSROOT directory, where \$CVSROOT is the pathname to the root directory which holds the repositories. This only needs to be done once, at the time a cvs root directory is being created. The files created by cvsinii ${ }^{1}$ are described earlier in this chapter, under Support Files. These files need to be edited, to customize them for a particular root directory.

mkmodules directory

Rebuilds the modules database that $\operatorname{cvs}(1)$ uses whenever the modules or loginfo files are changed. The directory is expected to contain the modules, $v$ and loginfo,v files. For more detailed information, read the mkmodules(1) man page.

${ }^{1}$ Our version of evsinit on the Peregrine machine at LLNL has a small modification which allows it to be run from any directory. If you are using the unmodified version, you must find the directory that contains the source distribution for cvs. It is probably located somewhere in /usr/local. On our machine, for example, it is in $/ \mathrm{usr} / \mathrm{local} / \mathrm{src} / \mathrm{gnu} / \mathrm{cvs} / \mathrm{vl}$.3. This directory should contain the script called crsinit. It must be run from within the cvs source directory (i.e. /usr/local/src/gnu/cvs/v1.3). 


\section{Appendix A: Installing CAVEMAN}

CA VEMAN is installed and maintained at LLNL:

Closed Labnet $\quad$ Peregrine (130.106.66.1), an A Division machine.

Open Labnet Contact the author.

The tools needed to install it on other platforms are listed below.

It is comprised of several scripts which must be preprocessed. The scripts and a make file to do the preprocessing are available on Peregrine in /codes/CAVEMAN_take.

The CAVEMAN system uses several free software programs that are available via anonymous ftp from any one of several UNDX archives. Possible sources are:

$\begin{array}{ll}\text { prep.ai.mit.edu } & 18.71 .0 .38 \\ \text { uunet.uu.net } & 192.48 .96 .2 \\ \text { tut.cis.ohio-state.edu } & 128.146 .8 .60 \\ \text { jpl-devvax.jpl.nasa.gov } & 128.149 .1 .143\end{array}$

The programs are available from the Free Software Foundation under the terms of the GNU General Public License or the Artistic License. The conditions of use of the programs are included with the source code. The user is responsible for following any requirements, restrictions and rules.

Each program comes with documentation, including man pages and README files.

The programs required by CAVEMAN are: 


\begin{tabular}{|l|l|l|}
\hline Program & Version & Notes \\
\hline rcs & 5.6 & $\begin{array}{l}\text { Revision Control System. } \\
\text { Automates the storage, retrieval, logging, identification } \\
\text { and merging of source revisions. } \\
\text { It was originally designed and built by Walter F. Tichy } \\
\text { of Purdue University. }\end{array}$ \\
\hline diff3 & 1.15 (or later) & $\begin{array}{l}\text { Shows the differences between files. } \\
\text { GNU DIFF was written by Mike Haertel, David Hayes, } \\
\text { Richard Stallman and Len Tower. }\end{array}$ \\
\hline cvs & 1.3 & $\begin{array}{l}\text { Concurrent Versions System. } \\
\text { cvs is a front end to the rcs control system. } \\
\text { Several people have contributed to cvs. The two main } \\
\text { authors are Brian Berliner of Sun Microsystems, Inc. and } \\
\text { Jeff Polk of BSDI. }\end{array}$ \\
\hline perl & $\begin{array}{l}4.0 .1 .4 \\
\text { Patch level 10 }\end{array}$ & $\begin{array}{l}\text { perl is a scripting language for manipulating text, files } \\
\text { and processes. } \\
\text { It was written by Larry Wall of JPL. }\end{array}$ \\
\hline
\end{tabular}




\section{Appendix B: Tutorial}

The CAVEMAN system is a front end to cvs (Concurrent Versions System), which is in turn a front end to the res revision control system.

It maintains files in directories called repositories. The repositories are contained in a directory called a root directory. In addition to the repositories, this root directory contains a special directory named CVSROOT which holds several special files used by cvs for administrative purposes. The root directory may also contain any other files and directories you care to put in it, such as $\log$ files and project description files.

The files in the repositories should never be changed directly. The files are "checked out" into the user's local workspace, where any changes are made. The modified files are then "committed" back into the repository. More than one person may check out the same files at the same time. Any conflicts are caught by cvs at commit time.

cvs keeps track of all changes, so that older revisions of the files may also be checked out.

The main tool in CAVEMAN is named cave. It has several commands. The only ones you need to actually change files in a repository are:

cave checkout path ...

Creates your private copy of all or part of the files in the repository.

cave update

Updates your private copies of the files with changes made to the repository since the files were checked out.

cave add file...

Marks new files to be added to an existing repository. They are not actually added until cave commit is run.

cave remove file...

Marks files to be deleted from an existing repository. They are not actually removed until cave commit is run.

cave commit path ...

Incorporates your changes into the repository. 
Read the chapter "Assumptions and Restrictions". It is located in the early part of this document.

I repeat, read the chapter "Assumptions and Restrictions". It also won't hurt to read the cvs man pages.

There is a glossary at the end of this document which defines some of the common terms. 


\section{Pictorial Overview of a Project}

Let us look at a project named ours. It has the following parts:

- A project description file named /codes/our_sources/OurPD.

- A root directory named/codes/our_sources which contains two repositories-foo and fum.

- A root directory named /stuff/other_sources which contains two repositories-boo and bar.

- A single log file named/codes/our_sources/related/our.log.

- A tag file named/codes/our_sources/related/tag.file.

The layout of the directories is :
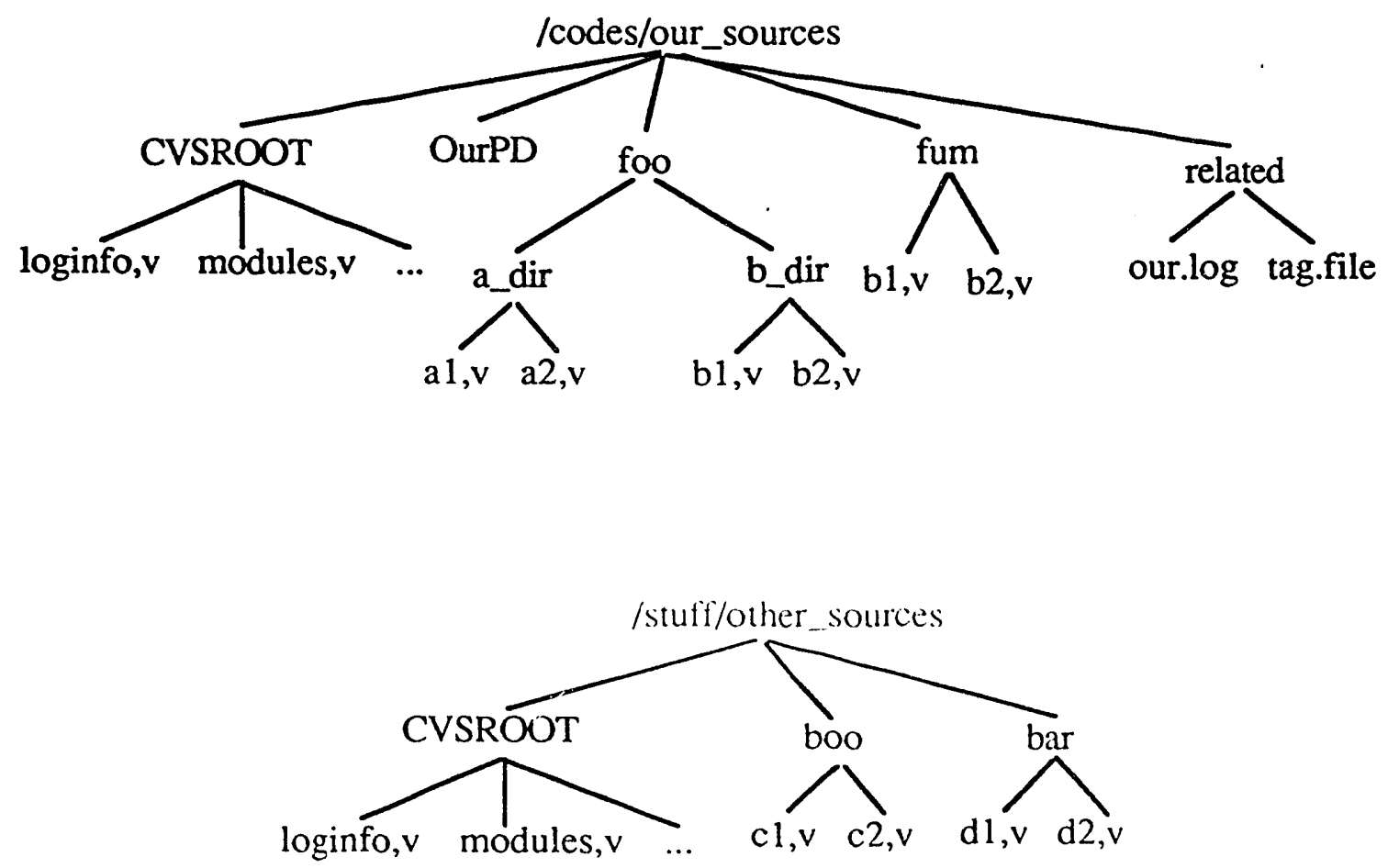

The project description file for this example looks like:

\# Project description file for the OURS project.

$\begin{array}{ll}\text { project } & \text { ours } \\ \text { group } & \text { our_group }\end{array}$ 


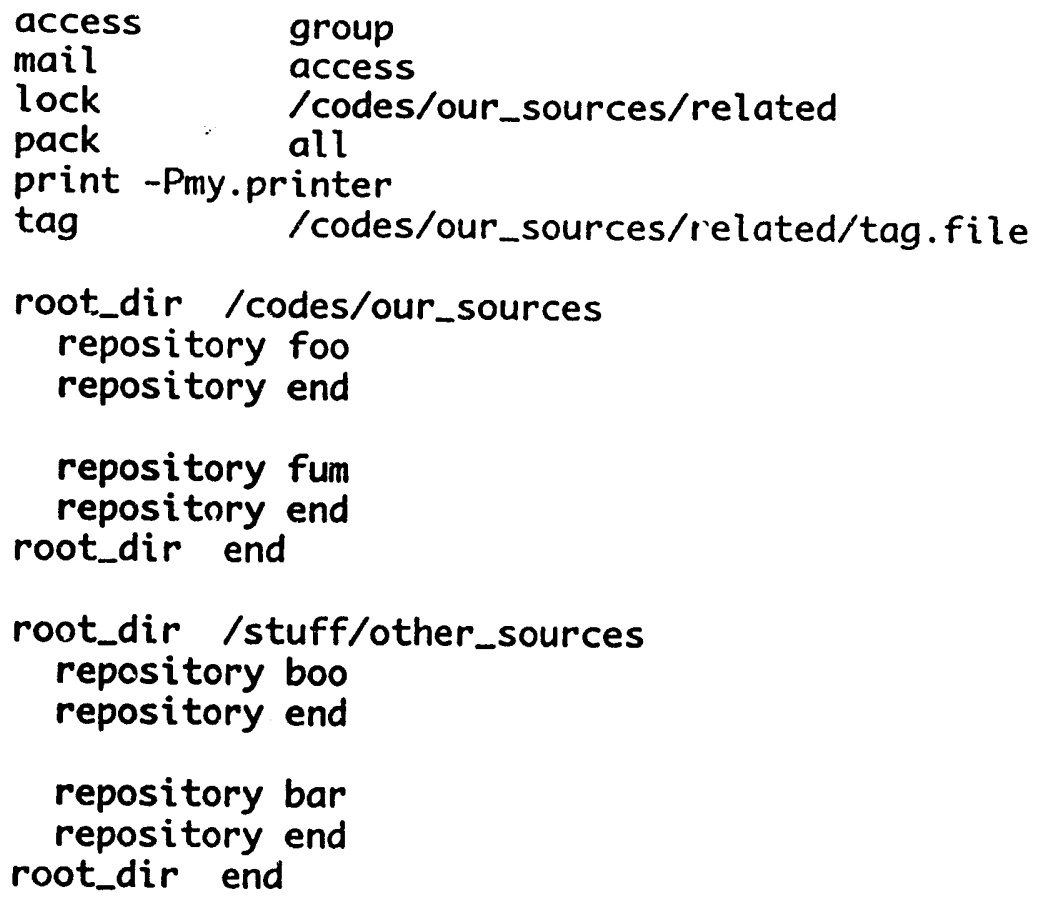




\section{Installing the Initial Sources}

A root directory is a directory that contains cvs repositories and a subdirectory named CVSROOT which contains several cvs administration files.

Let us assume you wish to create a new root directory called/codes/our_sources and have it contain one repository named foo. The reposi:ory is to contain two subdirectories named a_dir and b_dir.

As you go through the procedure, make sure that any files and directories you create have the correct group id and access controls for your purposes.

Note: To make these instructions easier to follow, several of the messages sent by crs are eliminated in this examiple.

First, set the environment variable CVSROOT to the name of your new root directory. This needs to be set before running cvs.

$$
\text { setenv CVSROOT /codes/our_sources }
$$

Run cvsinit ${ }^{2}$ to create the root directory and set up the administration files.

$$
\text { cvsinit }
$$

Move to the new root directory. Put in directories for anything else you may want to store here, such as the project description file, tag files, log files or scrifts. These files may be stored in any directory, but it is easier to find them if they are within the root directory.

Assume the log file is to be named our.log, and is to be placed in a directory named related.

$$
\begin{aligned}
& \text { cd /codes/our_sources } \\
& \text { mkdir related }
\end{aligned}
$$

You may wish to set up a header for your log file, at this time, or at least create an empty log file:

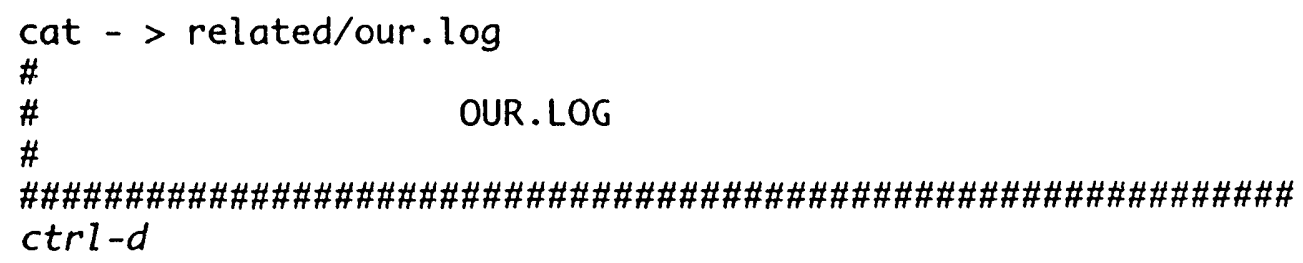

\footnotetext{
2Our version of evsinit on the Peregrine machine at LLNL has a small modification which allows it to be run from any directory. If you are using the unmodified version, you must find the directory that contains the source distribution for cvs. It is probably located somewhere in /usr/local. On our machine, for example, it is in $/$ usr/local/sre/gnu/cvs/v1.3. This directory should contain the script called cvsinit. It must be run from within the cvs source directory (i.c. /usr/local/src/gnu/cvs/v1.3).
} 
Assume the tag file is to be named tag.file, and is also to be placed in related:

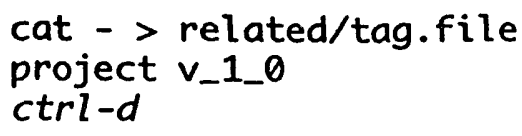

Create a project description file as defined earlier in this document. Let us call it OurPD, and place it in /codes/our_sources. The tag file and project description file may be stored in any directory, but it is easier for people to find them if they are within the root directory.

The project description file for this example looks like this:

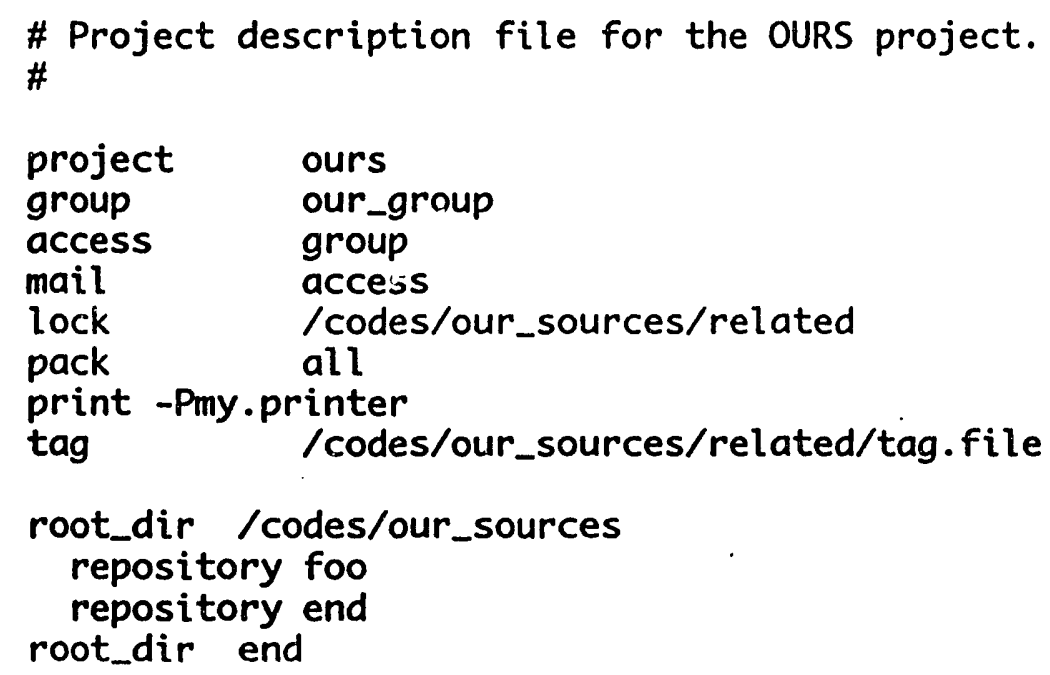

Now we need to edit the administrative files, such as modules and loginfo. These were created by cvsinit. Also, samples of these files come with crs. If you know where the crs source directory is on your machine, look in a subdirectory called examples.

Move to some directory not contained in /codes/our_sources. Let us assume it is called /home/me/work. Check out CVSROOT.
cd /home/me/work
cVs checkout CVSROOT
cd CVSROOT

The directory structure should now look like this: 


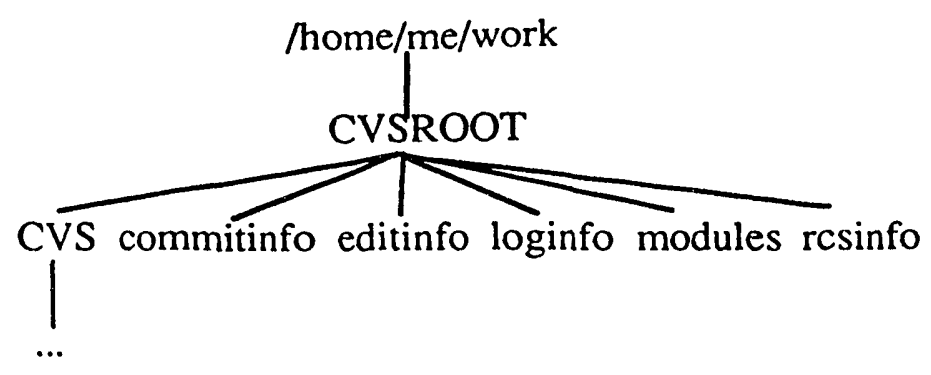

Edit any of the files as needed.

The loginfo file can use any sort of filter program, such as cat, to send output to a log file. Here is an example that uses a local script called cmlog:

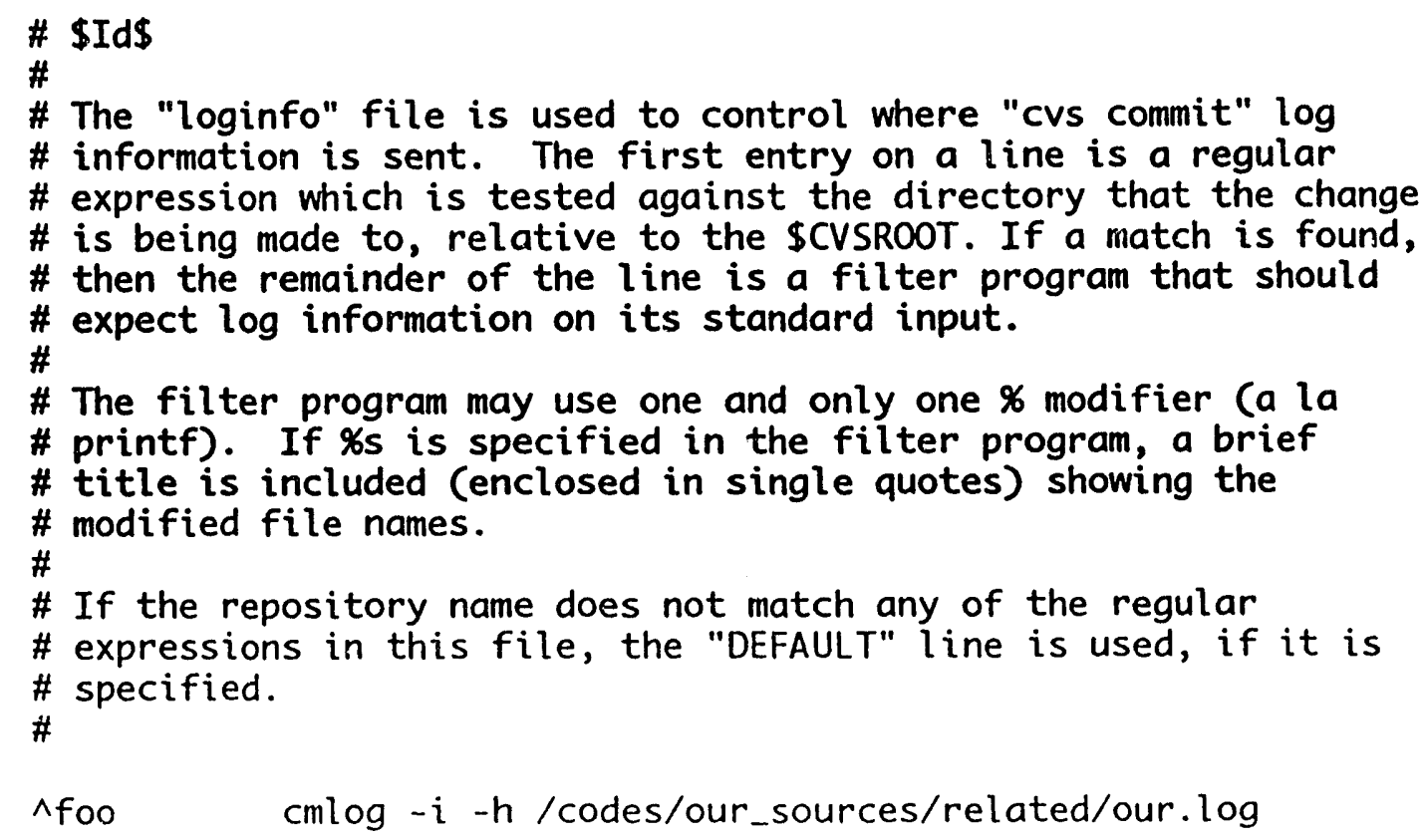

The modules file should look something like this: 


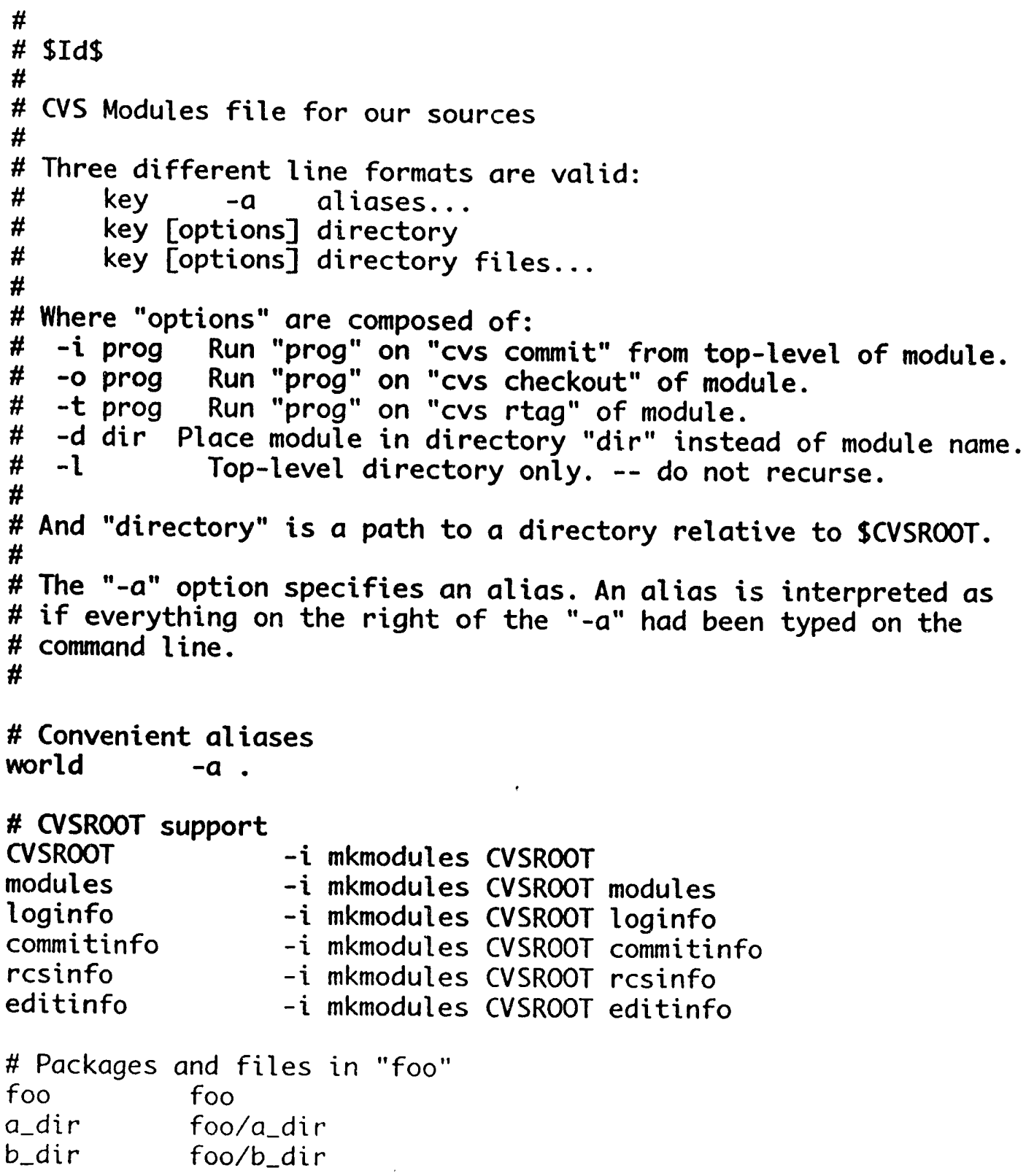

For more information on how to set up a modules file for CAVEMAN, see "Appendix C: The Modules File".

You may want to create a cvsignore file, which is used to specify a list of files to ignore during update. I recommend creating this file, even if it only contains a blank line. If you don't, cvs tells you at almost every opportunity how nice it would be to have a cvsignore file.

Create the cvsignore file in the /home/me/work/CVSROOT directory. Then type the following:

cvs add -m "New cvsignore file." cvsignore 
Now, commit all the changes:

cVs commit -m "Initial changes to CVSROOT files."

You may now remove the CVSROOT directory in /home/me/work.

The source needs to be prepared. Put the source in some directory not contained in our sources. Let us assume it is /home/me/work. Divide the source into directories and files, with the same hierarchy as you want them maintained by cvs.

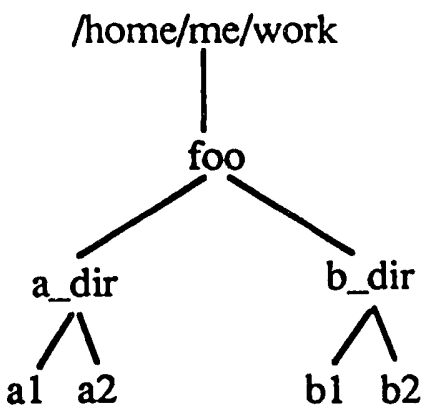

Optional: Put a comment line containing "\$Id\$" at or near the iup of each file. cvs then places information about the version number and date into the comment line whenever the file is checked in. Suppose you insert a line that looks like this in file a1:

\section{c \$Id\$}

After the file is placed under cave control, the line looks something like this:

$$
\text { c \$Id: a1,v } 1.01992 / 02 / 02 \text { 13:53:36 me Exp \$ }
$$

Optional: Put a comment line containing "\$Log\$" at or near the top of each file. crs puts a log message at this location each time the file is committed. The log messages are cumulative.

The process of adding the \$Id $\$$ and $\$ \log \$$ can be automated by using cave header.

Move to /home/me/work/foo, the top level directory of your source. To install the source, with a tag of "FOO" and a version number of "v_1_0", type:

cvs import $-m$ 'Initial installation of foo.' foo FOO v_1_0

The directory/codes/our_sources/foo should now contain files that have the same names as the source files, with a ",v" appended. The root directory should look like: 


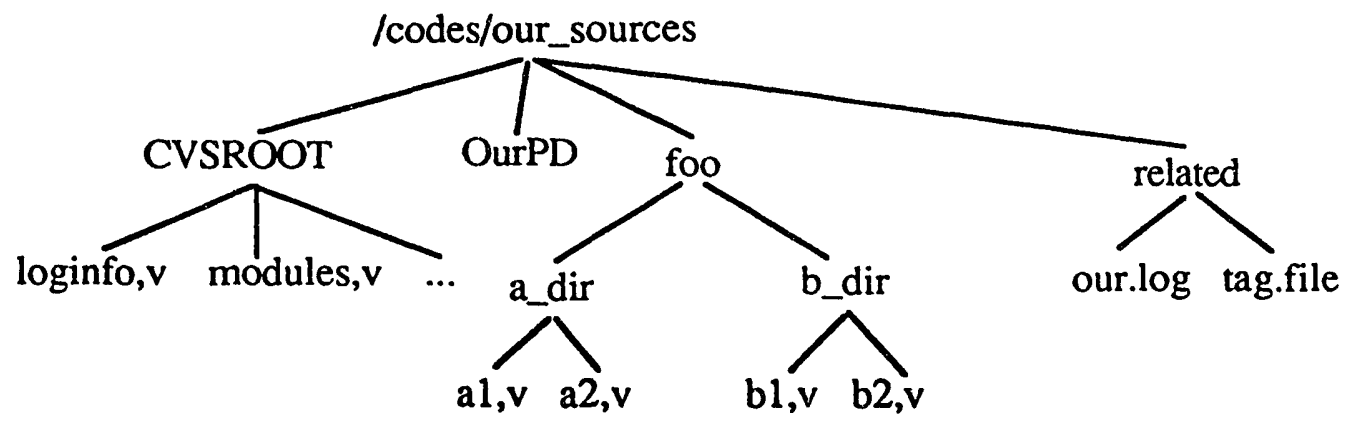

Make sure that any files and directories you created have the correct group id and access controls for your purposes. You may want to use the shell command:

$$
\text { chmod }-R \text { g+s /codes/our_sources }
$$

This forces any committed files to be the correct group. This ensures that any files committed in the future will have the correct group in the repository.

You may now remove the files in /home/me/work/foo. 


\section{Adding a Second Root Directory to a Project}

A root directory is a directory that contains cvs repositories and a subdirectory named CVSROOT which contains several cvs administration files.

As you go through the procedure, make sure that any files and directories you create have the correct group id and access controls for your purposes.

Note: To make these instructions easier to follow, several of the messages sent by cvs are eliminated in this example.

Let us assume you have a project named ours. It uses one source repository named foo, which is in a root directory called/codes/our sources. The project description file is /codes/our_sources/OurPD. The repository contains two subdirectories named a_dir and b_dir:

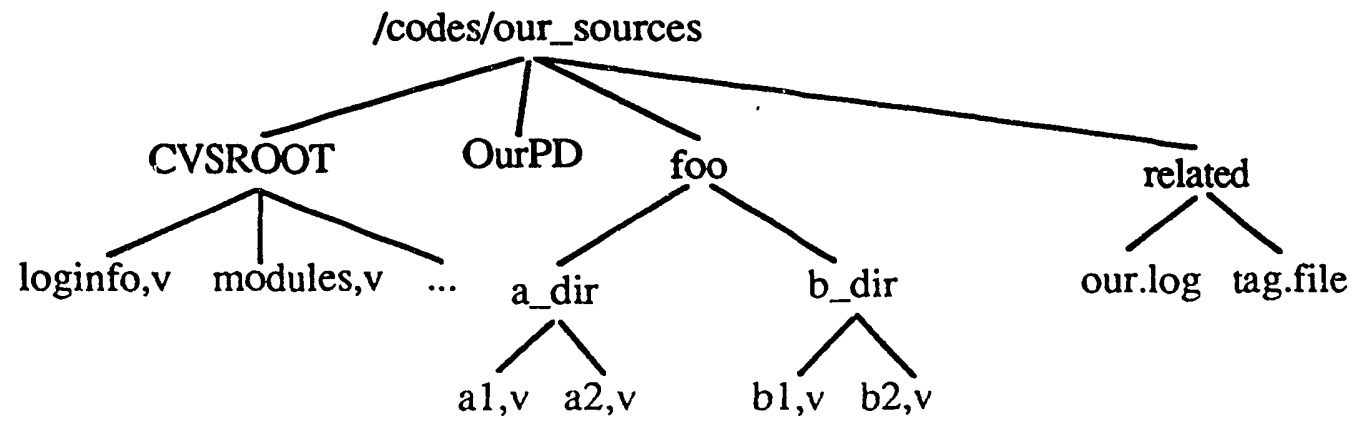

The project description file for this example looks like this:

\# Project description file for the OURS project.

\#

project ours

group our_group

access group

mail access

lock /codes/our_sources/related

pack all

print -Pmy.printer

tag /codes/our_sources/related/tag.file

root_dir/codes/our_sources

repository foo 


$$
\begin{aligned}
& \text { repository end } \\
& \text { root_dir end }
\end{aligned}
$$

You wish to add a root directory named/stuff/other_sources to the project. It does not yet exist. It is to contain two repositories named boo and bar.

First, set the environment variable CVSROOT to the name of your new root directory. This needs to be set before running cvs.

$$
\text { setenv CVSROOT /stuff/other_sources }
$$

Run cvsinit to create the root directory and set up the administration files.

cvsinit

Now we need to edit the administrative files, such as modules and loginfo. These were created by cvsinit. Also, samples of these files come with cvs. If you know where the cvs source directory is on your machine, look in a subdirectory called examples.

Move to some directory not contained in /stuff/other_sources. Let us assume it is called /home/me/work. Check out CVSROOT.

$$
\begin{aligned}
& \text { cd /home/me/work } \\
& \text { cVs checkout CVSROOT } \\
& \text { cd CVSROOT }
\end{aligned}
$$

The directory structure should now look like this:

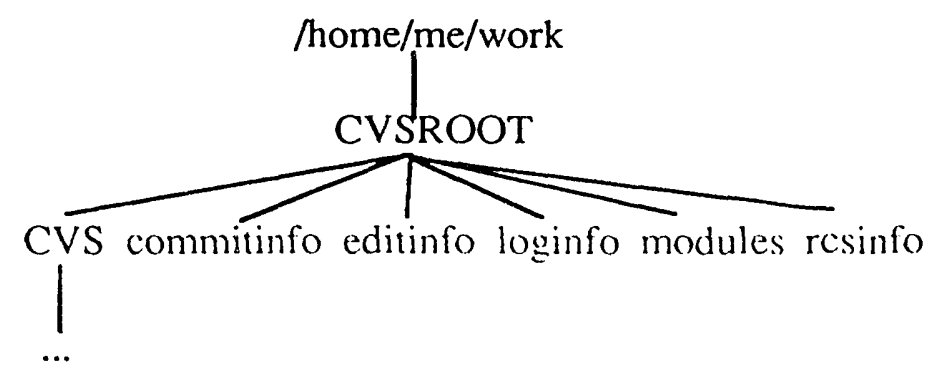

Edit any of the: files as needed.

The loginfo file can use any sort of filter program, such as cat, to send output to a log file. Here is an example that uses a local script called cmlog: 


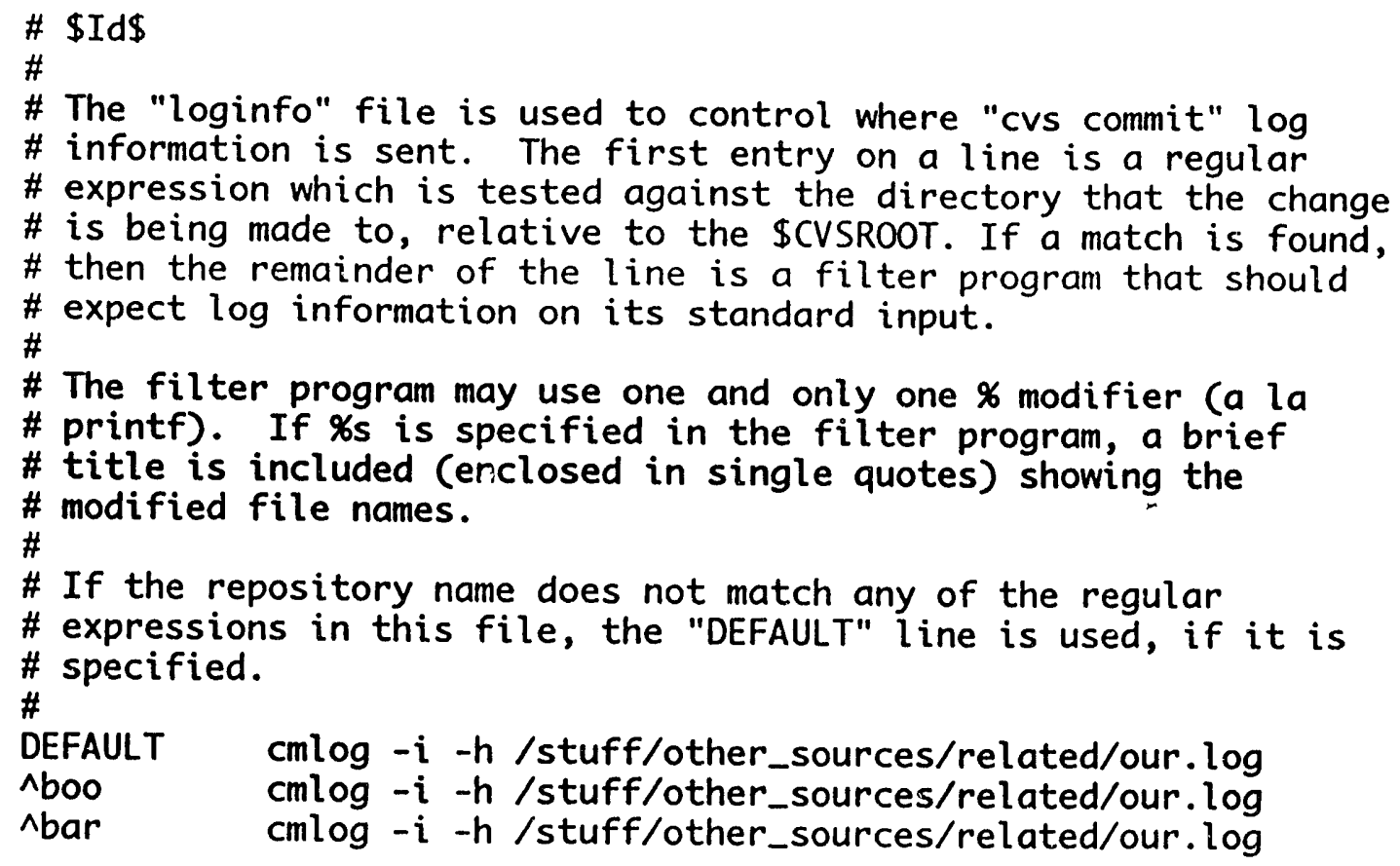

The modules file should loo'k something like this: 


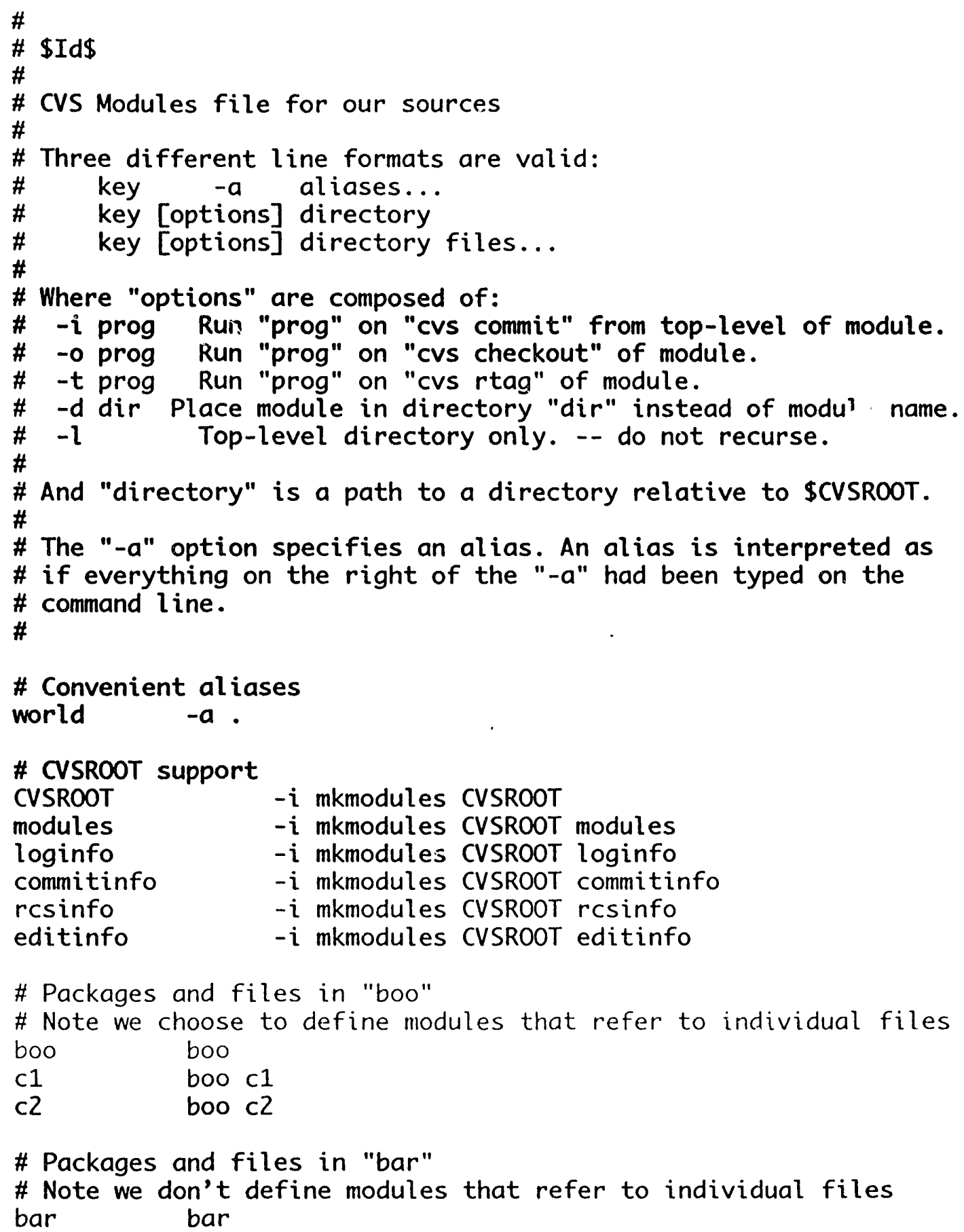

For more information on how to set up a modules file for CAVEMAN, see "Appendix C: The Modules File".

You may want to create a crsignore file, which is used to specify a list of files to ignore during update. I recommend creating this file, even if it only contains a blank line. If you don't, cvs tells you at almost every opportunity how nice it would be to have a cvsignore file. 
Create the cvsignore file in the /home/me/work/CVSROOT directory. Then type the following:

$$
\text { cl's add -m "New cvsignore file." cvsignore }
$$

Now, commit all the changes:

$$
\text { cVs commit -m "Initial changes to CVSROOT files." }
$$

You may now remove the CVSROOT directory in /home/me/work.

The source needs to be prepared. Put the source in some directory not contained in our_sources. Let us assume it is /home/me/work. Divide the source into directories and files, with the same hierarchy as you want them maintained by crs.

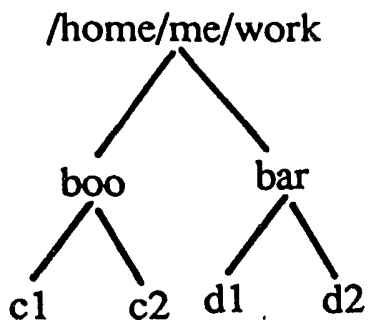

Optional: Put a comment line containing "\$Id\$" at or near the top of each file. cvs then places information about the version number and date into the comment line whenever the file is checked in. Suppose you insert a line that looks like this in file c1:

$$
\text { c } \$ I d \$
$$

After the file is placed under cave control, the line looks something like this:

$$
\text { c \$Id: c1,v } 1.0 \text { 1992/02/02 13:53:36 me Exp } \$
$$

Optional: Put a comment line containing "\$Log\$" at or near the top of each file. crs puts a log message at this location each time the file is committed. The log messages are cumulative.

The process of adding the $\$ \mathrm{Id} \$$ and $\$ \mathrm{~L}$ og $\$$ can be automated by using cave header.

Move to /home/me/work/boo, the top level directory of your first source. Assume the value of the version number in /codes/our_sources/related/tag.file is " $v$ _1_22". To install the source, with a tag of "BOO" and a version number of " $v_{-} 1 \_22$ ", type:

$$
\text { cvs import -m 'Initial installation of boo.' boo BOO v_1_22 }
$$

Move to /home/me/work/bar, and do the same: 
cvs import $-m$ 'Initial installation of bar.' bar BAR v_1_22

The directory/stuff/other sources/boo should now contain files that have the same names as the source files, with a ", $v$ " appended. The root directory should look like:

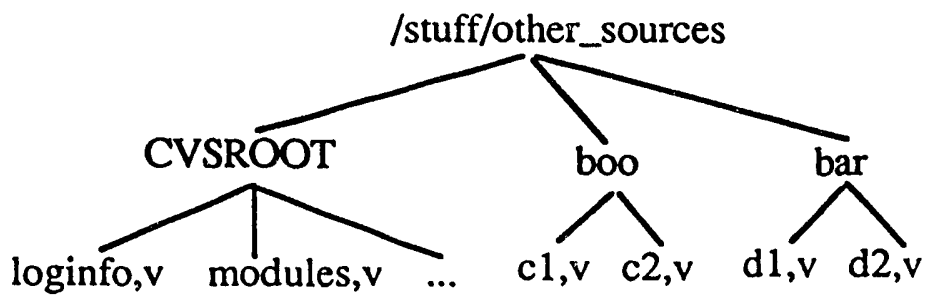

Make sure that any files and directories you created have the correct group id and access controls for your purposes. You may want to use the shell command:

\section{chmod $-R$ g+s /stuff/other_sources}

This forces any committed files to be the correct group. This ensures that any files committed in the future will have the correct group in the repository.

You may now remove the files in /home/me/work.

Add the new root directory information to the project description file, /codes/our_sources/OurPD:

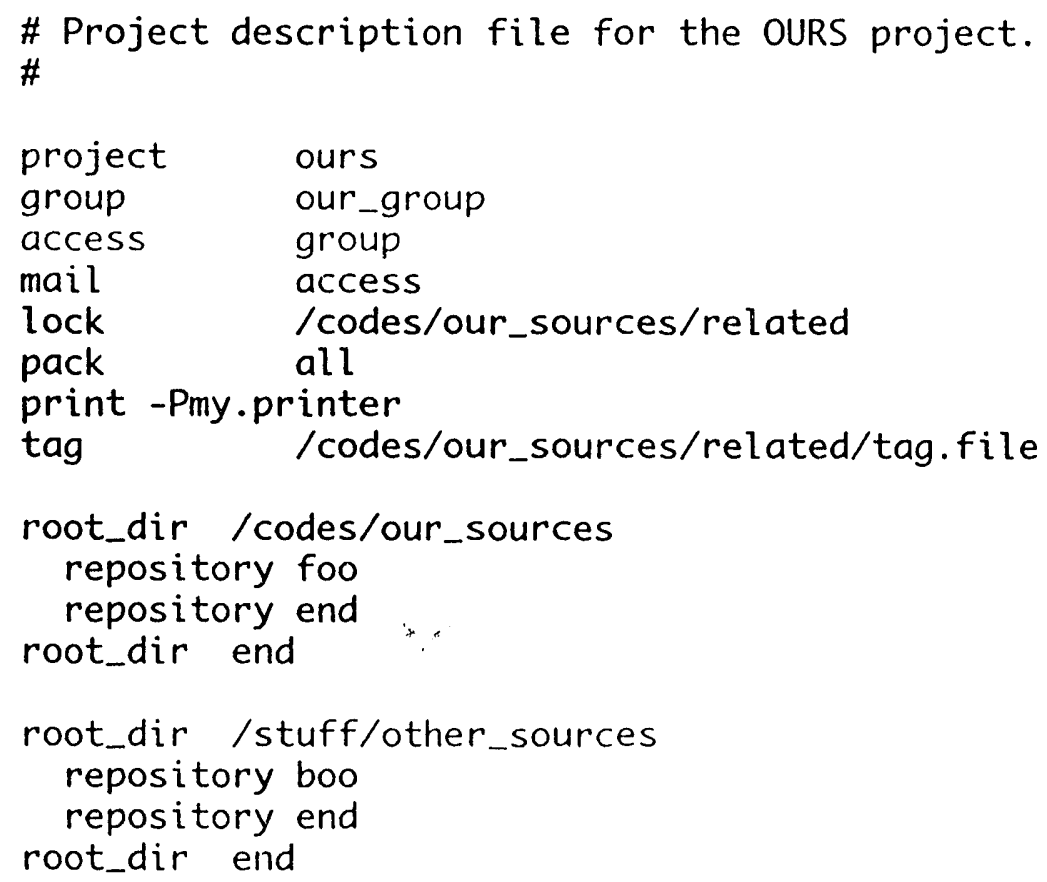




\section{Adding a Repository to an Existing Root Directory}

A root directory is a directory that contains cvs repositories and a subdirectory named CVSROOT which contains several cvs administration files.

Let us assume you have a root airectory called /codes/our_sources and it contains one repository named foo, the cvs administrative directory called CVSROOT, and a directory containing related files. The project description file used by cave is called OurPD.

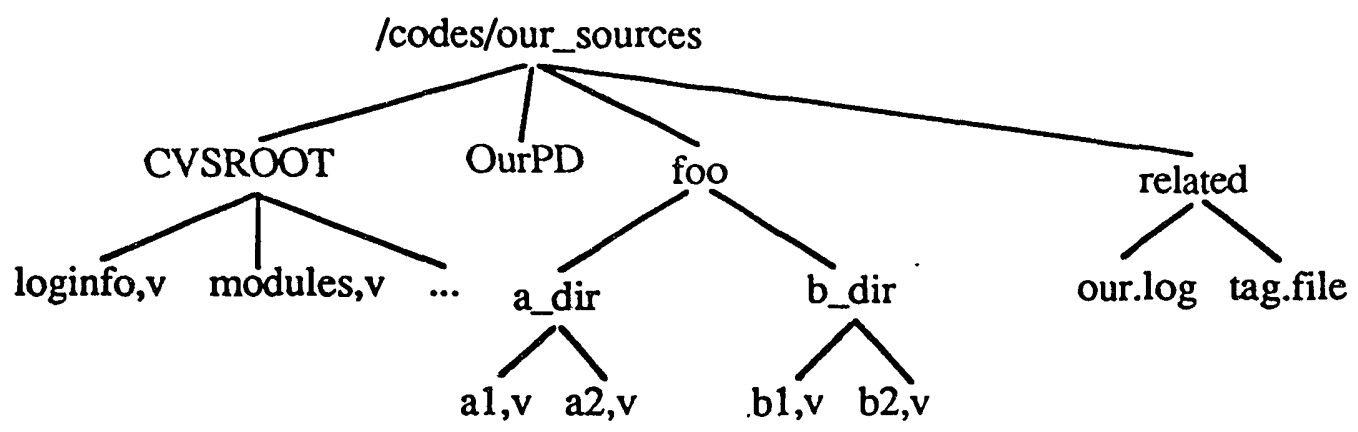

You wish to add a second repository named fum. The repository is to contain two files namedb1 and b2.

As you go through the procedure, make sure that any files and directories you create have the correct group id and access controlsfor your purposes.

Note: To make these instructions easier to follow, several of the messages sent by crs are eliminated in this example.

First, set the environment variable CVSROOT to the name of your root directory. This needs to be set before running cvs. You may want to set this in your .cshrc file.

setenv CVSROOT /codes/our_sources

Let us create the new repository.

$$
\begin{aligned}
& \text { cd /codes/our_sources } \\
& \text { mkdir fum }
\end{aligned}
$$

The source needs to be prepared. Put the source in some directory not contained in our_sources, such as /home/me/work. Divide the source into directories and files, with the same hierarchy as you want them maintained by cvs. 


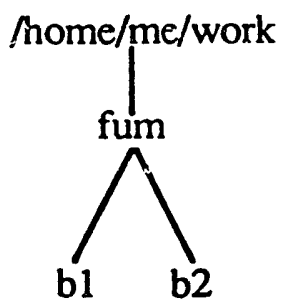

Optional: Put a comment line containing "\$Id\$" at or near the top of each file. cvs then places information about the version number and date into the comment line whenever the file is checked in. Suppose you insert a line that looks like this in file b1:

c \$Id\$

After the file is placed under cave control, the line looks something like this:

c SId: b1,v 1.0 1992/C2/04 11:53:46 me Exp \$

Optional: Put a comment line containing "\$LOg $\$$ " at or near the top of each file. cvs puts a log message at this location each time the file is committed. The lcg messages are cumulative.

The process of adding tl e \$Id\$ and \$Log $\$$ can be automated by using cave header.

Move to /home/me/work/fum, the top level directory of your source. To install the source, with a tag of "FUM" and a version number of " $v_{-} 1$ _o", type:

cvs import -m 'Initial installation of fum.' fum FUM v_1_0

The directory/codes/our sources/fum should now contain files that have the same names as the source files, with a ",v" appended. The root directory should look like:

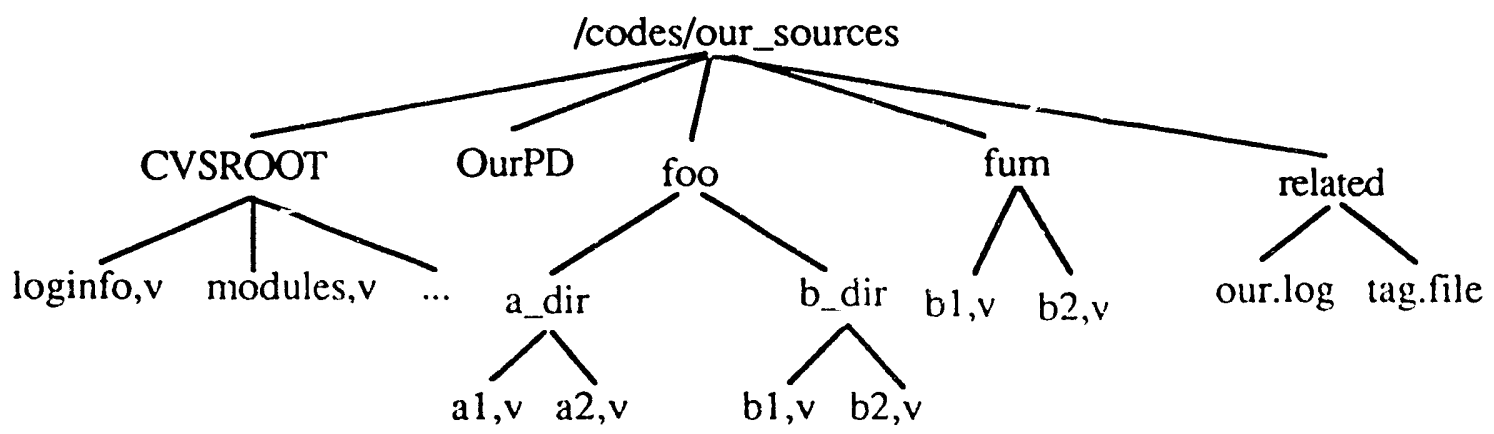


You may now remove the directory /home/me/work/fum.

Now we need to edit the administrative files, such as modules and loginfo. Move to some directory not contained in /codes/our sources. Let us assume it is called

/home/me/work. Check out CVSROŌT.

cd /home/me/work

cVs checkout CVSROOT

cd CVSROOT

The directory structure should now look like this:

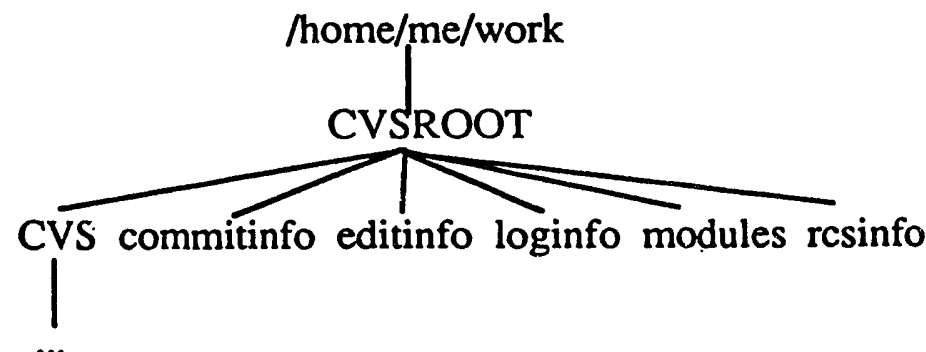

Edit any of these files which need to know about the new repository.

Now, commit the changes:

cVs commit $-m$ "Changes to CVSROOT files for new repo fum."

You may now remove the CVSROOT directory in /home/me/work.

Don't forget to edit the project description file/codes/our_sources/OurPD. It should look something like this:

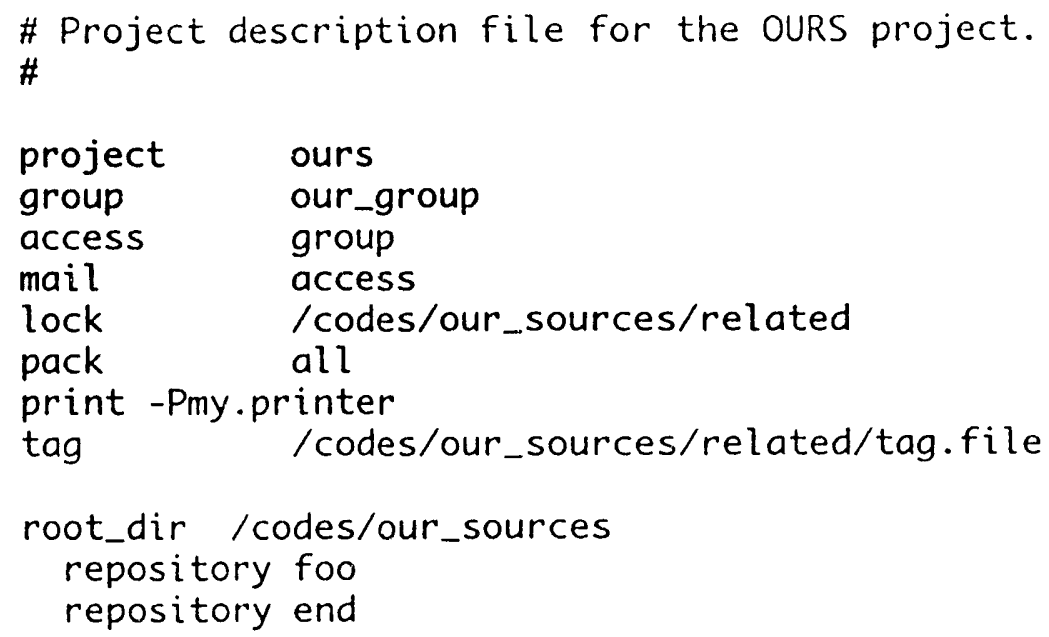




$$
\begin{aligned}
& \text { repository fum } \\
& \text { repository end } \\
& \text { root_dir end }
\end{aligned}
$$

Make sure that any files and directories you created have the correct group id and access controls for your purposes. 


\section{Retrieving Files From a Repository}

Use the cave checkout command to retrieve files from a repository.

The checked out source files are placed in a directory named after the project, as determined from the project description file. They are placed so that the structure echoes that of the root directory.

Let us assume you have a project named ours. It uses one source repository named foo, which is in a root directory called /codes/our sources. The project description file is /codes/our_sources/OurPD. The repository contains two subdirectories named a_dir and b_dir:

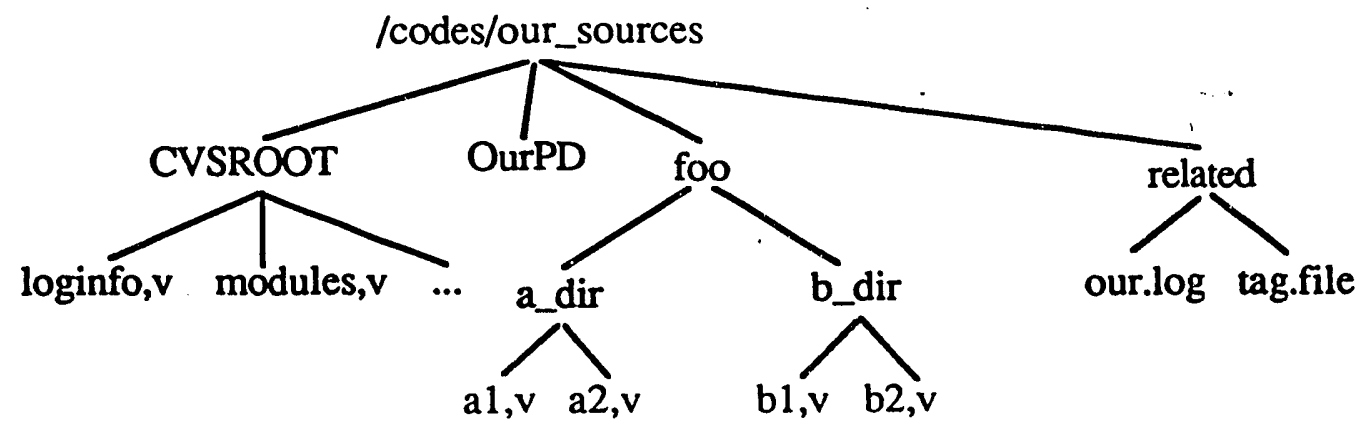

The project description file for this example looks like this:

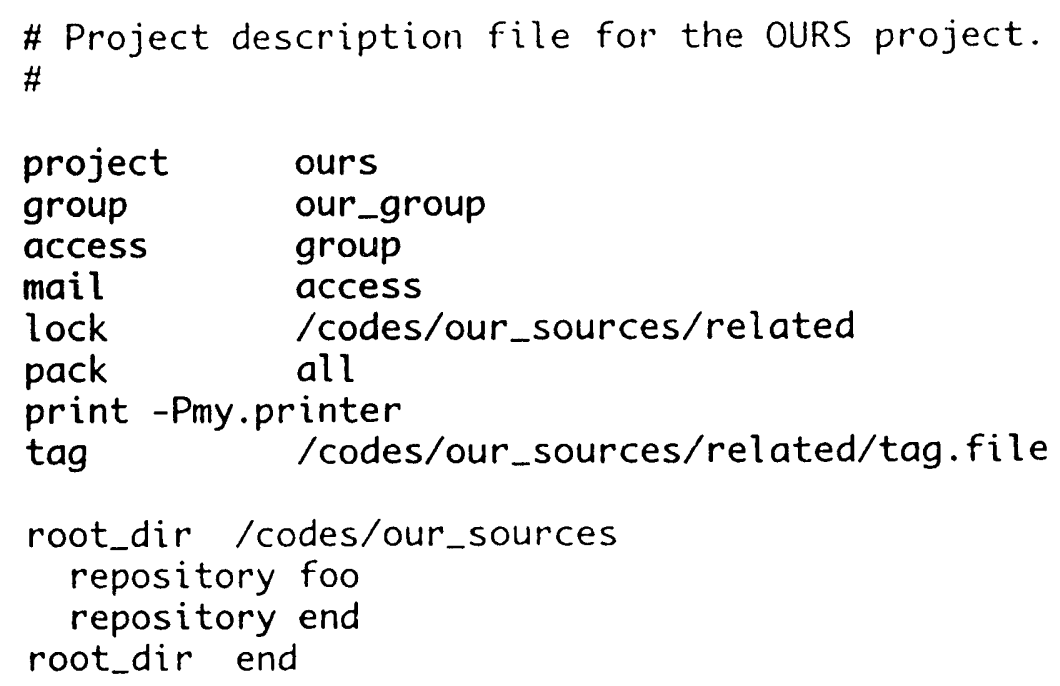


While in a directory named /home/me/work, which is empty, you decide to check out the subdirectory a_dir. You can type:

cave - $d$ /codes/our_sources/OurPD checkout foo/a_dir

Assume you had earlier set the environment variable CAVE_PD to the path name of the project description file:

setenv CAVE_PD /codes/our_sources/OurPD

Then the call to cave checkout could have been shorter:

cave checkout foo/a_dir

If the modules file contained a key for a_dir, such as:

a_dir foo/a_dir

You could have typed:

cave checkout a_dir

And if you like to type as little as possible, you could have used one of the synonyms for checkout, such as co or get.

cave co a_dir

However you type the command line, the following directory structure results:

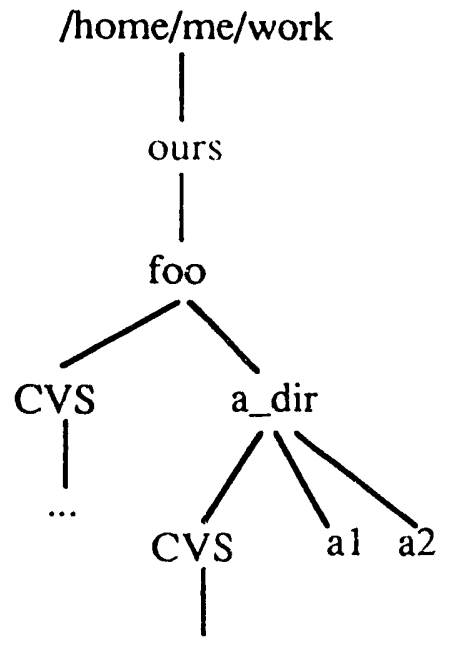


Note also that the project name ours is used to name a directory which contains a structure resembling that of the root directory. Many of the commands in cave look for a directory named after the project in order to orient themselves within the local directory structure.

You may now make changes to the a_dir directory, such as editing files, adding files and removing files. Do not make any changes to the CVS directory.

Since you don't want to type the name of the project description file every time you run cave, let us assume that you set the environment variable CAVE_PD, as described above.

Now, you decide to check out the b_dir subdirectory as well. While in /home/me/work, or anywhere in the directory chain headed by ours, run cave again:

cave checkout our_sources/foo/b_dir

The directory structure now looks like:

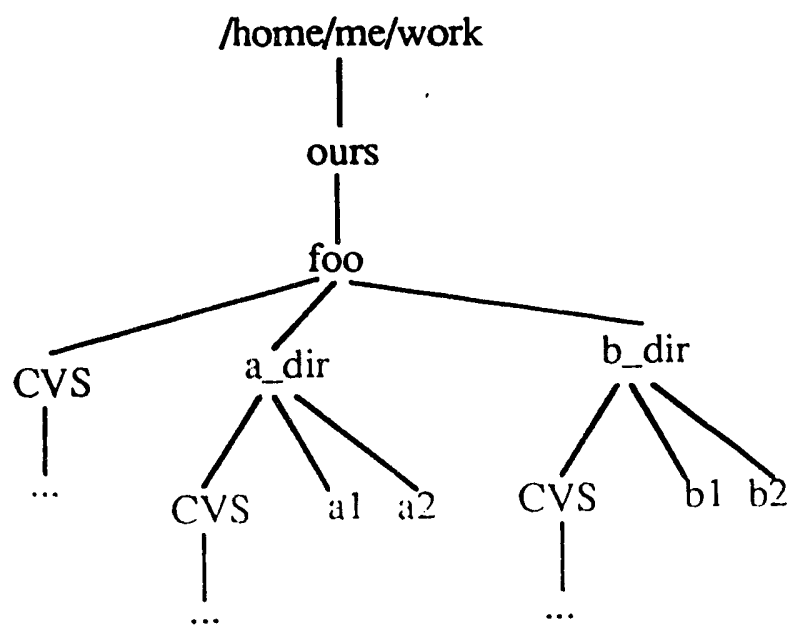

Now, let us look at the case where a project has more than one root directory.

Suppose you have the same root directory described above-/codes/our_sources. You also need to use a root directory named/stuff/other_sources. The project description file is still /codes/our_sources/OurPD, but now it lists Tcodes/our_sources as the first root directory, and /stuff/other_sources as the second root directory. The second root directory looks like: 


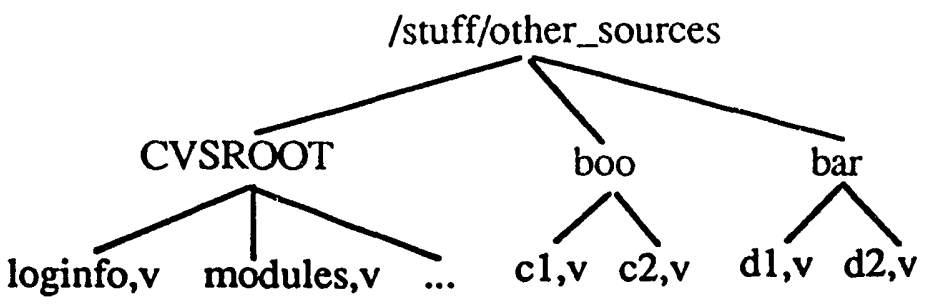

The project description file for this example looks like:

\# Project description file for the OURS project.

$\#$

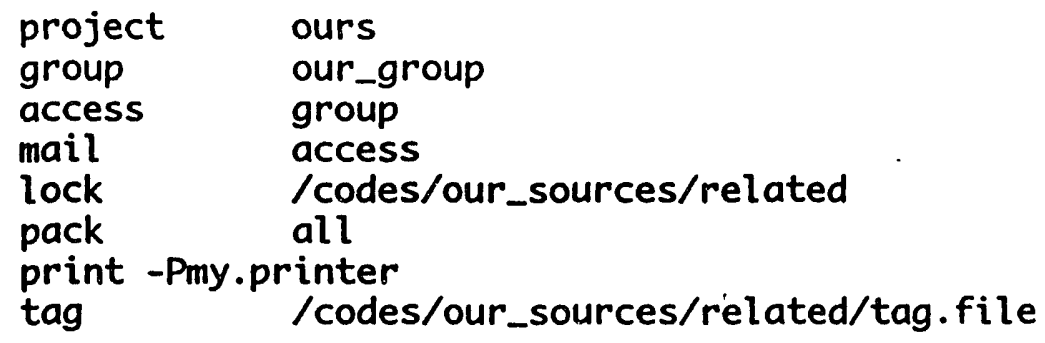

While in a directory named /home/me/work, which is empty, you decide to check out the subdirectory a_dir from the root directory /codes/our_sources:

cave checkout our_sources/foo/a_dir

The following directory structure results: 


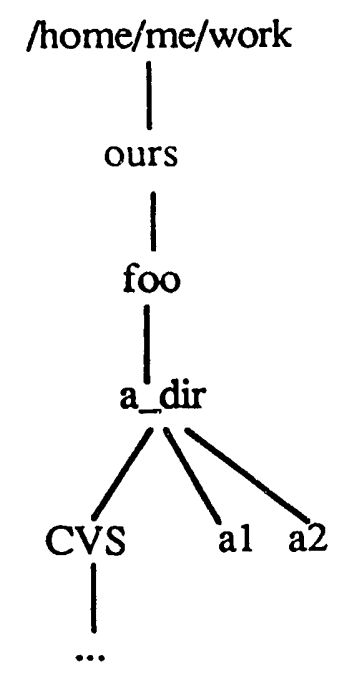

Now you decide to check out boo from the second root directory:

cave checkout other_sources/boo

The following directory structure results:

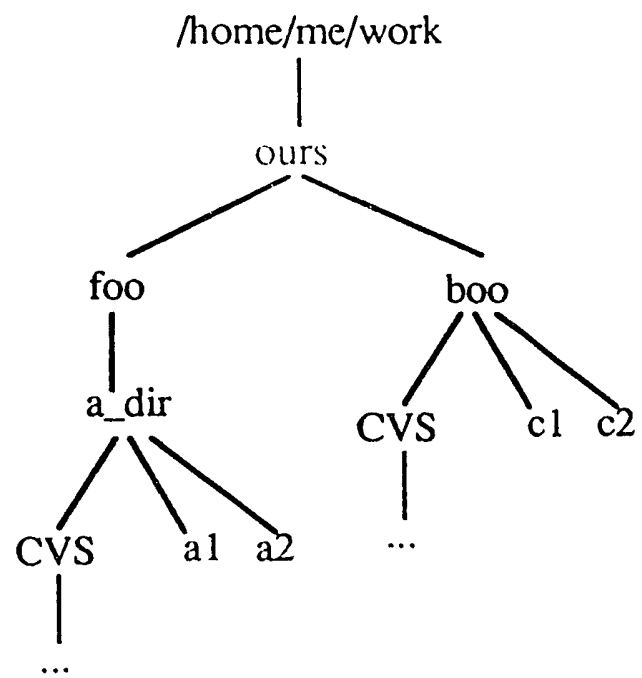


Now you decide to check out file b1 from the subdirectory b_dir in the root directory our_sources:

cave checkout our_sources/foo/b_dir/b1

The following directory structure results:

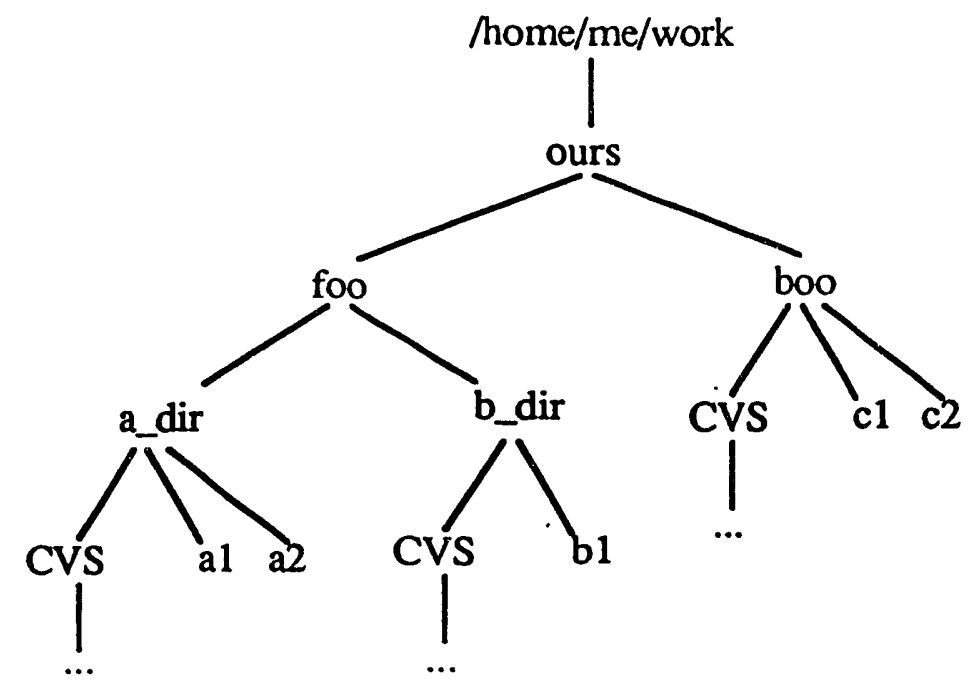

The project description file is described in the chapter "Project Description File". 


\section{Adding a Directory to a Repository}

Use the cave add command to add files and directories to a repository.

Let us assume you have a project named ours. It uses one source repository named foo, which is in a root directory called/codes/our_sources. The project description file is tcodes/our_sources/OurPD. The repository contains two subdirectories named a_dir and b_dir:

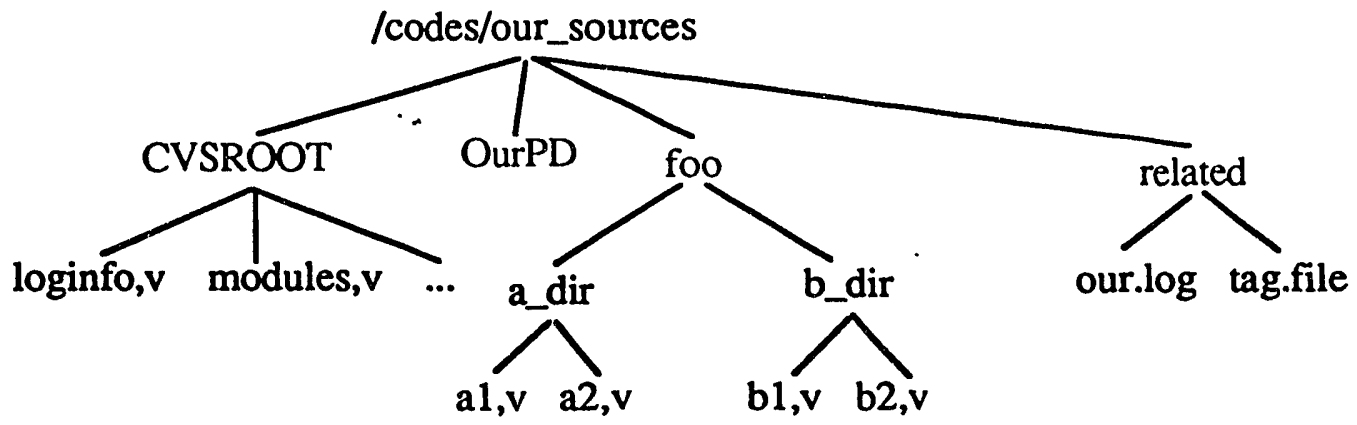

You wish to add another directory to foo named c_dir that contains the files $\mathbf{c l}$ and $\mathbf{c 2}$.

As you go through the procedure, make sure that any files and directories you create have the correct group id and access controls for your purposes.

Note: To make these instructions easier to follow, several of the messages sent by cave and crs are eliminated in this example.

Since you don't want to type the name of the project description file every time you run cave, let us assume that you have set the environment variable CAVE_PD:

$$
\text { setenv CAVE_PD /codes/our_sources/OurPD }
$$

Move to your working directory. Let us assume it is called/home/me/work. Check out something from foo, such as a_dir, so that the CVS directory is created. Do not change anything in any directory named CVS.

$$
\text { cave checkout foo/a_dir }
$$

If the modules file contained a key for a dir, such as:

$$
\text { a_dir foo/a_dir }
$$


And if you used one of the synonyms for checkout, such as co or get, you could have typed:

cave co a_dir

However you type the command line, the following directory structure results:

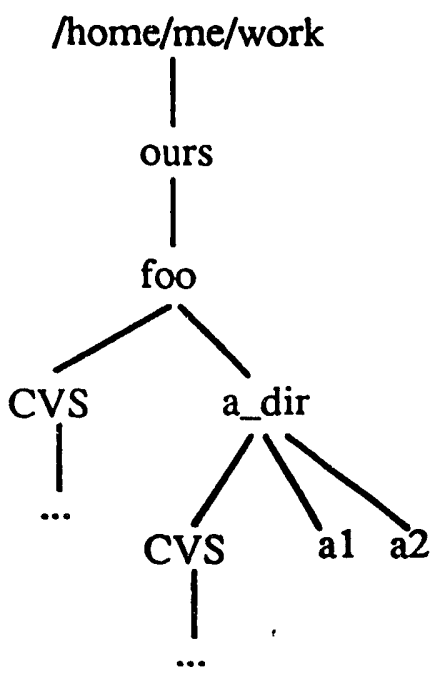

The source needs to be prepared. Move to /home/me/work/ours/foo. Create a new directory:

$$
\begin{aligned}
& \text { mkdir c_dir } \\
& \text { cd c_dir }
\end{aligned}
$$

Divide the source into files, with the same hierarchy as you want them maintained by cvs. 


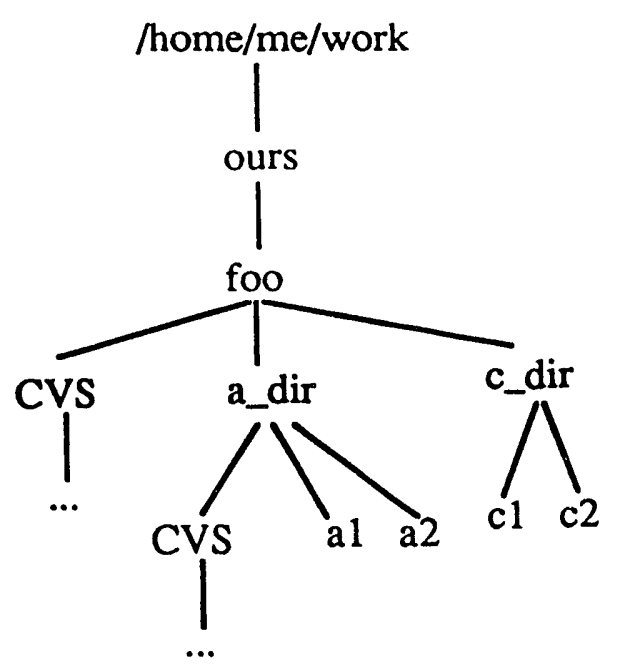

Optional: Put a comment line containing "\$Id\$" at or near the top of each file. cvs then places information about the version number and date into the comment line whenever the file is checked in. Suppose you insert a line that looks like this in file c1:

\section{c $\$ I d \$$}

After the file is placed under cave control, the line looks something like this:

c \$Id: c1,v $1.01992 / 02 / 06$ 14:03:16 me Exp \$

Optional: Put a comment line containing " $\$ \log \$$ " at or near the top of each file. cvs puts a log message at this location each time the file is committed. The log messages are cumulative.

The process of adding the $\$ I d \$$ and $\$ L \log \$$ can be automated by using cave header.

Move back up a level to foo:

$$
\text { cd .. }
$$

Run cave add. Note that running cave add does not place the files in the repository. It simply marks these files as new files that will be committed in the future.

$$
\text { cave add -R c_dir }
$$

Now the local directory should look like this: 


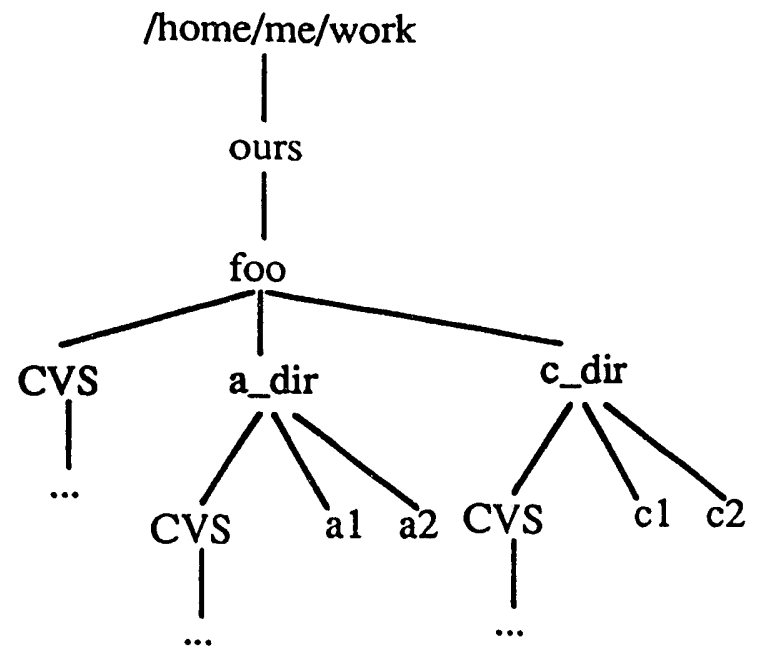

The repository /codes/our_sources/foo should now contain c_dir, but not its files:

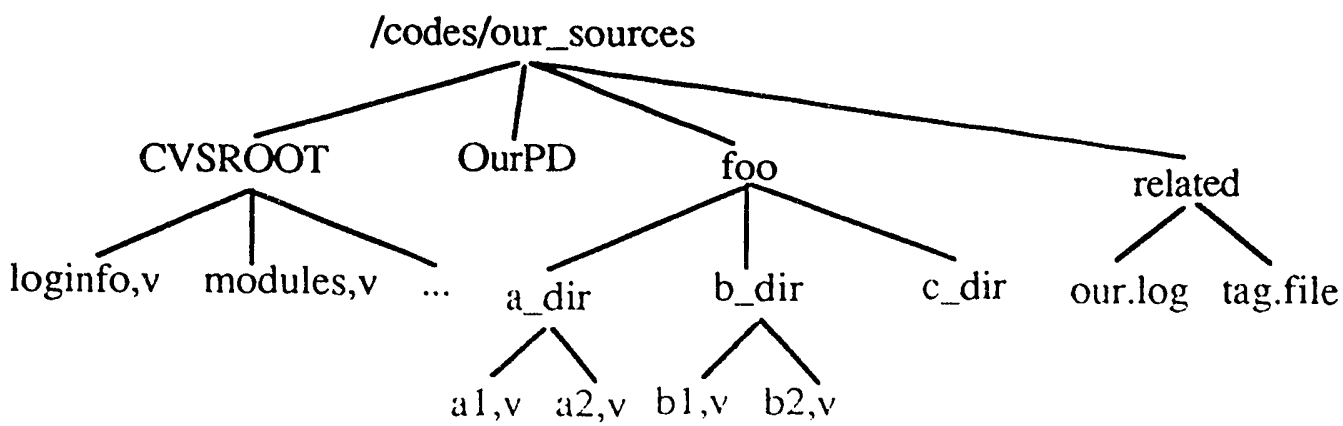

Remember, you may want to update the modules file from CVSROOT so that it knows about the new directory. If so, check out modules:

$$
\text { cave co modules }
$$

Now the local directory should look like this: 


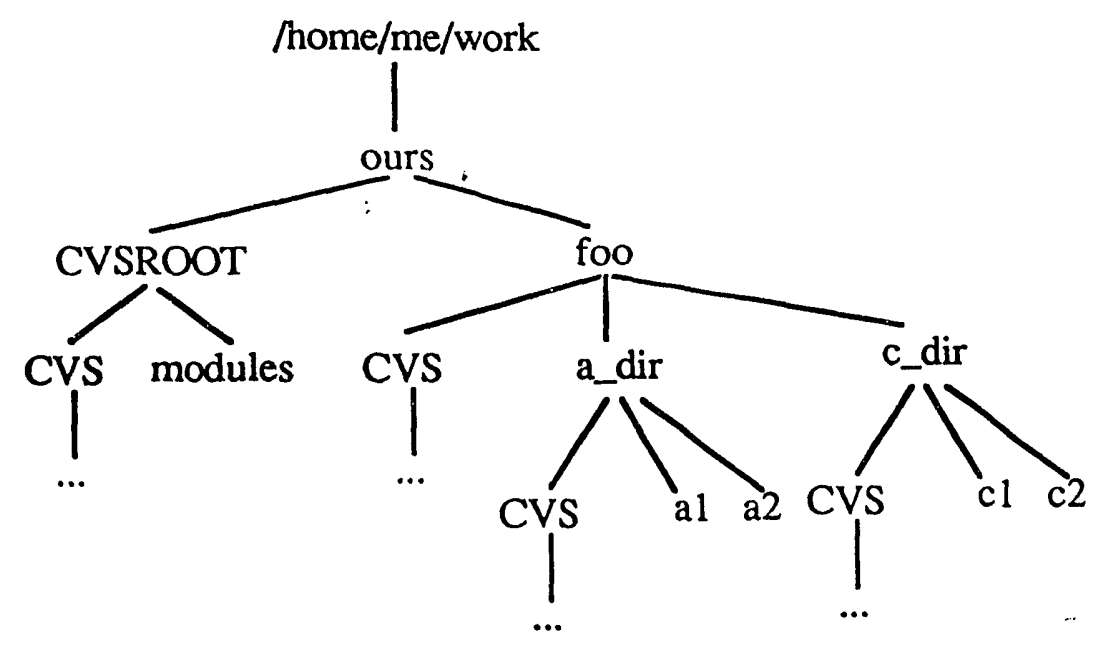

Make any necessary changes to the modules file.

When you are ready to make all the changes permanent, run cave commit from within /home/me/work/ours or one of its subdirectories:

cave commit -m 'Committing c_dir files' foo CVSROOT

The directory/codes/our_sources/foo should now contain c_dir and its files:

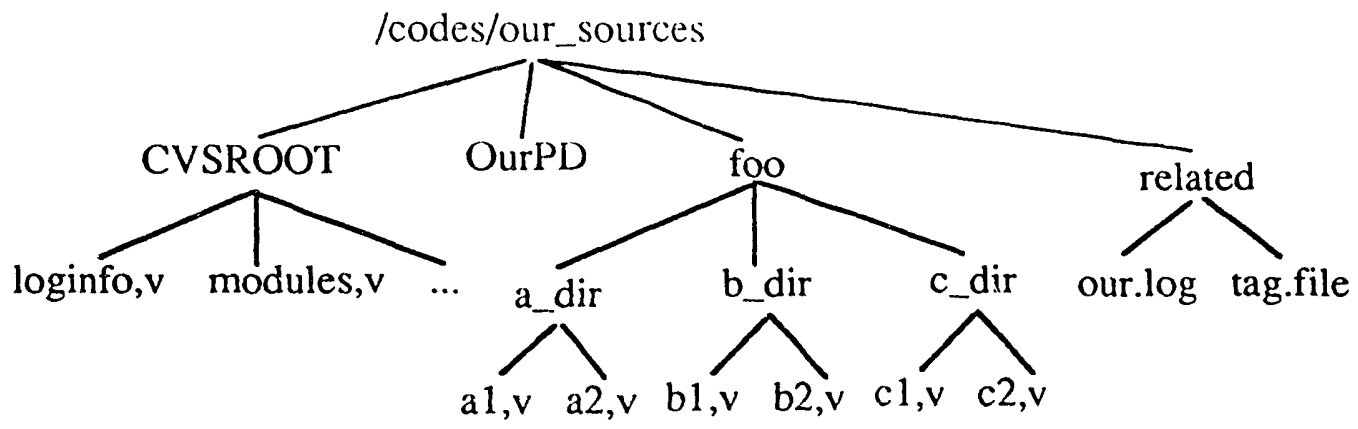

Make sure that any files and directories you created have the correct group id and access controls for your purposes.

You may now delete the directories in /home/me/work/ours. 


\section{Deleting a Directory From a Repository}

Directories cannot be deleted via cvs. The files within them may be removed, but the Attic directory must remain so that older versions of the files may be checked out.

If you decide that the directory is truly obsolete, and will never be needed, simply delete it the way you would any other UNIX directory. 


\section{Adding a File to a Repository}

Use the cave add command to add files and directories to a repository.

Let us assume you have a project named ours. It uses one source repository named foo, which is in a root directory called/codes/our_sources. The project description file is fcodes/our_sources/OurPD. The repository contains two subdirectories named a_dir and b_dir:

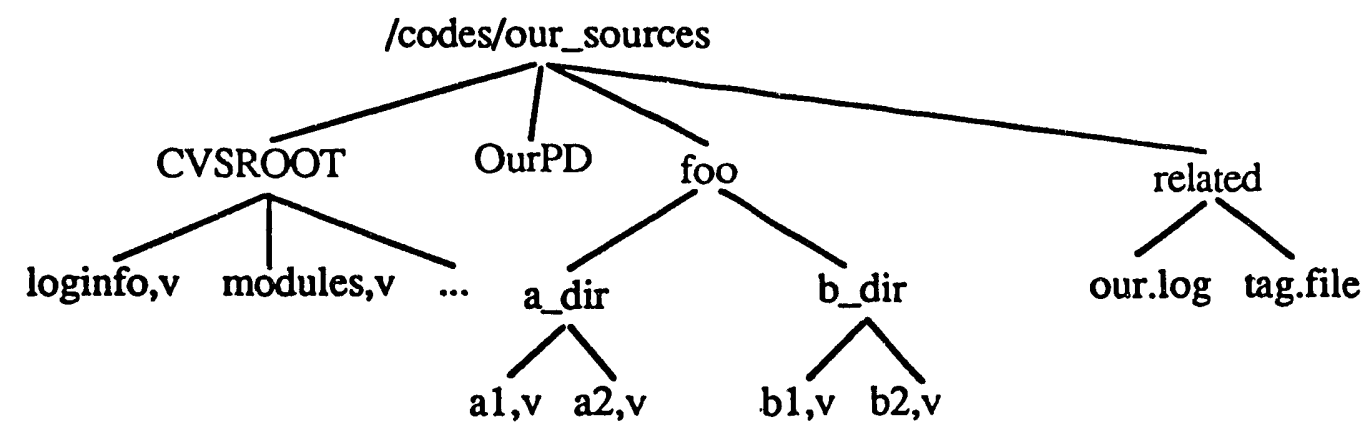

You wish to add another file to a_dir in foo named a3.

As you go through the procedure, make sure that any files and directories you create have the correct group id and access controls for your purposes.

Note: To make these instructions easier to follow, several of the messages sent by cave and crs are eliminated in this example.

Since you don't want to type the name of the project description file every time you run cave, let us assume that you have set the environment variable CAVE_PD:

$$
\text { setenv CAVE_PD /codes/our_sources/OurPD }
$$

Move to your working directory. Let us assume it is called /home/me/work. Check out a_dir, so that the CVS directory is created. Do not change anything in any directory named CVS.

cave checkout foo/a_dir

If the modules file contained a key for a dir, such as:

$$
\text { a_dir foo/a_dir }
$$


And if you used one of the synonyms for checkout, such as co or get, you could have typed:

cave co a_dir

However you type the command line, the following directory structure results:

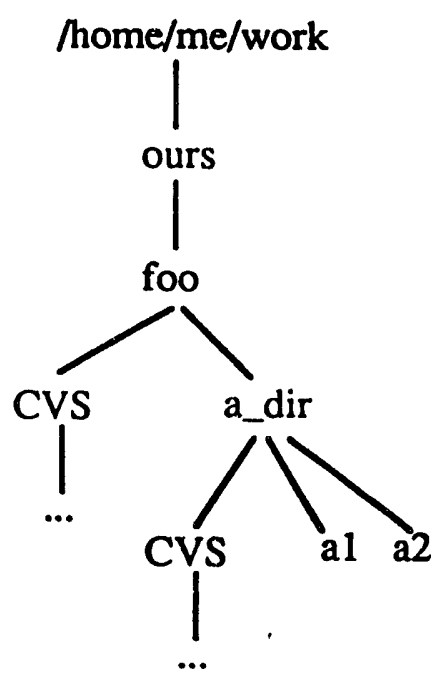

The source needs to be prepared. Move to /home/me/work/ours/foo/a_dir. Create the new file a3, or copy it in from some other location. 


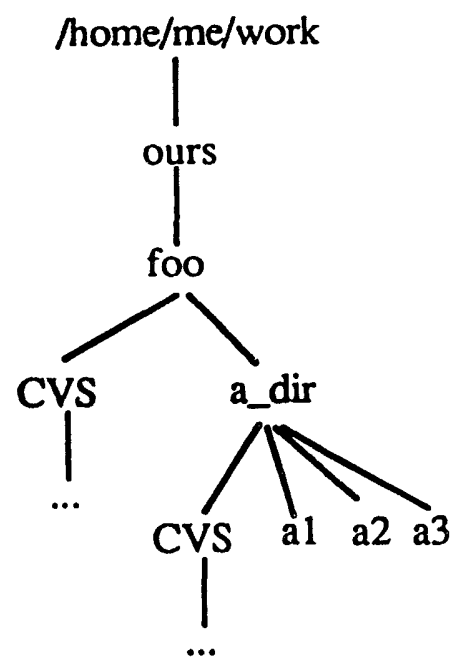

Optional: Put a comment line containing "\$Id\$" at or near the top of each file. cvs then places information about the version number and date into the comment line whenever the file is checked in. Suppose you insert a line that looks like this in file a3:

c \$Id\$

After the file is placed under cave control, the line looks something like this:

c \$Id: a3,v $1.01992 / 02 / 06$ 14:03:16 me Exp $\$$

Optional: Put a comment line containing "\$Log\$" at or near the top of each file. cvs puts a log message at this location each time the file is committed. The log messages are cumulative.

The process of adding the \$Id $\$$ and $\$ \log \$$ can be automated by using cave header.

Run cave add. Note that running cave add does not place the file in the repository. It simply marks the file as a new file that will be committed in the future.

$$
\text { cave add a3 }
$$

Remember, you may want to update the modules file from CVSROOT so that it knows about the new file. If so, check out modules:

$$
\text { cave co modules }
$$

Now the local directory should look like this: 


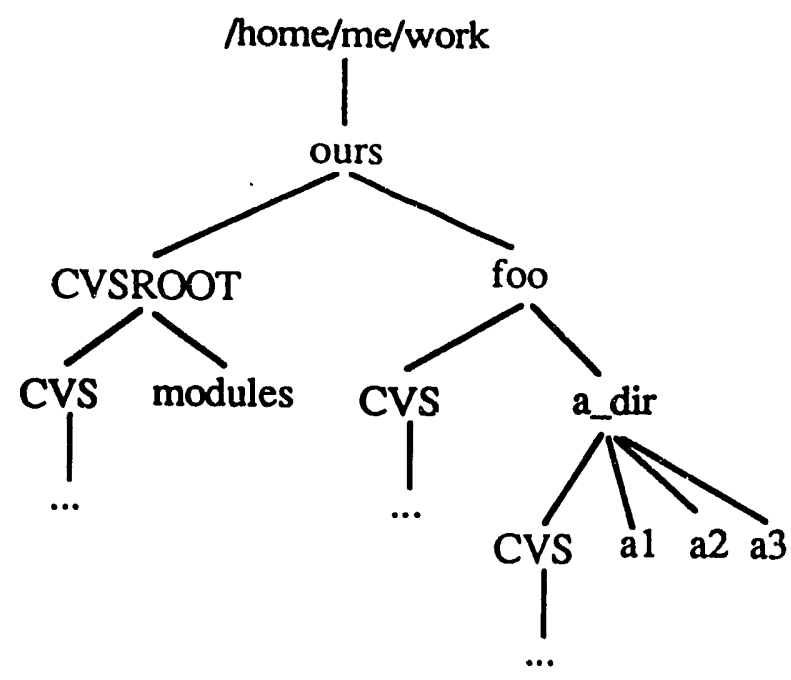

Make any necessary changes to the modules file

When you are ready to make all the changes permanent, run cave commit from within /home/me/work/ours or one of its subdirectories:

cave commit $-m$ 'Adding the file a_dir/a3' foo CVSROOT

The directory/codes/our_sources/foo should now contain the new file:

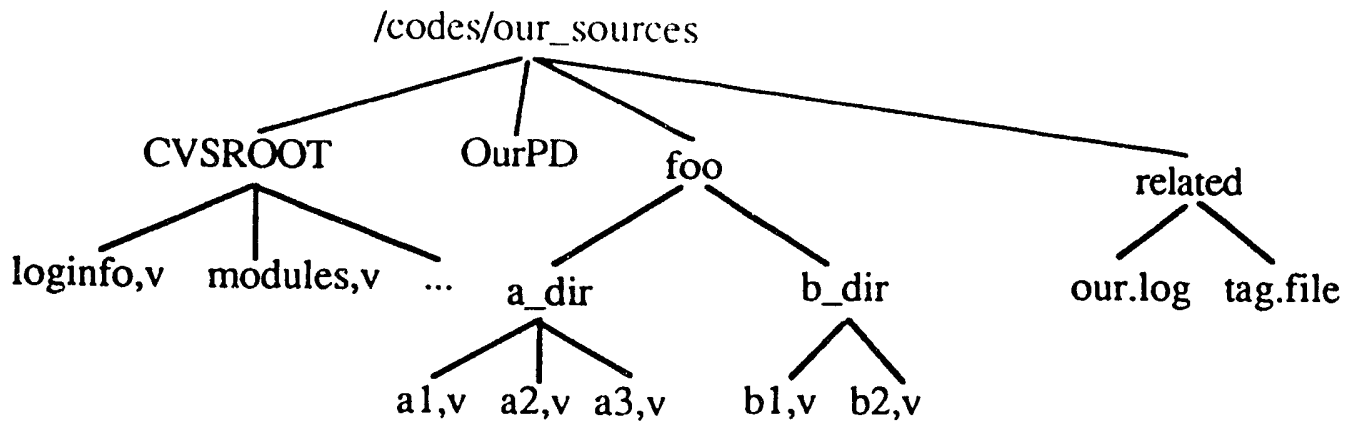

Make sure that any files and directories you created have the correct group id and access controls for your purposes.

You may now delete the directories in /home/me/work/ours. 


\section{Deleting a File From a Repository}

Use the cave remove command to delete files from a repository.

Let us assume you have a project named ours. It uses one source repository named foo, which is in a root directory called/codes/our sources. The project description file is /codes/our_sources/OurPD. The repository contains two subdirectories named a_dir and b_dir:

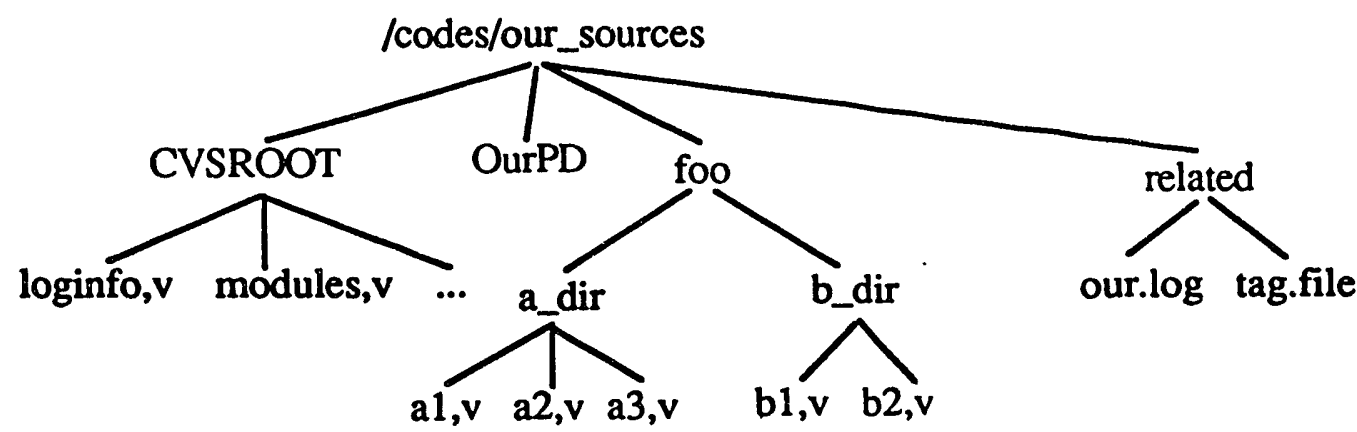

You wish to remove the file in foo/a_dir named a3.

Note: To make these instructions easier to follow, several of the messages sent by cave and cvs are eliminated in this example.

Since you don't want to type the name of the project description file every time you run cave, let us assume that you have set the environment variable CAVE_PD:

$$
\text { setenv CAVE_PD /codes/our_sources/OurPD }
$$

Move to your working directory. Let us assume it is called /home/me/work. Check out a_dir, so that the CVS directory is created. Do not change anything in any directory named CVS.

cave checkout foo/a_dir

If the modules file contained a key for a_dir, such as:

$$
\text { a_dir foo/a_dir }
$$

And if you used one of the synonyms for checkout, such as co or get, you could have typed: 
cave co a_dir

However you type the command line, the following directory structure results:

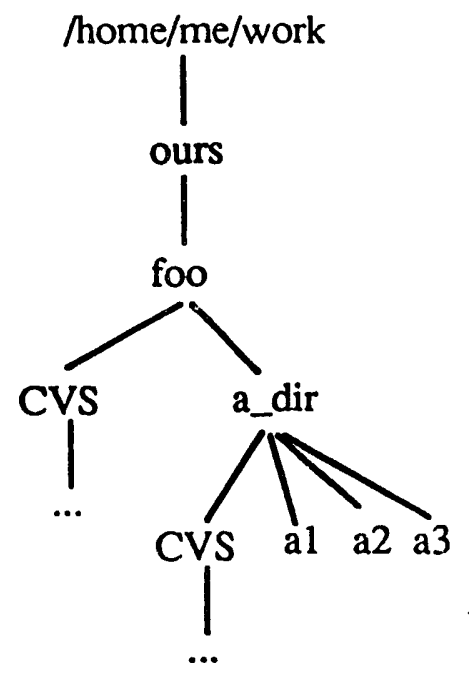

Move to /home/me/work/ours/foo/a_dir. Run cave remove. Note that running cave remove does not immediately remove the file from the repository. It simply marks the file as a file that will be removed in the future.

cave remove a3

Now the local directory should look like this: 


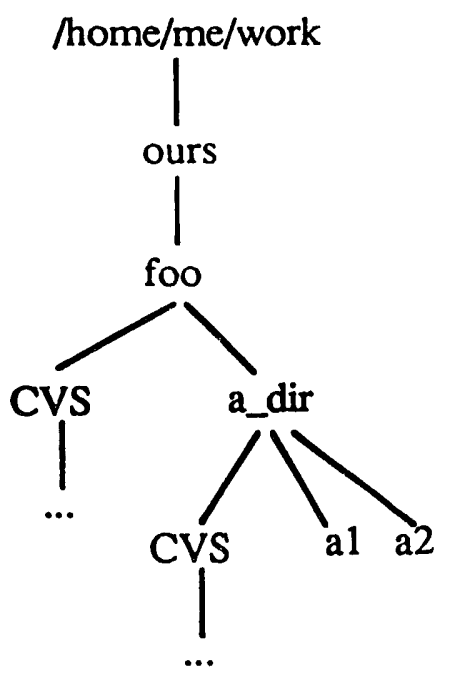

Remember, you may want to update the modules file from CVSROOT so that it knows about the new file. If so, check out modules:

$$
\text { cave co modules }
$$

Now the local directory should look like this:

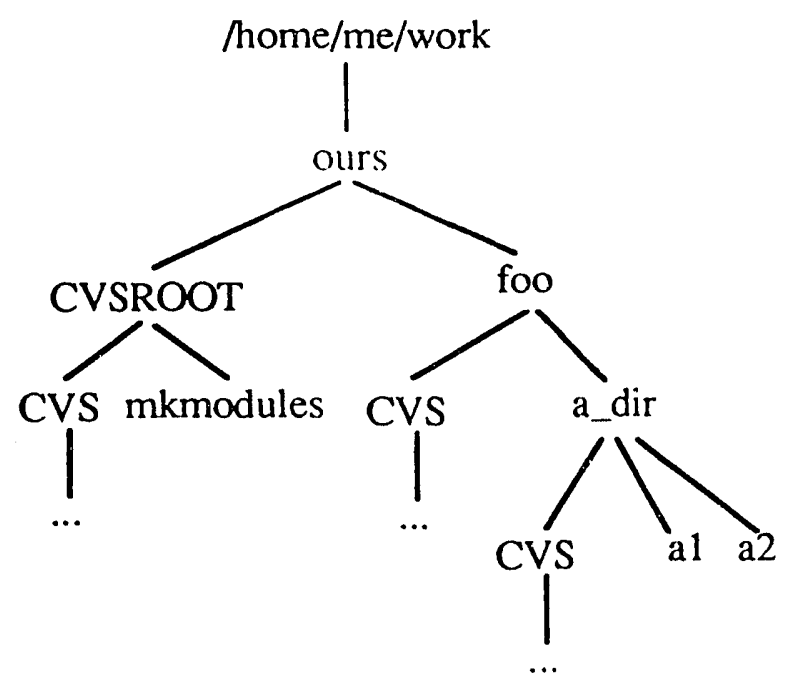


Make any necessary changes to the modules file

When you are ready to make all the changes permanent, run cave commit from within /home/me/work/ours or one of its subdirectories:

cave commit -m Removing the file a_dir/a3' foo CVSROOT

The directory /codes/our_sources/foo should now look like:

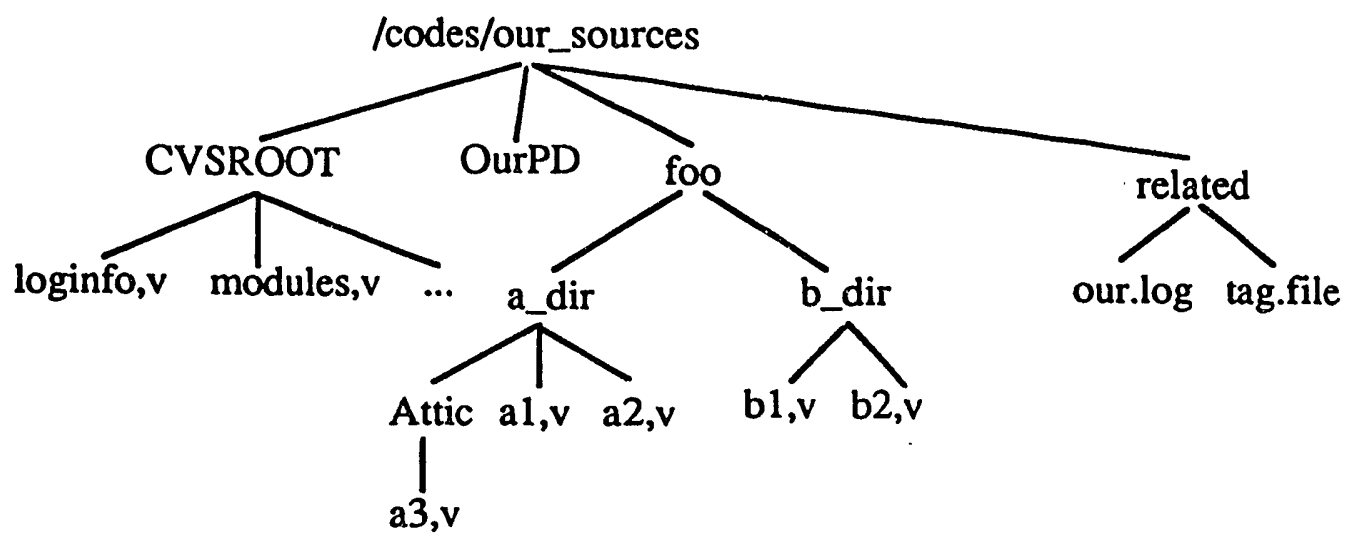

You may now delete the directories in /home/me/work/ours. 


\section{Appendix C: The Modules File}

The modules file is a special file used by cvs for administrative purposes. It is used to define symbolic names or aliases for files or groups of files. It exists in the directory \$CVSROOT/CVSROOT, where \$CVSROOT is the pathname to the root directory which hold the repositories.

A module is a name or key assigned in the modules file to reference subdirectories and files in a source repository. For example, you could define "my.pkg" to mean all the files in the directory "foo/my.pkg". The key may be different than the real name of the subdirectory or file in the source repository.

The modules file should look something like this:

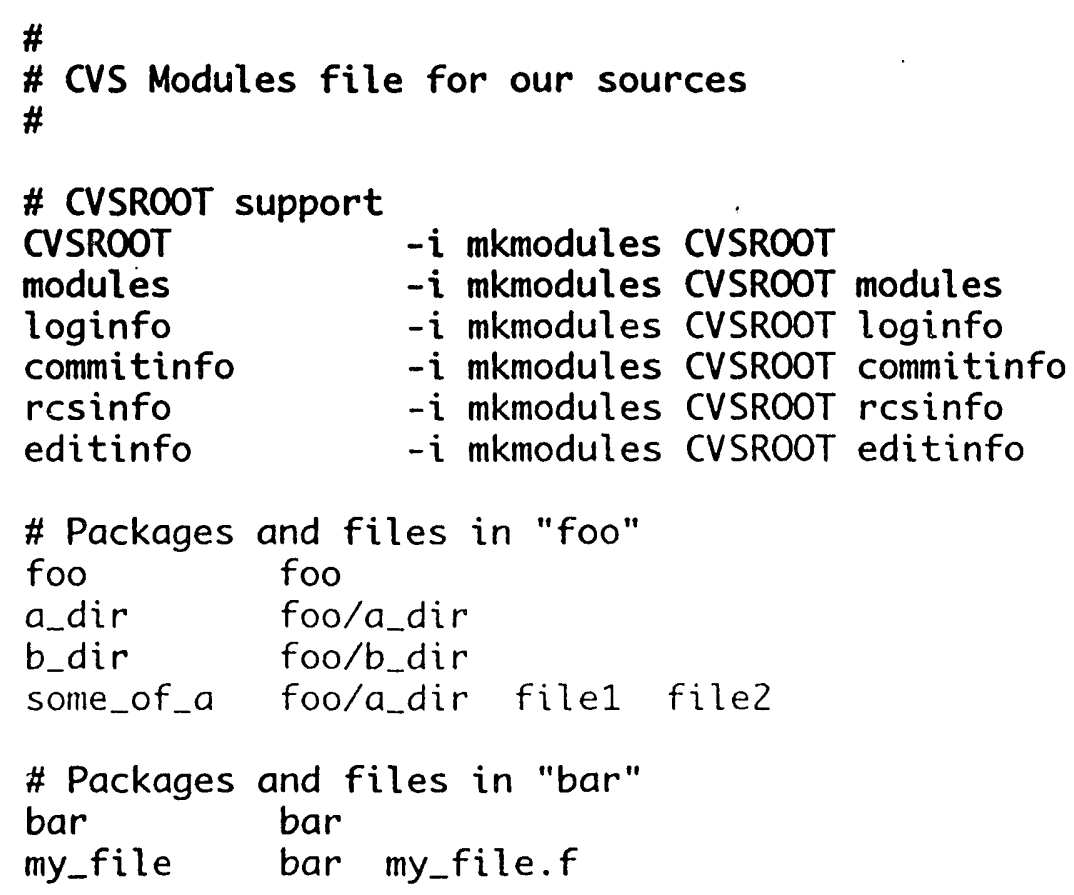

The six lines near the top containing the work "mkmodules" are standard for most modules files. They are there to make sure that the correct procedure is followed whenever any of the cvs administrative files are changed. Below these lines are the ones that define the modules. There is a repository named foo, and the key to it is unimaginatively named foo. To save some typing, keys have been defined for the directories foo/a_dir and foo/b_dir. So, to check out all of the files in foo/a_dir, we could type:

cave co foo/a_dir 
or, we could type:

cave co a_dir

There is also a module named some_of_a, which would get just filel and file2. So, we could type:

cave co foo/a_dir $\{$ file1,file2\}

or, we could type:

cave co some_of_a

cave forces any checked out files into a local directory structure which echoes that of the root directory. This is absolutely necessary, to ensure that later requests to pack or commit the files result in the proper actions. This behavior is different than cvs, which does not enforce any sort of local directory structure.

The way cave enforces the directory structure causes some of the fancier features of the modules file not to behave in the same manner as they do with plain cvs. Let us look at each of these line formats and options.

The standard modules file has the following header:

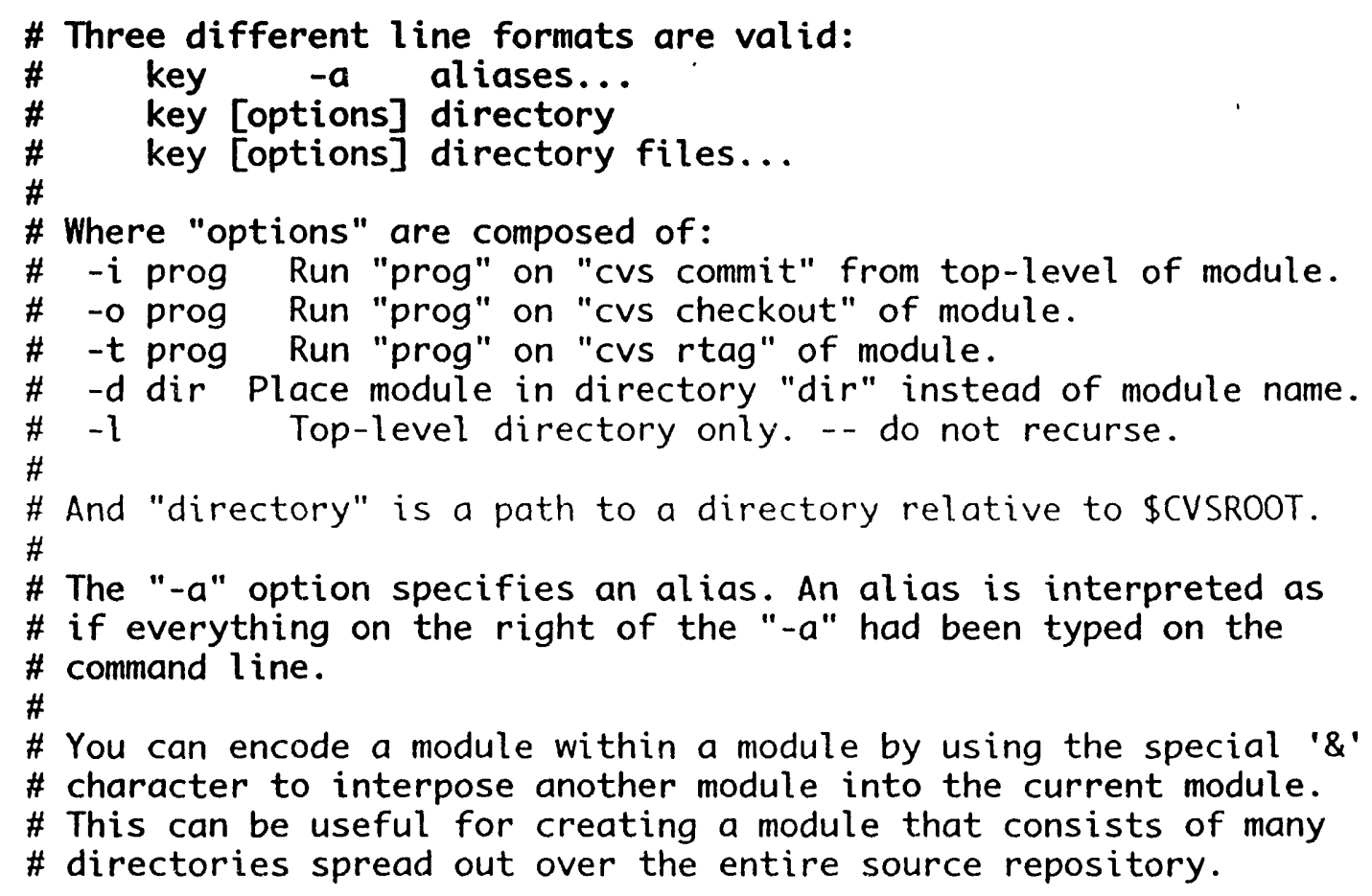

The simplest cases were shown in the earlier example:
key directory
$-\rightarrow$ b_dir
foo/b_dir
key directory files... $\quad .-\rightarrow$ some_of_a
foo/a_dir file1 filez 
The $-I$ and $-d$ options are regarded by cave as errors, as they would bypass the enforced directory structure.

The -i, -o and -t options only work if:

- The module key and the actual directory name are the same.

- The module refers to a directory which contains files in the next level down. There may be subdirectories present, but they will not be acted upon.

So if we had included the -i option in the earlier examples:

i

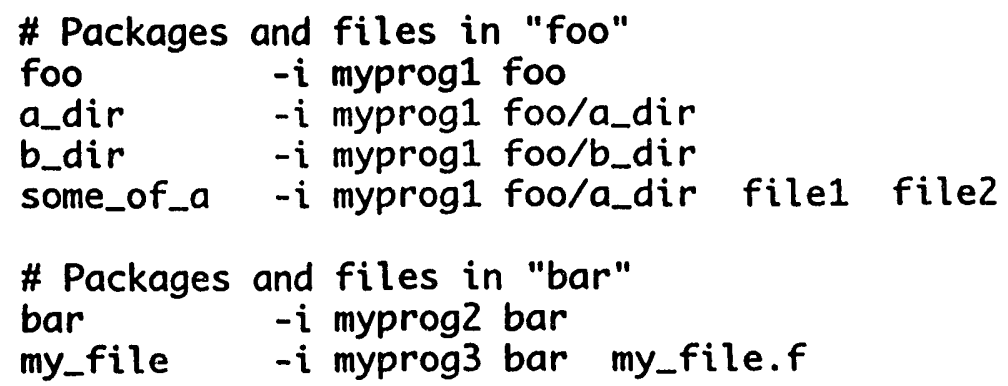

Then myprog2 would run whenever bar or any of its components were committed. However myprog1 and myprog3 would never run. This is because cave cracks the modules database and always calls cvs with the pathname rather than the module name, so that the directory structure remains correct. This means that cvs never knows that the module was specified.

cave can interpret the -a option. It assumes that everything to the right of the -a is either a pathname or a module key. This option allows several different directories to be referred to as a group by one name.

$\begin{array}{ll}\begin{array}{l}\text { b_dir } \\ \text { some_of_a } \\ \text { bar }\end{array} & \begin{array}{l}\text { foo/b_dir } \\ \text { foo/a_dir file1 file2 } \\ \text { bar }\end{array} \\ \text { special } & -a \text { b_dir some_of_a bar }\end{array}$

cave can interpret the "\&" character. Use this if there is a module you want to check out every time another module is checked out. For example:

$\begin{array}{ll}\text { some } & \text { foola_dir file1 \&bar } \\ \text { bar } & \text { bar }\end{array}$

This causes bar to be checked out whenever some is checked out.

There are some restrictions. None of the modules which use an " $\&$ " may point to a module which itself uses an "\&" or a "-a". None of the modules which use a "a" may" point to a module which itself uses a "-a".

If the module key and the actual directory name are the same, use the "-a" option rather than an "\&". If you use the " $\&$ ", some things may get checked out twice and placed in two different locations. For example: 
Good:
a_dir
foo/a_dir
bar
-a bar foo/a_dir

Bad:
a_dir
foo/a_dir
bar
bar \&a_dir

cave assumes that any modules file used by the project is set up so that a request to check out a source repository results in the entire source repository being checked out. It also assumes that the modules file contains the standard CVSROOT keys:
\# CVSROOT support
CVSROOT
$-i$ mkmodules CVSROOT
modules
- $i$ mkmodules CVSROOT modules
loginfo
commitinfo
-i mkmodules CVSROOT loginfo
rcsinfo
- $i$ mkmodules CVSROOT commitinfo
editinfo
- $i$ mkmodules CVSROOT rcsinfo
cvsignore
- $i$ mkmodules CVSROOT editinfo
- $i$ mkmodules CVSROOT cVsignore 


\section{Appendix D: Using Cave-Scenarios}

The following pages show some possible scenarios for using cave in a production environment. Adapt one or more of these to fit your own situation.

\section{Sources and executables on one workstation.}

To make changes to the source files, do the following.

1. Run cave checkout to get private copies of the files you want to change.

2. Edit your private copies of the files.

3. Run cave update or cave status occasionally if you wish to keep up-to-date with any changes to the files in the repository.

4. Use make to compile the executable in your private workspace.

5. Test the executable.

Repeat the preceding steps as needed, until you are ready to make the changes permanent. When you are ready to install the changes, do the following.

6. Run cave lock update to set an update lock.

7. Run cave update to bring the local files up-to-date with any changes to the repository. Resolve any conflicts. You may need repeat steps $2 \ldots 5$ if there have been changes.

8. Run cave commit to put the changed files into the repository.

9. Use make to compile the executable in your private workspace.

10. Use make to install the executable in a public area. The installation procedure may include several checks. For example:

- Use cmsum to make sure no files were changed after they were installed in the repository.

- Use cmvers to compare the value in the tag file in the repository with a file stored in some other location. 
- Use cmvers to determine a version number to tack onto the end of file names, and compare the new file names with those in the public area.

11. Run cave unlock to release the update lock.

\section{Sources on a workstation and executables on another platform.}

To make changes to the source files, do the following.

On the workstation:

1. Run cave checkout to get private copies of the files you want to change.

2. Edit your private copies of the files.

3. Run cave update or cave status occasionally if you wish to keep up-to-date witr. any changes to the files in the repository.

4. Run cave pack when you are ready to collect the files for transport to the remote workstation.

5. Run cmput or ftp to transport the files to the remote workstation.

On the remote workstation:

6. Use make to compile the executable in your private workspace.

7. Test the executable.

Repeat the preceding steps as needed, until you are ready to make the changes permanent. When you are ready to install the changes, do the following.

On the workstation:

8. Run cave lock update .i.lock:command; .i.lock:update; .i.lock:types:update; i. upciate lock; to set an update lock.

9. Run cave update to bring the local files up-to-date with any changes to the repository. Resolve any conflicts. You may need repeat steps 2-7 if there have been changes.

10. Run cave commit to put the changed files into the repository.

11. Run cave pack to collect the files together for transport to the remote workstation.

12. Run cmput or ftp to transport th, files to the remote workstation.

On the remote workstation: 
13. Use make to compile the executable in your private workspace.

14. Use make to install the executable in a public area, such as/usr/local/apps. The installation procedure may include several checks. For example:

- Use cmsum to make sure no files were changed on the remote workstation after they were moved up from the workstation.

- Use cmvers to compare the value in the tag file moved up from the workstation with a file stored on the remote workstation.

- Use cmvers to determine a version number to tack onto the end of file names, and compare the new file names with those in /usr/local/apps.

- Use cmput to send a message file back to the workstation indicating the success or failure of the installation.

On the workstation:

15. Check for any message files you sent back.

16. Run cave unlock to release the update lock. 


\section{Appendix E: What if I want to...?}

What do I do if I want to get rid of the files I checked out earlier, without committing any changes back into the repository?

The simplest way is to delete them the same as you would any other file. A nicer way, if you reference the history file occasionally, is to run cave release. This command checks that no uncommitted changes are present, that you are executing it from inside or immediately above a cvs working directory, and that the repository name is good. It also leaves a record of its execution in the history file, which simply deleting the files will not do.

What do I do if I want to change the name of a file which already exists in the repository?

Check out the file using cave checkout. Rename the file in your local workspace from old name to new name. Then run cave remove old name and cave add new_name. Finally, commit the change with cave commit.

What do I do if I want to change the name of a directory which already exists in the repository?

I would advise not changing it. However, if you think it is necessary, do the following. Make sure nobody has that directory or anysubdirectories or files checked out. Using the regular Unix mv command, change the name of the directory. If you refer to the directory in any of the files in CVSROOT, check those files out and change the name in them. Commit the files back in.

What do I do if I want to put a file which was removed earlier by running cave remove back into a repository?

If the change hasn't been committed yet, run cave add.

A file which is removed from a repository is stored in a directory named Attic, which is located in the same directory as that which originally contained the file. The only way to retrieve a removed file is manually, by using the normal mv command to move the file out of Attic.

What do I do if I want to get all the files as they existed on a particular date? 
Run cave checkout, using the $-\mathbf{D}$ and $-\mathbf{P}$ options.

What if I want to see which of my local files I have changed?

Run cave status. Another possibility is to run "cvs -n update". The "-n" option tells cvs to give a report as if it had run update, but to not really update anything.

What if I want to see what changes I've made to my local files?

Run cave diff. 


\section{Appendix F: Cave Command Summary}

add [-R] [-k kflag] [-m message] [-F list] file ...

checkout [cvs_checkout_opts] [-res] [-F list] [path ...]

commit [-InR] [-m msg |-f file] [-r revision] [-t tag] [-T] [-unres] [-F list] [path ...]

diff [-I] [-q] [-cefhn] [-biwt] [[-r rev1 1-D datel ] [-r rev2 I-D date2 ]] [-F list] [file ...]

export [-fnQq] -r tag I-D date [-F tist] [module ...]

header [-q] [-c chars] [-k keyword [-k keyword ...]] [-F list $]$ file [...]

help [command]

history [-d root_dir] [-report] [-flags] [-options] [-F list] [file...]

lock [-m message] lock_type

$\log [-1][$ rlog-options] [-F list $]$ [file ...]

pack [co_opts] [-c] [-d dir] [-w from] [-T I -Z] [-i distrib] [-F list $][[-\mathbf{x}$ other $] \ldots][$ path ...]

print $[$ co_opts $][-\mathrm{w}$ from $][-1][-\mathrm{c}][-\mathrm{p}$ "lpr_line"] [...] [-F list $][$ path ...]

release $[-\mathrm{dQq}][-\mathrm{F}$ list $]$ module ...

remove $[-\mathbf{R}][-\mathrm{F}$ list $][$ file ...]

reserve $[-\mathrm{m}$ message] $[-\mathrm{F}$ list $]$ path $[\ldots]$

rtag [-aflnQqR] [-b] [-d] [-r tag 1-D date] [-F list] symbolic_tag module ...

status [-IR] [-v] [-F list] [file ...]

$\operatorname{tag}[-\mathbf{I Q q R}][-\mathbf{b}][-\mathbf{d}][-\mathbf{F}$ list $]$ symbolic_tag [file ...]

unlock lock_type I-n lock_name

unreserve $[-\mathrm{F}$ list $]$ path $[\ldots]$

update [-AdfIPpQq] [-j branch] [-r tag I-D date] [-I name [-I name...] [-F list] [file ...] 
verify

version

what [is I are] key [args] 


\section{Glossary}

branch Each revision of an rcs file is considered to be a node in a tree structure. All nodes which have revision numbers containing two fields (e.g. 1.2, 6.3, etc.) are on the trunk or main branch. Occasionally, a developer will want to create a branch off of the trunk, which is generally done using rtag -b or tag -b. From that point on, all commit changes made within these working sources will be automatically added to a branch revision, thereby not perturbing main-line development in any way. The revision numbers on the branch will have four fields. For example, revision 2.3.1.4 is on branch number 1 started at revision 2.3 from the trunk.

cvs This stands for "Concurrent Versions System". It is a freeware program that runs on most UNIX stations. It acts as a front end to the rcs revision control system.

CVS Whenever a cvs controlled source is checked out into a local workspace, it is place in a directory created by cvs. This directory also contains a subdirectory called CVS which contains several files used by cvs for administrative purposes. This subdirectory should not be touched as long as the user plans to return the local files to the source repository.

CVSROOT This is used in two places The first is an environment variable which gives the absolute pathname of the currently active cvs root directory. The second is the name of a subdirectory of the root directory which contains several files used by cvs for administrative purposes.

head The most recent revision of a file on the trunk. Generally, the head revision is the default for most cvs commands.

loginfo file A special file used by cvs for administrative purposes. It can use any sort of filter program, such as cat, to automatically send output to a log file whenever source files are committed. The loginfo file is contained in the directory CVSROOT, located in the root directory.

module A name or key assigned in the modules file to reference subdirectories and files in a source repository. For example, you could define "my.pkg" to mean all the files in the directory "foo/my.pkg". The key may be different than the real name of the subdirectory or file in the source repository.

modules file A special file used by cvs for administrative purposes. It is used define alias's, and to define keys or pointers to the source repository, its subdirectories, and its source files. The modules file is contained in the directory CVSROOT, located in the root directory.

project The complete set of files and directories under cave control which are used to make an executable or collection of executables. This may include source files, object files, make files, scripts, etc. 
project directory The directory in your local workspace where sources checked out by cvs are placed. It is named after the project, as determined from the project description file. The sources are placed so that the directory structure under the project directory echoes that of the root directory.

project description file A project description file is a text file which describes the root directories, source repositories, subdirectories used by a project. It contains the information needed to customize the operation of the cave tool.

repository A repository is a directory inside a root directory. It contains the cvs controlled file(s)

revision A file has a revision number which indicates what branch it is from, and how many times it has been changed. This number is (generally) changed automatically. For example, if a file is assigned an initial revision number of 1.1 , the next time it is checked in the revision will be 1.2. If the revision number has more than two fields (such as 1.2.1.1), it indicates that the version of the file is from a branch, rather than the main trunk.

root directory A root directory is a directory that contains one or more repositories and a CVSROOT directory.

source repository See repository.

symbolic tag See tag.

tag A symbolic name or marker assigned by a user to one or more files in a repository. It can be thought of as a version number for a group of files. The tag must start with a letter, and must not contain a semicolon, colon, dollar sign, at sign, comma or period (;:\$@,.).See also: revision

tail The tail of a pathname is the last file or directory in that pathname. For example, if the pathname is /home/people/me/foo, the tail of the pathname is foo.

trunk See branch. 


\section{Index}

\section{\$Id\$ See header \\ \$Log\$See header \\ access}

controls $111,116,117,122,123$, $126,133,137,139,142$

in project description file $9,65,81$, 82,85 description 83

example $110,112,117,122,125$, 127,130

add $49,62,155$

adding a directory $10,133-137$

adding a file 139-142

description 16-17, 107, 157

history 33

locks 84

new synonym 16

synonyms 16

adding a file or directory See add

administrative directory

CVS 10, 16, 27-28, 38, 42, 47, 49, $129,133,139,143,159$

CVSROOT $21,24,28,34,43,47$, $62,82,92,107,111,112-115$, $117,118-121,123,125,136-137$, 141-142, 145-146, 159

Attic See also remove, 49, 52, 138, 155 cave See also project description file, 10 , 160)

description 15,107

example $11,30,31,32,127-146$, 151-153

execute line 15

help 32

relation to cvs $9,10,11,15,49,61$ version See version command

CAVEMAN

advantages 7

assumptions 9-10

disadvantages 7-8

documentation 11

purpose $1,15,107-108$

requirements 105

restrictions $9-10$

tools $69-79,105$ cave 107

cmget 70

cmlog $24,72-73,113,118$

cmlog_accum $24,74-75,76-77$

cmlog_prep 74-75, 76-77

cmput $71,152,153$

cmsum $78,151,153$

cmvers $79,151,152,153$

underlying programs $1,105,107-108$

CAVE_PD

environment variable $9,15,81,128$, $129,133,139,143$

check in See commit

check out See checkout

checking in a file or directory See commit

checking out a file or directory See checkout, export

checkout See also export, 16, 38, 41-42, $45-46,49,86-87,89,100,155,156$ and export 21,27, 28

co synonym $18,128,134,136,140$, $141,143,145$

description 18-21, 107, 157

example $112,118,125,127-132$, $133,139,143,151,152$

get synonym 18, 128, 134, 140, 143

history 33

locks $20,36,57,84$

out synonym 18

referenced in modules file 27,114 , 120,148

synonyms $18,128,134,136,140$,

ci $141,143,145$

synonym for commit 22

cmaet 70)

cmlog See also log, 24, 72-73, 113, 118 cmlog_accum See also log, 24, 74-75,

$76-77$

cmlog_prep See also log, 74-75, 76-77

cmput 71, 152, 153

cmsum $78,151,153$

cmvers $79,151,152,153$

co

example $128,134,136,140,141$, 143,145

rcs command 19, 25, 27, 30, 37, 41, $45,52,60$

synonym for checkout $18,128,134$, $136,140,141,143,145$

collecting files and directories See pack commit 10, 16, 49, 74, 76, 85, 88, 90,

$93,107,148,155$ 
ci synonym 22

description 22-24, 107, 157

example $115,121,125,137,142$, $146,151,152$

history 33,102

in synonym 22

locks $23,36,57,84$

put synonym 22

referenced in modules file 114,120 , 148

synonyms 22

commitinfo 90

description 101

example 74-75, 76-77

referenced in modules file 9,114 , $120,147,150$

committing a file or directory See

commit

conflict resolution $61-62,107,151,152$

controlling access to a project See access cvs

admin $16,20,42,46$

description 99

rcs synonym 99

synonyms 99

administrative directory $10,16,27$ -

$28,38,42,47,49,129,133,139$. 143,159

behavior 10,148

commands not in cave $99-100$

definition 159

description 1

documentation 1,11

import 28

description 99

rdiff

example $115,121,122,124$

description 100

patch synonym 100)

synonyms 100

support files 101-102

cvsignore 101

editinfo 101

history $34,48,98,101,155$

loginfo $72-73,74-75,76-77,101$, $103,112,118,125,159$

modules $9,18,20,21,22,27,28$, $40,42,43,44,45,46,47,48,52$,

$53,86,93,101,103,112,118$,

$125,128,133,136,137,139$.

$141,142,143,145,146,147$.

150,159

rcsinfo 101

support programs cvsinit 103, 112, 118

mkmodules 103

version 106

cvsignore

cvs support file 101

description $101,114,120-121$

example 114, 120-121

referenced in modules file 9,150

cvsinit

cvs support program 103, 112, 118

description 103

example 111,118

CVSROOT

administrative directory $21,24,28$, $34,43,47,62,82,92,107,111$, $112-115,117,118-121,123,125$, $136-137,141-142,145-146,159$

definition 159

environment variable $73,81,92,98$, $101,103,111,118,123,147$

delete

referenced in modules file 9,150

diff

synonym for remove 49

command 156

description 25-26, 157

locks 36, 57, 84

utility 26,100

diff3 11,106

version 106

differences in files See diff, diff3

directories

in project 65

editinfo

cvs support file 101

description 101

referenced in modules file 9, 114 , $120,147,150$

environment variable

CAVE_PD 9, 15, 81, 128, 129, 133, 139,143

CVSROOT $73,81,92,98,101,103$, $111,118,123,147$

RCSBIN 97

export See also checkout

and checkout 21,28

description 27-29, 157

locks 84

read synonym 27

synonyms 27

extract lock 20, 36, 57, 84

extracting a file or directory See

checkout, export

file differences See diff, diff.3 
files

freeze

in project 65

synonym for tag command 55

gathering files and directories See pack get

synonym for checkout $18,128,134$, 140,143

group

id $111,116,117,122,123,126,133$, $137,139,142$

in project description file $66,81,82$, 83

description 83

example $109,112,117,122,125$, 127,130

header

\$Id\$ 115, 121, 124, 135, 141

\$LOg\$ 115, 121, 124, 135, 141

command

description 30-31, 157

example 30-31

help

example of $\log$ file 111

description 32,157

hi

example 11,32

history

synonym for history command 33

command 98

description 33-35, 157

locks 84

cvs support file $34,48,98,155$

in description 101-102

synonym for commit 22

insert lock 23, 36, 57, 84

lock See also unlock, 15, 66

command 151

description 36,157

extract $36,57,84$

ignoring with $-\mathrm{L} 15$

in project description file $9,66,81$, 82

description 84

example $110,112,117,122,125$, 127,130

insert $36,57,84$

panic $15,36,57,84$

types

extract $36,57,84$

insert $36,57,84$

panic $15,36,57,84$

update $36,57,84,151,152,153$ update $36,57,84,151,152,153$

locking the sources See lock, unlock $\log$

description 37-38, 157

file $22,72-73,74-75,76-77$

example 111

specifying See loginfo

updating See also cmlog,

cmlog accum, cmlog prep

message $22,24,72-73,74-75,76-77$

rlog synonym 37

synonyms 37

loginfo 88

cvs support file 72-73, 74-75, 76-77, $101,103,112,118,125,159$

description 101

example 72-73, 74-75, 76-77, 113, 118-119

referenced in modules file 9,114 ,

login_grp $120,147,150$

lpr

in project description file 82 description 85

print spooler $44,66,91$

mail

synonym for print command 44

in project description file $24,66,82$ description 85

example $110,112,117,122,125$, 127,130

sending log message 72,74

when committing See mail in project description file

when ignoring locks 15

marking a file See rtag, tag

mkmodules

cvs support program 103

description 103

example $9,114,120,147,150$

module

definition 147,159

modules

cvs support file $9,18,20,21,22,27$, $28,40,42,43,44,45,46,47,48$, $52,53,86,93,101,103,112$,

$118,125,128,133,136,137$, $139,141,142,143,145,146$, $147-150,159$

description 9, 101, 147-150

example 9, 113-114, 119-120, 147, 150

referenced in modules file 9,114 , $120,147,150$ 
new

out

$$
\text { synonym for add } 16
$$

pack

synonym for checkout 18

command 10,86, 148, 152

description $39-43,157$

in project description file $39,66,82$ description 86 example $110,112,117,122,125$, 127,130

locks 84

panic lock $15,36,57,84$

patch

perl

synonym for cvs rdiff 100

documentation 11

scripts $9,11,15$

version 106

postcheckout

in project description file 21,82 description 86-87

postcommit

in project description file 24,82

precheckout description 88

in project description file 21,82 description 89

precommit

in project description file 24,82

print description 90

command 91 description 44, 47, 157

in project description file $44,66,82$ description 91

example $110,112,117,122,125$, 127,130

locks 84

lpr synonym 44

synonyms 44

project

definition 9, 159

directory with same name $20,26,28$, $39,48,54,55,61,127,129,160$ restrictions $9-10$

project description file See also verify, what, 15, 22-23, 24, 28, 39, 43, 44, $57,107,127,129$

definition 160

description 9, 81-93

example 109-110, 112, 117-118, $122,125-126,127,130$

information about 65-67 required $17,20,23,26,28,35,36$,

$38,42,46,48,49,51,53,54,56$, $57,59,62$

put

verifying 63

rcs

synonym for commit 22

co $19,25,27,37,41,45,52,60$

cvs as front end 1,106, 107, 159

documentation $11,16,20,42,46,99$

files 34,100

keywords 20, 28, 30-31, 42, 46, 100

location 97

rlog 37-38

synonym for cvs admin 99

version 100, 106

RCSBIN environment variable 97

rcsdiff 25,26

rcsinfo

cvs support file 101

description 101

referenced in modules file 9,114 , $120,147,150$

read

synonym for export 27

rel

synonym for release 48

release 155

description 48, 157

history 33,155

locks 84

rel synonym 48

synonyms 48

releasing a file or directory See release

remove $17,52,143,155$

delete synonym 49

description 49, 107, 157

example 143-146

history 33

rm synonym 49

synonyms 49

removing a file See remove

repository

definition 160

in project description file $66,81,82$ description 92

example $110,112,117,122,125$, $126,127,130$

restrictions $9-10$

res

synonym for reserve command 50

reserve $20,23,66$

command 18,91

description 50-51, 157 

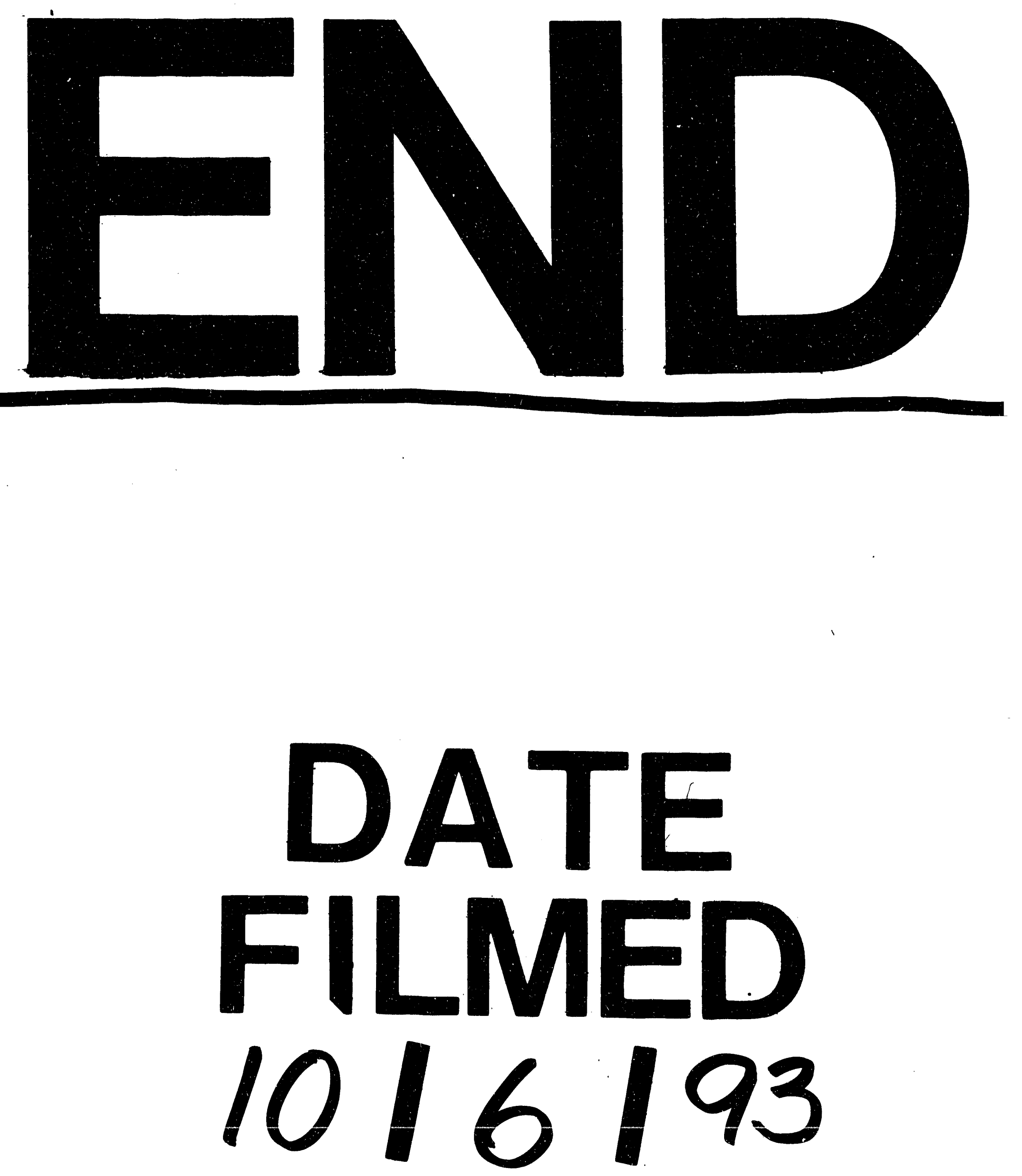


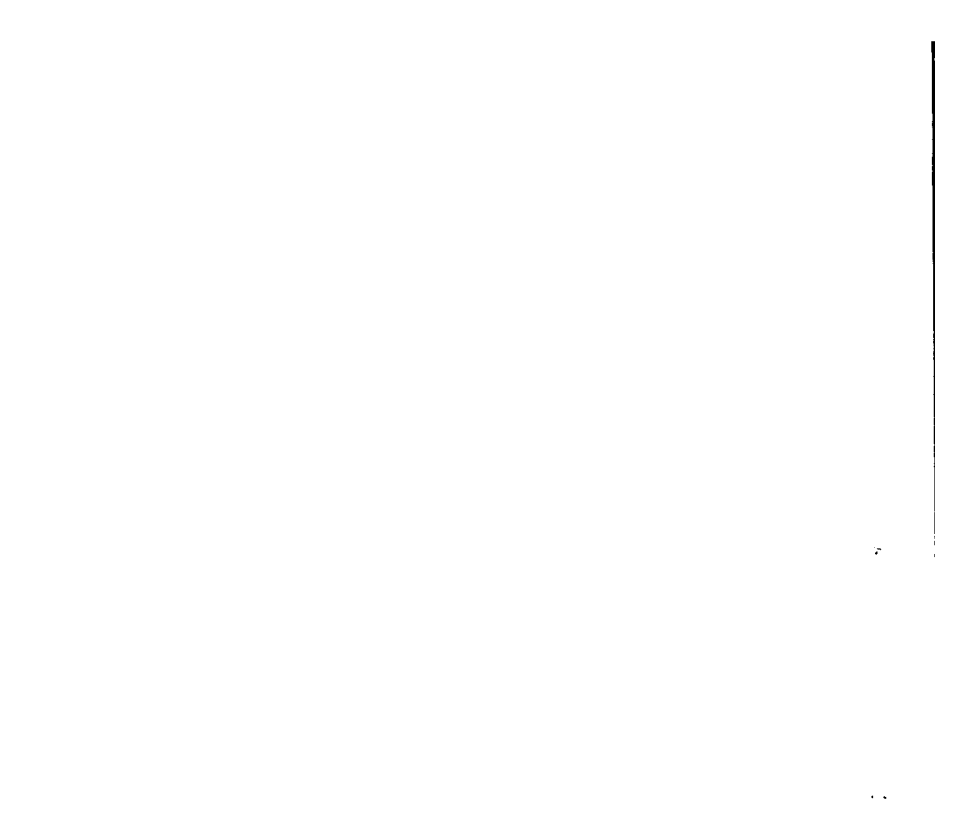

